\title{
THESIS
}

\section{IMPACTS OF ASSIMILATING VERTICAL VELOCITY, LATENT HEATING, OR HYDROMETEOR WATER CONTENTS RETRIEVED FROM A SINGLE REFLECTIVITY \\ DATA SET}

\author{
Submitted by \\ Yoonjin Lee \\ Department of Atmospheric Science
}

\author{
In partial fulfilment of the requirements \\ For the Degree of Master of Science \\ Colorado State University \\ Fort Collins, Colorado
}

Spring 2017

Master's Committee:

Advisor: Christian D. Kummerow

Co-Advisor: Milija Zupanski

Steven C. Reising

Susan C. van den Heever 
Copyright by Yoonjin Lee 2017

All Rights Reserved 


\begin{abstract}
IMPACTS OF ASSIMILATING VERTICAL VELOCITY, LATENT HEATING, OR HYDROMETEOR WATER CONTENTS RETRIEVED FROM A SINGLE REFLECTIVITY DATA SET
\end{abstract}

Assimilation of observation data in cloudy regions has been challenging due to the unknown properties of clouds such as cloud depth, cloud vertical profiles, or cloud drop size distributions. Attempts to assimilate data in cloudy regions generally assume a drop size distribution, but most assimilation systems fail to maintain consistency between models and the observation data, as each has its own set of assumptions. This study tries to retain the consistency between the forecast model and the retrieved data by developing a Bayesian retrieval scheme that uses the forecast model itself for the a-priori database. Through the retrieval algorithm, vertical profiles of three variables related to the development of tropical cyclones, including vertical velocity, latent heating, and hydrometeor water contents are derived from the same reflectivity observation. Vertical velocity and latent heating are variables related to dynamical processes of tropical cyclones, whereas hydrometeors are byproducts of those processes. Each retrieved variable is assimilated in the data assimilation system using a flow dependent forecast error covariance matrix. The simulations are compared to evaluate the respective impact of each variable in the assimilation system.

In this study, the three assimilation experiments were conducted for two hurricane cases captured by the Global Precipitation Measurement (GPM) satellite: Hurricane Pali and Hurricane Jimena. Analyses from these two hurricane cases suggest that assimilating latent heating and 
hydrometeor water contents have similar impacts on the assimilation system while vertical velocity has less of an impact than the other two variables. Using these analyses as an initial condition for the forecast model reveals that the assimilations of retrieved latent heating and hydrometeor water contents were also able to improve the track forecast of Hurricane Jimena. 


\section{ACKNOWLEDGEMENTS}

First and foremost, I would like to express my sincere gratitude to my two advisors for their guidance for my research. Dr. Christian Kummerow broadened my research perspectives greatly in the areas of atmospheric science. Dr. Milija Zupanski greatly helped me understand and run the MLEF model which seemed impossible at first. I appreciate both of my advisors being so patient with me. I would also like to extend my gratitude to Drs. Susan van den Heever and Steven Reising for serving on my committee. A special thanks to Dr. Kummerow's research group and Dr. Zupanski's research group for always leading me to solutions whenever I ran into problems. Also, I would like to thank Dr. Noh of the Cooperative Institute for Research in the Atmosphere (CIRA) for her insights in optical properties of hydrometeors. And last but not least, I thank my family and friends for their unfailing support. 


\section{TABLE OF CONTENTS}

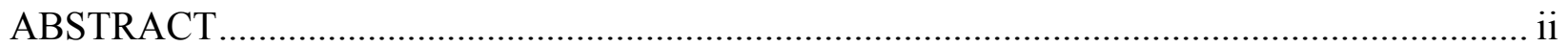

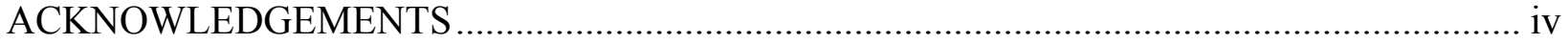

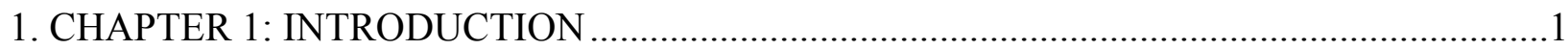

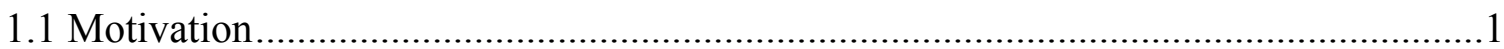

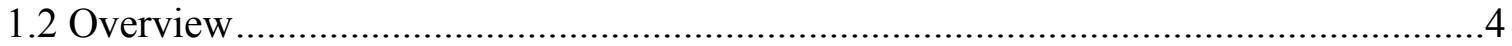

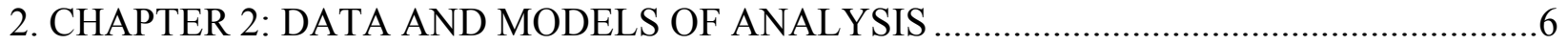

2.1 Global Precipitation Measurement (GPM) …………..............................................

2.2 Weather Research and Forecasting (WRF) .........................................................

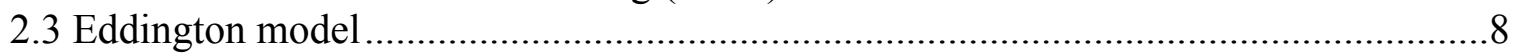

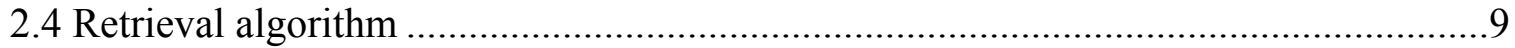

2.5 Maximum Likelihood Ensemble Filter (MLEF)......................................................10

2.6 International Best Track Archive for Climate Stewardship (IBTrACS) .....................13

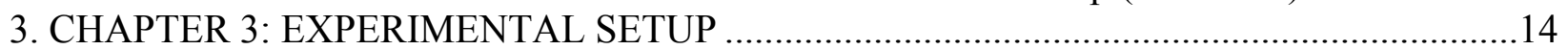

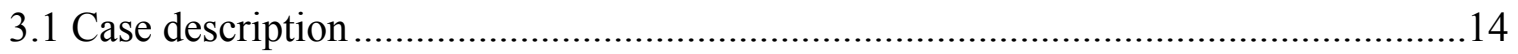

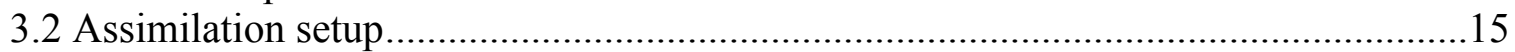

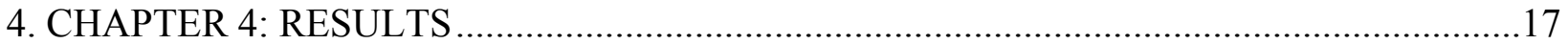

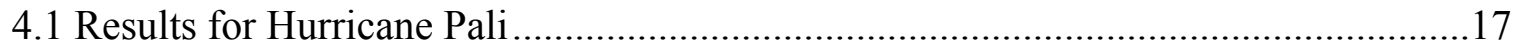

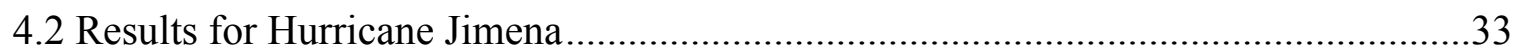

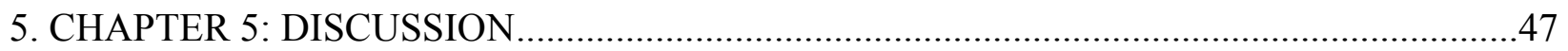

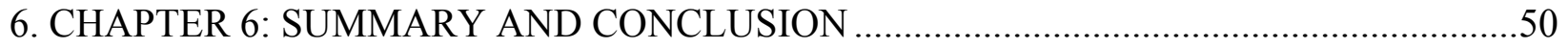

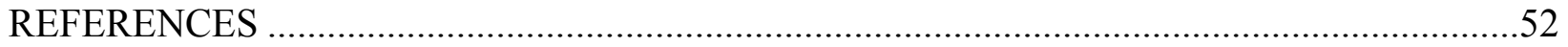

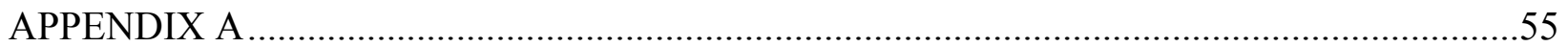




\section{CHAPTER 1: INTRODUCTION}

\subsection{Motivation}

With the advance of numerical weather prediction (NWP) models, specification of the initial conditions has become increasingly more important. Since the prediction made by the model is strongly impacted by the initial conditions, it is clearly important to have the initial conditions properly specified (Kalnay 2003). The most efficient and mathematically consistent way of improving initial conditions for NWP models using observations is data assimilation (DA). DA can be defined as a mathematical method based on Bayes' theorem for optimally blending the information from observations and the model. In practical NWP applications, the optimal analysis in DA is created by minimizing the cost function that measures the mathematical distance from the model and from the observations. Critical for DA success is the specification of forecast and observation error uncertainties. Proper specification has led to remarkable progress in forecasting capability.

One of the most commonly used observation data in DA systems is satellite data due to its high temporal and spatial coverage. Assimilation of satellite data was initially used only in clear-sky regions because optical properties of clouds and their uncertainties are not fully understood and they introduce significant uncertainties. Directly measured quantities, such as temperature or moisture content, were assimilated. Recently, the use of radiances has become more prevalent since fast and accurate Radiative Transfer Models (RTM) were developed and used as an observation operator (Matricardi et al. 2001). This avoids having to specify retrieval errors that were always uncertain, in favor of radiance errors which are well characterized. Assimilating clear-sky radiances in the National Centers for Environmental Prediction (NCEP) 
operational global analysis-forecast system led to a dramatic improvement in forecasting mass and wind fields (Caplan et al. 1997; Derber and Wu 1998). European Centre for Medium-Range Weather Forecasts (ECMWF) also changed from assimilating retrievals to radiances around the same time and demonstrated success with the Television Infrared Observation Satellite (TIROS) Vertical Sounder (TOVS) clear-sky radiance data using variational data assimilation (Andersson et al. 1994). Assimilation of radiances has also been used in tropical cyclone (TC) applications, but considerable research is still being performed using retrieved products (Liu et al. 2010). Xu et al. (2013) showed some improvements in forecasting two TCs by assimilating clear-sky radiances from the Infrared Atmospheric Sounding Interferometer (IASI), but pointed out the importance of cloud detection schemes. The choice of a cloud detection scheme is critical in clear-sky data assimilation because too stringent a control discards too much data, while too lenient a control can include cloud contaminated pixels. Although clear-sky information could be useful in ameliorating environmental temperature and moisture profiles in the vicinity of TCs, it is not an effective way to use the vast amount of available data around the TC core region where there are few clear regions. Furthermore, clear sky assimilation schemes can make little or no use of dedicated satellites such as the Tropical Rainfall Measuring Mission (TRMM) and its successor, the Global Precipitation Measurement (GPM). The DA community is therefore slowly moving towards cloudy-sky DA, even though it is far more complex than clear-sky DA.

Cloudy scenes are more difficult to assimilate because (a) clouds are not continuous variables (unknown correlation in time and space) and (b) the forecast models often do not explicitly diagnose all of the geophysical parameters needed by the observation operator (e.g. ice particle shapes and density or number of drops). Despite the difficulties, much effort has been dedicated to assimilate cloud affected data. Results thus far appear comparable to clear-sky 
radiance assimilation. Zhang et al. (2013) assimilated cloud affected Advanced Microwave Sounding Unit-A (AMSU-A) radiances in NOAA's operational Hurricane Weather Research and Forecasting (HWRF) system and showed that the cloudy-sky radiance assimilation outperformed clear-sky radiance assimilation. Another useful observation in cloudy-sky assimilation is radar reflectivity. Both ground-based radar and space-borne radar can bring useful information to the DA system. Okamoto et al. (2016) recently published a paper using both radiance and reflectivity data from GPM. Their results showed that the combined assimilation of radiances from GPM Microwave Imager (GMI) and reflectivities from the Dual-frequency Precipitation Radar (DPR) had the most positive impact but that GMI alone had more impact than DPR. Greater impact from GMI was mostly attributed to two factors. One of them was a wider swath of GMI yielding more observation data, and the other one was a limited sensitivity of DPR only to precipitating hydrometeors, although DPR has vertically distributed information.

In addition to radiance and reflectivity, retrieved products such as hydrometeor water contents or mass-weighted diameter $\left(\mathrm{D}_{\mathrm{m}}\right)$ of the drop size distribution (DSD) can also be used as an observation. However, derived products contain uncertainties coming from the DSD retrieval itself. DSDs of cloud hydrometeors have been examined by numerous studies but there is a fundamental difficulty in defining a unique probability distribution due to the complexity of observed DSD. This can lead to inconsistencies between the DSD in the forecast model, the RTM, and the retrieval algorithm. This inconsistency can accumulate significant amounts of uncertainty because observed quantities can be very sensitive to DSDs. (See Appendix A.) For instance, the Weather Research and Forecasting (WRF) model has 22 microphysical schemes with different DSDs. The Community RTM (CRTM), which is one of the most widely used RTMs, has a lookup table of cloud optical properties based on a modified gamma distribution, 
(Petty and Huang 2011) and the DPR retrieval algorithm for GPM uses a gamma size

distribution with a fixed shape parameter $(\mu)$. These inconsistencies must be dealt with in a practical manner for DA systems to be successful.

Along with a proper DA method, the choice of variable to assimilate is also a crucial factor since many variables can be derived from observations in cloudy regions. Variables such as hydrometeor water content or drop size distribution parameters can have a direct impact on the model output, while variables related to dynamical processes, such as latent heating or vertical velocity, can directly constrain the motions. Latent heating is related to a phase change of hydrometeors, and vertical velocity is related to convection or subsidence, which are, in turn, related to the production rate of hydrometeors. Some models have started to assimilate these indirect variables. The NCEP High-Resolution Rapid Refresh (HRRR) model is one of those models and it assimilates latent heating rates derived from an observed radar reflectivity (Benjamin et al. 2016). Given these options, and an ability to retrieve hydrometeors as well as latent heating and vertical velocity, this study will explore the impact of assimilating each of three different variables derived from the same radar reflectivity observations in order to assess their respective impact on the DA system.

\subsection{Overview}

Three variables, including one variable with a direct impact on precipitation (five hydrometeor water contents including cloud liquid water, rain, cloud ice, snow, and graupel) and two dynamical variables (vertical velocity and latent heating), will be assimilated in each DA experiments and compared between experiments to determine the relative impact of each variable on the DA system. In order to maintain consistency between the forecast model and the 
retrieved variables, variables are not retrieved from the actual measurements but rather retrieved using a Bayesian scheme that uses the forecast model (from a set of prior TC simulations) for its a-priori database. The database consists of coupled profiles of reflectivity, vertical velocity, latent heating, and hydrometeor water contents. From this database, the reflectivity profile that best matches the observed reflectivity profile is selected, and profiles of the other three variables that are paired with the reflectivity profile are taken as retrieved products. The retrieved products are thus kept consistent with the microphysical scheme in the forecast model. 


\section{CHAPTER 2: DATA AND RETRIEVAL ALGORITHM}

The data and the retrieval algorithm used in the DA experiments are described in this section. Although the three DA experiments assimilate different variables, assimilated data are derived from only one observation from the GPM satellite. With the same radar reflectivity profile from the DPR on GPM, vertical profiles of vertical velocity (VV) (m/s), latent heating (LH) $(\mathrm{K} / \mathrm{s})$, and hydrometeor water contents $(\mathrm{HYDRO})(\mathrm{kg} / \mathrm{kg})$ were retrieved and used in each experiment. This section describes the satellite data and retrieval algorithm with some detail. After the retrievals, each profile was assimilated in the Maximum Likelihood Ensemble Filter (MLEF) model using a flow dependent forecast error covariance matrix. Results from DA simulations were then validated with independent observations, including GMI brightness temperature $\left(\mathrm{T}_{\mathrm{b}}\right)$, DPR reflectivity, and hurricane best track from the International Best Track Archive for Climate Stewardship (IBTrACS).

\subsection{Global Precipitation Measurement (GPM)}

GPM is non sun-synchronous satellite launched on February 27, 2014 as a successor to the Tropical Rainfall Measuring Mission (TRMM). The GPM orbit was designed to encompass a broader coverage $\left(68^{\circ} \mathrm{N}-68^{\circ} \mathrm{S}\right)$ and a wider range of precipitation intensity (Hou et al. 2014). GPM carries the GMI and the DPR. Both instruments are sensitive to a broad range of precipitating hydrometeors. GMI is a conically scanning passive microwave radiometer with 13 channels including $10.65,18.7,23.8,36.5,89.0,166,183.31 \pm 3$, and $183 \pm 7 \mathrm{GHz}$. Each channel has characteristic sensitivity to different types and properties of hydrometeors as explained in Appendix A. DPR measures the three-dimensional structure of precipitation with a Ka-band 
(35.5GHz) precipitation radar (KaPR) and a Ku-band (13.6GHz) precipitation radar (KuPR).

$\mathrm{KaPR}$ and KuPR both have $5 \mathrm{~km}$ footprints but KuPR has a larger cross-track swath of $245 \mathrm{~km}$.

Products from the KuPR and GMI instruments were used in this study. Level 1

reflectivity products from DPR KuPR (version 4) were used to retrieve quantities used in the assimilation as well as for assessment of the assimilated fields, while GMI brightness

temperature from Level 1C (version 4) products were used as an independent test for the three DA experiments.

2.2 Weather Research and Forecasting (WRF)

WRF is used as a background or forecast model in this study. WRF is a widely used weather forecasting model with two options for its dynamical core: ARW (Advanced Research WRF model) and NMM (Non-hydrostatic Mesoscale Model) (Skamarock et al. 2008). Here, WRF-ARW version 3.7 was used with $1^{\circ}$ GFS analysis as an initial condition at the cold start and as a boundary condition throughout the analysis and forecast. Physics that are used in WRF are summarized in the table 2. Among twenty-two microphysical schemes in WRF, the WRF Double-Moment 6 class microphysical (WDM6) scheme (Lim and Hong 2010) was applied but graupel was assigned as the fifth hydrometeor instead of hail. The WDM6 scheme was modified to produce an additional output of vertical profile of latent heating coming from a phase change between hydrometeors as this is not a general output in WRF. In the modified WDM6 scheme, latent heating is calculated by dividing a temperature change from any phase change by the appropriate time step. It has a unit of $\mathrm{K} / \mathrm{s}$. 
Table 1 Table for WRF physics

\begin{tabular}{|c|c|}
\hline Microphysics & Modified WDM6 scheme \\
\hline Long wave radiation physics & RRTM scheme \\
\hline Short wave radiation physics & MM5 shortwave scheme \\
\hline Cumulus parameterization & New Kain-Fritsch scheme \\
\hline Land surface model & Noah land surface model \\
\hline Surface layer & Monin-Obukhov similarity theory \\
\hline Planetary boundary layer & YSU PBL scheme \\
\hline
\end{tabular}

WRF was used both for building the a-priori database for subsequent retrievals, and the DA experiments. Different horizontal resolutions of the WRF model were used for retrieval and DA purposes to allow model errors to be present. Although different horizontal resolutions and number of grids are used, the sigma coordinate in the vertical was set the same so as not to require vertical interpolation in the DA simulation. For the a-priori database used in the retrieval, the WRF model was run with two domains of $300 \times 300 \times 30$ grid points. The horizontal resolution is set to $9 \mathrm{~km}$ for the outer domain and $3 \mathrm{~km}$ for the inner domain. For the DA experiments, on the other hand, a coarser horizontal resolution ( $27 \mathrm{~km}$ for outer domain and $9 \mathrm{~km}$ for inner domain) was set with fewer grid points $(150 \times 150 \times 30)$.

\subsection{Eddington model}

The Eddington model was developed to provide fast, yet accurate microwave brightness temperature and reflectivity estimation (Kummerow 1993). The two stream Eddington approximation expands the radiance and scattering phase function in a series of Legendre coefficients to first order in the cosine of zenith angle. The basic radiative transfer is calculated with discrete ordinate solutions. Since it was created primarily for microwave frequencies, the Eddington model focuses on both absorption and emission, as well as multiple scattering in a plane parallel medium. Calculations of radiance and reflectivity requires profiles of temperature, 
pressure, water vapor, surface wind velocity, and hydrometeor water contents, and size distribution as inputs. These are all provided by the WRF model. The radiance calculation requires an additional variable which is the surface emissivity. The Eddington model has its own emissivity model but it was replaced by the Fast Microwave Ocean Emissivity version 5 (FASTEM-5) model (Bormann et al. 2012) in this study. The DSD in the Eddington model was also replaced. DSD plays a critical role in calculating total values of cloud optical properties because it determines the number of drops of a specific drop size which is multiplied by the optical property of each size of drop. The Eddington model has an exponential distribution as a default for the DSD but this is inconsistent with the WRF outputs. Therefore, the WDM6 scheme was implemented in Eddington's Mie calculation instead of its exponential distribution. The optical properties such as absorption efficiency, asymmetry factor, single scattering albedo, and backscatter phase function were calculated assuming spherical drop and they enter the main Eddington module to produce brightness temperature or reflectivity.

\subsection{Retrieval algorithm}

The retrieval algorithm consists of two parts: building an a-priori database and choosing a profile from the database. The database was created from ten WRF simulations. Ten TCs in the Atlantic basin with different intensities varying from category one to four [Gonzalo (2014), Omar (2008), Edouard (2014), Gustav (2008), Cristobal (2014), Arthur (2014), Igor (2010), Katia (2011), Bill (2009), Danielle (2010)] were simulated in WRF-ARW for twelve hours. The Eddington model reads in the appropriate variables from WRF and calculates the reflectivity at each layer. Among calculated vertical profiles of reflectivity, only profiles that contain at least one level of reflectivity exceeding DPR's threshold of $12 \mathrm{dBZ}$ were saved in the database with 
vertical velocity, latent heating, and hydrometeor water contents profiles from the WRF outputs. In total, over two hundred thousand profiles were obtained from the above ten TC simulations.

Once the database is created, the observed reflectivity profiles at each pixel are compared with the simulated reflectivity profiles in the database. The squared difference between observed and modeled reflectivity at interpolated heights is summed for 30 layers (observed reflectivity has much finer vertical resolution) and profiles whose reflectivity profile has the least squared difference are chosen as "retrieved" fields (VV, LH, and HYDRO) for that grid. In order to smooth these values, they are averaged between six neighboring pixels with a rough Gaussian weight of 0.1 for each neighboring point and 0.4 for the center of the grid.

\subsection{Maximum Likelihood Ensemble Filter (MLEF)}

MLEF is an ensemble-based DA method that also includes components of variational data assimilation. Details of MLEF can be found in Zupanski (2005) but it is reviewed here for completeness. As any other DA algorithm, MLEF goes through two steps in the assimilation process: forecast and analysis. In the forecast step, the analysis from the previous time $\left(x_{a}^{n-1}\right)$ is evolved by the prediction model to the current analysis time (n) to obtain the forecast guess used in data assimilation $\left(x_{f}^{n}\right)$. In addition, the ensemble forecast is used to evolve the analysis uncertainty from the previous analysis time to the current time to produce the forecast uncertainty used in DA. The initial conditions for ensemble forecasts are obtained by adding each column of the square-root analysis error covariance $\left(\mathbf{P}_{a}^{1 / 2}=\left(p_{a, 1}^{n-1} \cdots p_{a, N}^{n-1}\right)\right)$ to the analysis vector, followed by a forecast to the next analysis time, as shown below 


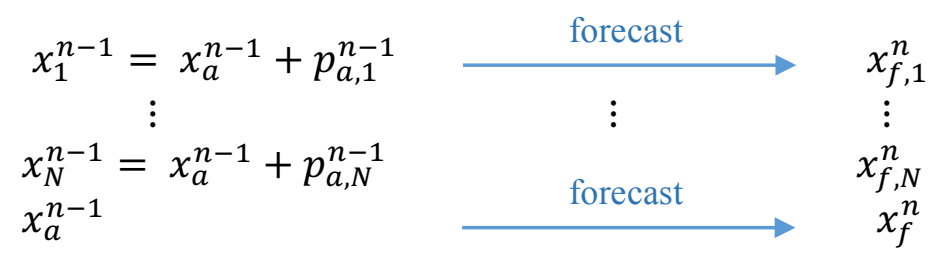

where $\mathrm{N}$ is the number of ensembles and $p_{a, j}^{n-1}$ is the $\mathrm{j}$-th column vector of the square-root analysis error covariance. Finally, the square-root forecast error covariance matrix $\left(\mathbf{P}_{f}^{1 / 2}\right)$ is calculated by subtracting each member of the ensemble forecast from the forecast started from the analysis,

$$
\mathbf{P}_{f}^{1 / 2}=\left(\begin{array}{lll}
p_{f, 1}^{n} \cdots & p_{f, N}^{n}
\end{array}\right) \quad \text { where } \begin{gathered}
p_{f, 1}^{n}=x_{f, 1}^{n}-x_{f}^{n} \\
\vdots \\
p_{f, N}^{n}=x_{f, N}^{n}-x_{f}^{n}
\end{gathered}
$$

The analysis is obtained by maximizing the posterior conditional probability density, in practice achieved via iterative minimization of the cost function. Using common Gaussian error assumption, the cost function is

$$
J(x)=\frac{1}{2}\left(x-x_{f}^{n}\right)^{T} \mathbf{P}_{f}^{-1}\left(x-x_{f}^{n}\right)+\frac{1}{2}[y-H(x)]^{T} \mathbf{R}^{-1}[y-H(x)]
$$

where the superscript $\mathrm{T}$ denotes transpose, $\mathrm{x}$ is the model state vector, $x_{f}^{n}$ is the prior (background) state, and $\mathrm{y}$ is the measurement vector. $H$ represents a nonlinear observation operator that maps variables from model space to observation space and $\mathbf{R}$ is an observation error covariance matrix. $\mathbf{P}_{f}$ is a forecast error covariance matrix defined in ensemble subspace. Before performing actual iterative minimization, the MLEF includes a change of variable

$$
x-x_{f}^{n}=\mathbf{P}_{f}^{1 / 2}\left[I+Z^{T}\left(x_{f}^{n}\right) Z\left(x_{f}^{n}\right)\right]^{-1 / 2} \zeta
$$

where the $\mathrm{j}$-th column of matrix $\mathrm{Z}$ is 


$$
z_{j}=\mathbf{R}^{-1 / 2}\left[H\left(x_{f}^{n}+p_{f, j}^{n}\right)-H\left(x_{f}^{n}\right)\right]
$$

and $\zeta$ is new control variable.

After calculating the optimal control variable from iterative minimization, denoted $\zeta_{\text {opt }}$, the analysis is

$$
x_{a}^{n}=x_{f}^{n}+\mathbf{P}_{f}^{1 / 2}\left[I+Z^{T}\left(x_{f}^{n}\right) Z\left(x_{f}^{n}\right)\right]^{-1 / 2} \zeta_{o p t}
$$

The analysis uncertainty is then calculated in terms of the square-root analysis error covariance $\mathbf{P}_{a}^{1 / 2}$

$$
\mathbf{P}_{a}^{1 / 2}=\mathbf{P}_{f}^{1 / 2}\left[I+Z^{T}\left(x_{a}^{n}\right) Z\left(x_{a}^{n}\right)\right]^{-1 / 2}
$$

where the matrix $\mathrm{Z}$ is now calculated at the minimum.

At the beginning of the DA experiment, however, the analysis and analysis error covariance do not exist, and a procedure to provide the initial state and initial uncertainty is required. In MLEF, the initial uncertainty is calculated using $\mathrm{N}$ time-lagged outputs from a single deterministic forecast centered at the initial time. The time-lagged outputs (Figure 1) are interpreted as ensemble members, and the initial square-root analysis error covariance $\mathbf{P}_{0}^{1 / 2}$ is calculated by subtracting each time-lagged forecast from the central forecast.

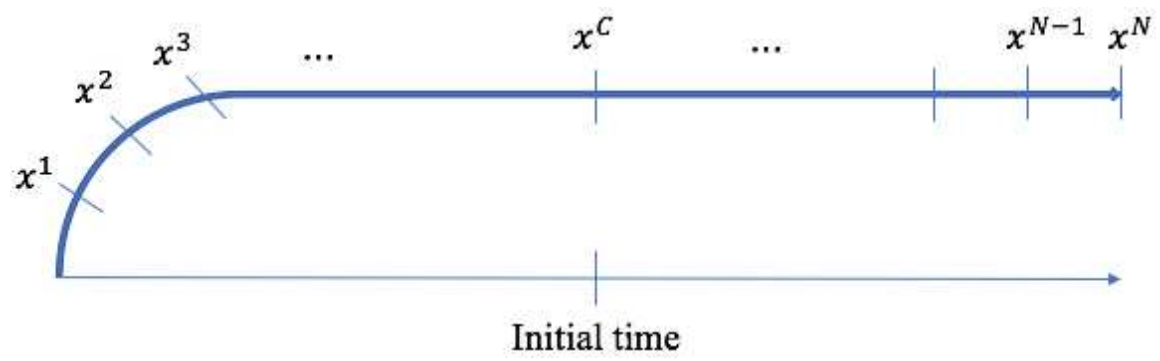

Figure $1 \mathrm{~N}$ number of forecasts conducted to create the analysis error covariance at the initial time

$$
\mathbf{P}_{0}^{1 / 2}=\frac{1}{\sqrt{N}}\left(\begin{array}{llll}
p_{1}^{0} & \cdots & p_{N}^{0}
\end{array}\right) \quad \text { where } \quad \begin{aligned}
p_{1}^{0}=x^{1}-x^{c} \\
\vdots \\
p_{N}^{0}=x^{N}-x^{c}
\end{aligned}
$$


The factor $\frac{1}{\sqrt{N}}$ is used to normalize the forecast difference. The initial state can be obtained by interpolating from an analysis or forecast that is produced by another modeling system, or by using the central forecast $x^{c}$.

2.6 International Best Track Archive for Climate Stewardship (IBTrACS)

Hurricane best track data were obtained from IBTrACS. IBTrACS is a global dataset created at NOAA to inventory reported TCs worldwide and their characteristics such as location, wind speed, and time (Knapp et al. 2010). IBTrACS gathers TC information from several agencies and a best track data is stored according to the agency and the basin. Best track data for Hurricane Jimena was available from two sources: The Automated Tropical Cyclone Forecast (ATCF), and the National Hurricane Center (NHC) HURDAT2 Best Track Database in East Pacific basins. Both sources had the same track. ATCF was used for convenience. 


\section{CHAPTER 3: EXPERIMENTAL SETUP}

\subsection{Case description}

Two hurricanes that developed in the Pacific basin were chosen as a proof of concept study. They are Hurricane Pali and Jimena, and both hurricanes were captured by GPM. Pali became a tropical depression on January $7^{\text {th }}$, developed into hurricane on January $11^{\text {th }}$, and reached a category 2 hurricane on January $12^{\text {th }}$. Pali formed much further south $\left(3.4^{\circ}\right.$ North $)$ than is usual for Pacific Hurricanes. Hurricane Jimena developed in the central Pacific basin and deepened to a category 4 hurricane reaching its peak wind speed of $135 \mathrm{kt}$, which is just below category 5 status. Their vertical structures captured by the GPM and 5 day track starting from the assimilation starting date are shown in Figure 2 and 3.
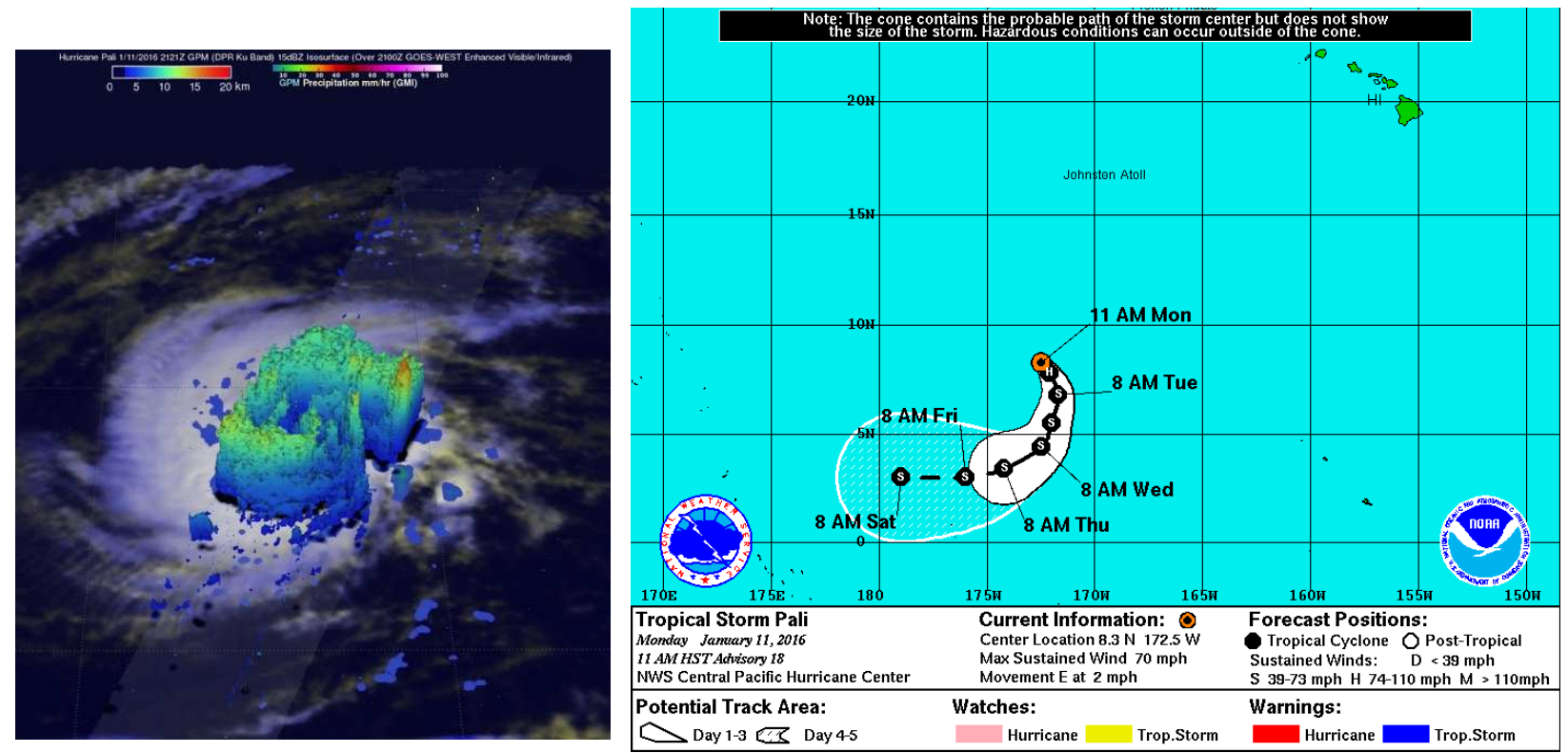

Figure 2 Satellite image of Hurricane Pali by GPM (by Hal Pierce) and its 5 day track (Source: NOAA) 

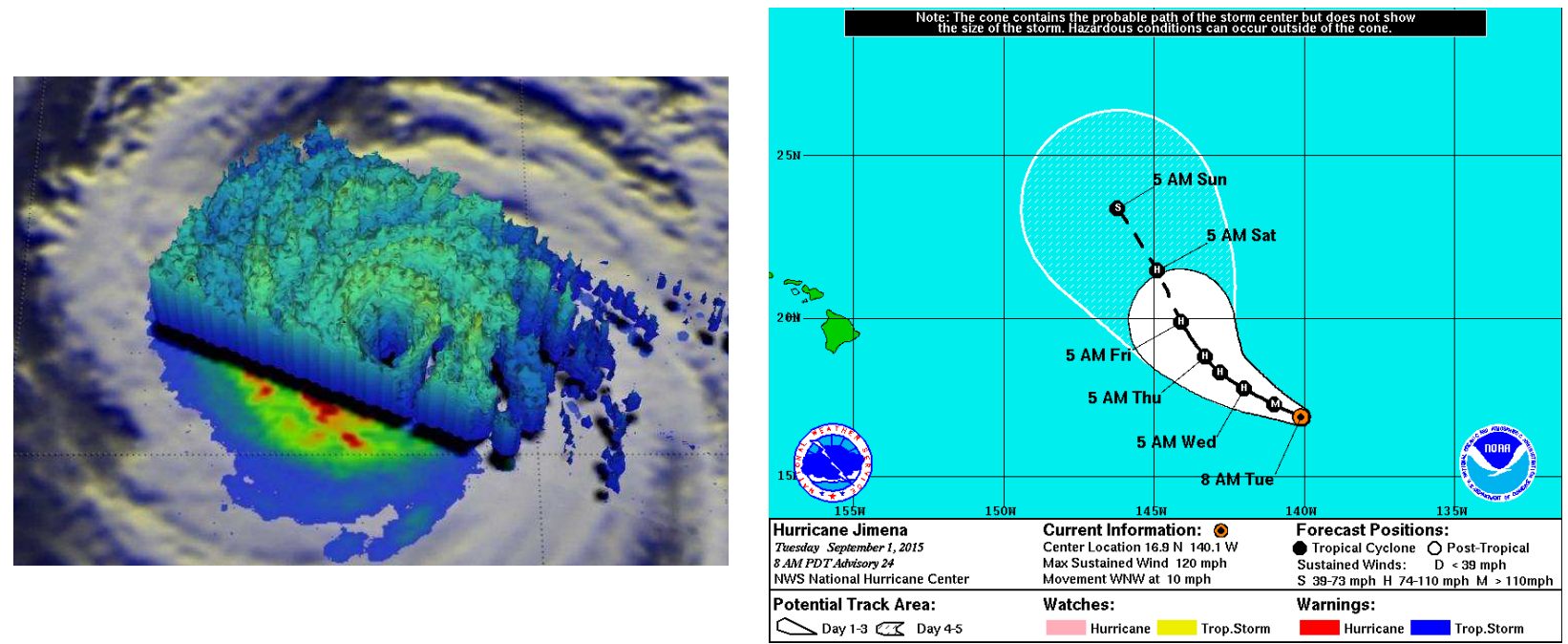

Figure 3 Satellite image of Hurricane Jimena by GPM (by Hal Pierce) and its 5 day track (Source: NOAA)

\subsection{Assimilation setup}

MLEF was compiled with the same WRF model described in section 2.2 and run with 32 ensemble members. Control variables include the general state variables (temperature, perturbation geopotential, horizontal velocities, and perturbation dry air mass in column) and variables that will be assimilated (vertical velocity, latent heating, and five hydrometeor water contents). The observation operator is just a spatial interpolation for all three variables because they are set as control variables in the system.

Quality control (QC) of observational data for each parameter is slightly different. For LH and HYDRO, observations are used if the absolute values of both observed and modeled data are bigger than the threshold of $10^{-6}(\mathrm{~K} / \mathrm{s}$ for $\mathrm{LH}$ and $\mathrm{kg} / \mathrm{kg}$ for HYDRO) to make the distribution of the innovation vector Gaussian, as is assumed in MLEF. Without this process the distribution becomes more like a leptokurtic curve that peaks around 0 because the system incorporates 
unnecessary data such as rain water content above the freezing level or ice hydrometeor content below the freezing level. Vertical velocity does not require this procedure because it has nonzero values most of the time. Instead, because of its vertically staggered grid, it requires an additional treatment. MLEF uses a system that assimilates observation data at non-staggered grids where most control variables reside. Thus, vertical velocity either has to be interpolated into non-staggered grid or a way to process vertical velocity at the staggered grid must be implemented. In order to avoid the vertical interpolation that might bring in more noise, the top grid of vertical velocity is discarded and the remaining observation values are assumed to be the values at the upper non-staggered grid.

Observation errors were set so that the DA system incorporates the observation in the region where the difference between observed and modeled background data is moderate. Observation error whose square value forms a diagonal component of $\mathbf{R}$ is set as a constant of $0.3 \mathrm{~m} / \mathrm{s}, 0.001 \mathrm{~K} / \mathrm{s}$, and $0.0001 \mathrm{~kg} / \mathrm{kg}$, for each $\mathrm{VV}, \mathrm{LH}$, and HYDRO respectively and observation data passes the QC check if $\frac{|y-H(x)|}{\sqrt{R}}<3$. This threshold effectively rejects the observations that are too far from the model. 


\section{CHAPTER 4: RESULTS}

\subsection{Results for Hurricane Pali}

Cloudy scene in Hurricane Pali produced 92940 observation pixels for LH and HYDRO and 96038 points for VV (92940 points after passing the QC that discards data at the top level). These data cover regions from the convective core in the hurricane to rainbands around the core. Figure 4 and 5 show the reflectivity of the best matches from the a-priori database and the observed reflectivity from DPR at two different vertical levels. The lower level is at $1 \mathrm{~km}$ and it describes the liquid precipitation in the scene while the higher level, at $7 \mathrm{~km}$, is above the freezing level and represents ice hydrometeors. The retrieved reflectivity captures the raining scene well enough while reflectivity above the freezing level is not as representative as the lower level.
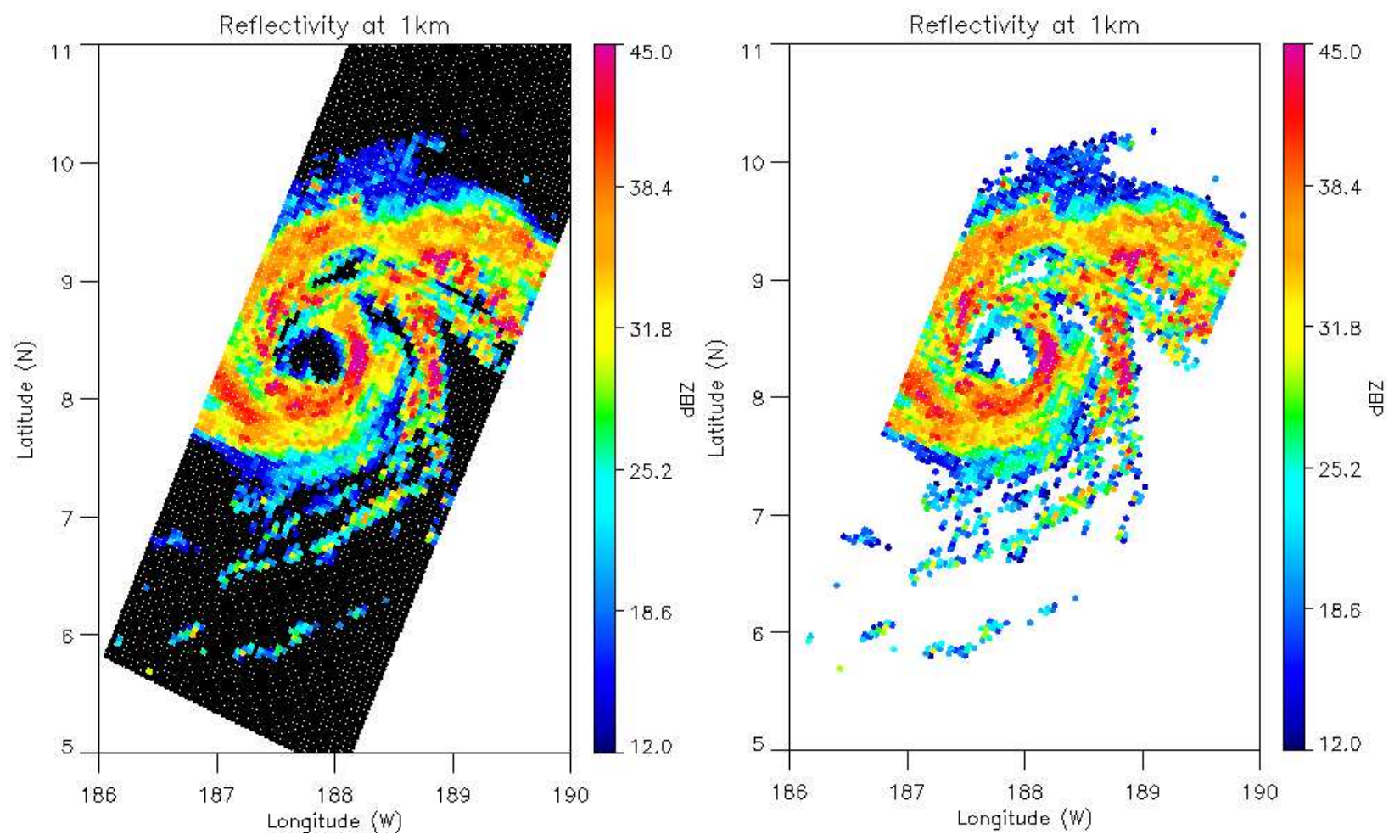

Figure 4 Observed (left) and the retrieved (right) reflectivity horizontal cross-section at $1 \mathrm{~km}$ for Hurricane Pali 

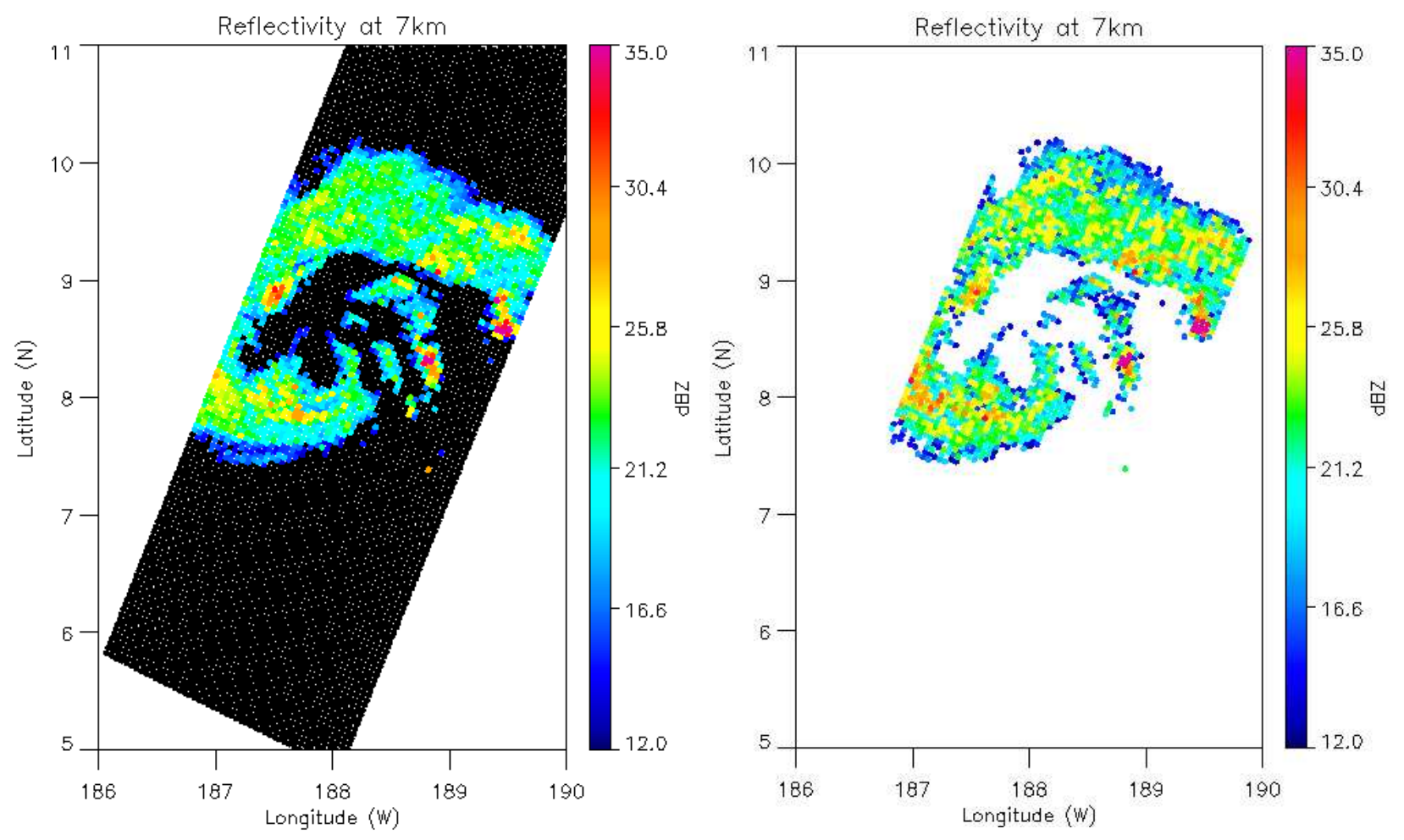

Figure 5 Observed (left) and retrieved (right) reflectivity horizontal cross-section at $7 \mathrm{~km}$ for Hurricane Pali

The reflectivity from the best match is overestimated at $7 \mathrm{~km}$. This might be due to an overestimation of ice hydrometeors from the database, a well-known problem in the forecast models (Gallus and Pfeifer 2008; Han et al. 2013). Horizontal maps of the retrieved fields of the three variables are compared with the background model data next. In Figure 6, VV from the background at $1 \mathrm{~km}$ shows clear updraft and downdraft around the rainbands. The retrieved VV has less structured features but still shows bands of updrafts and downdraft. This might be due to the high variability of VV at the surface. The retrieved VV may be more reasonable than the background because it matches the updraft region with high observed reflectivity especially at $7 \mathrm{~km}$. 

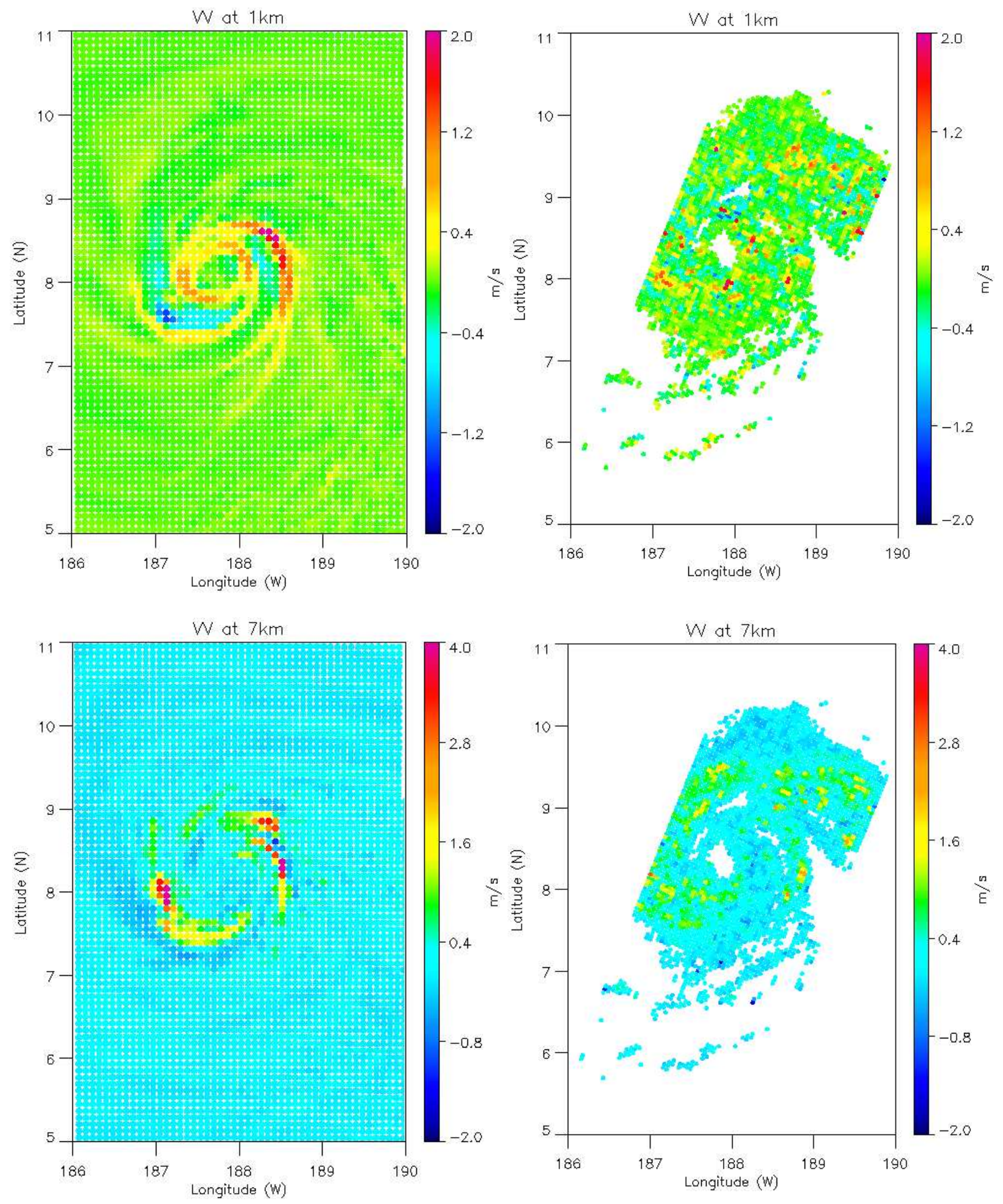

Figure 6 Background(left) and retrieved (right) vertical velocity horizontal cross-section at $1 \mathrm{~km}$ (upper) and $7 \mathrm{~km}$ (lower) for Hurricane Pali 

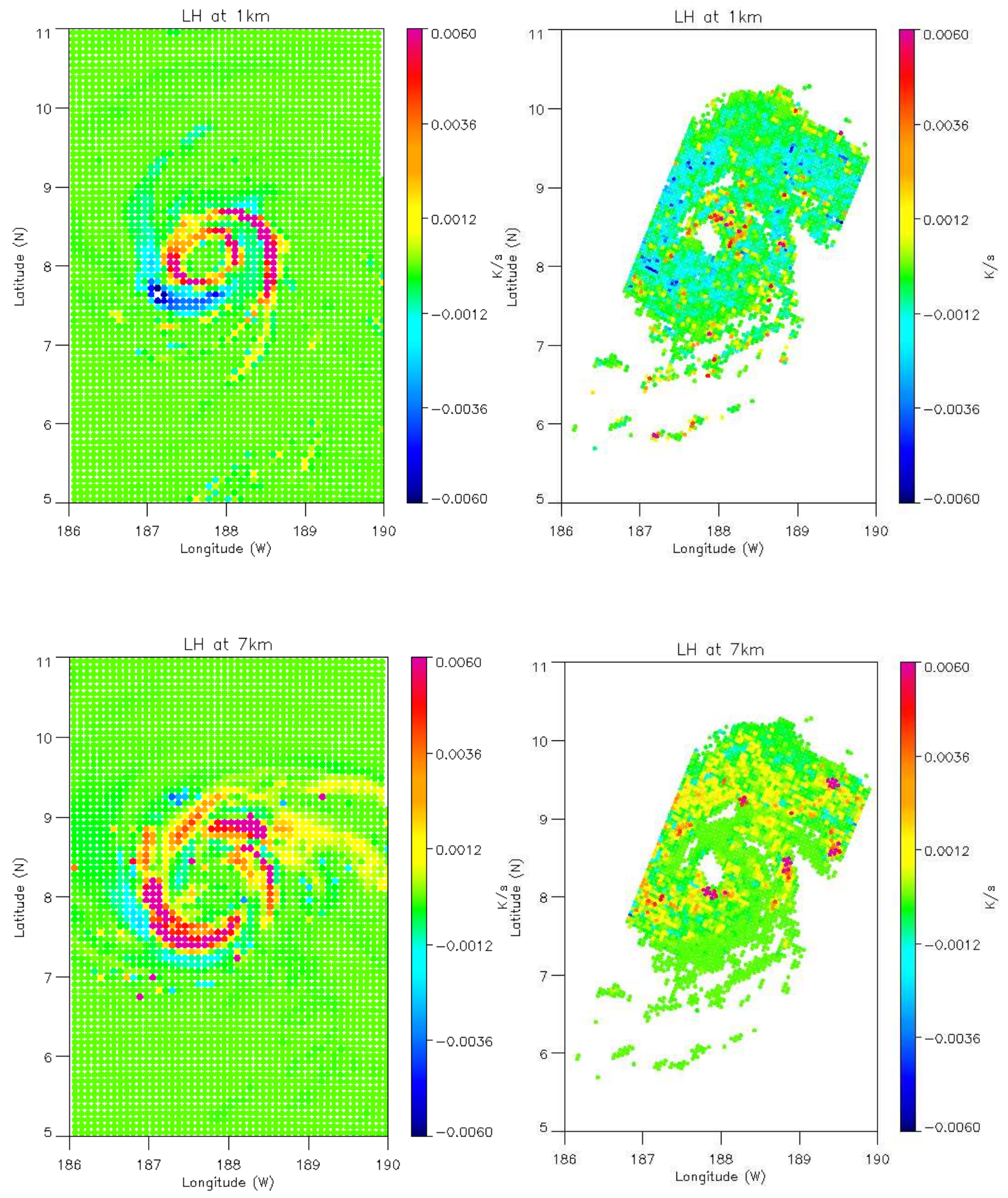

Figure 7 Background (left) and retrieved (right) latent heating horizontal cross-section at $1 \mathrm{~km}$ (upper) and $7 \mathrm{~km}$ (lower) for Hurricane Pali 
In Figure 7, the background LH shows convective (positive LH at the cloud base that slowly decreases with height in the rainbands) and stratiform (negative LH at the cloud base due to evaporation and positive at upper level) features between rainbands. On the other hand, the retrieved LH shows stratiform feature in most of the rainband region with only minimal convective features around the core. In the general, rainbands in TC systems produce rain by a convective motion at the low levels, but the retrieved field does not show any significant amounts of latent heat release by an updraft even in the regions where the updraft is significant. There are few spots where latent heating has a positive value at $1 \mathrm{~km}$, but it is not continuous along the rainbands. On the other hand, $\mathrm{LH}$ at $7 \mathrm{~km}$ agrees well with the VV. The region with an updraft at $7 \mathrm{~km}$ corresponds to the region with positive $\mathrm{LH}$, implying a phase change from liquid water to ice since the freezing level is around $5 \mathrm{~km}$. Although $\mathrm{LH}$ at $1 \mathrm{~km}$ does not represent the
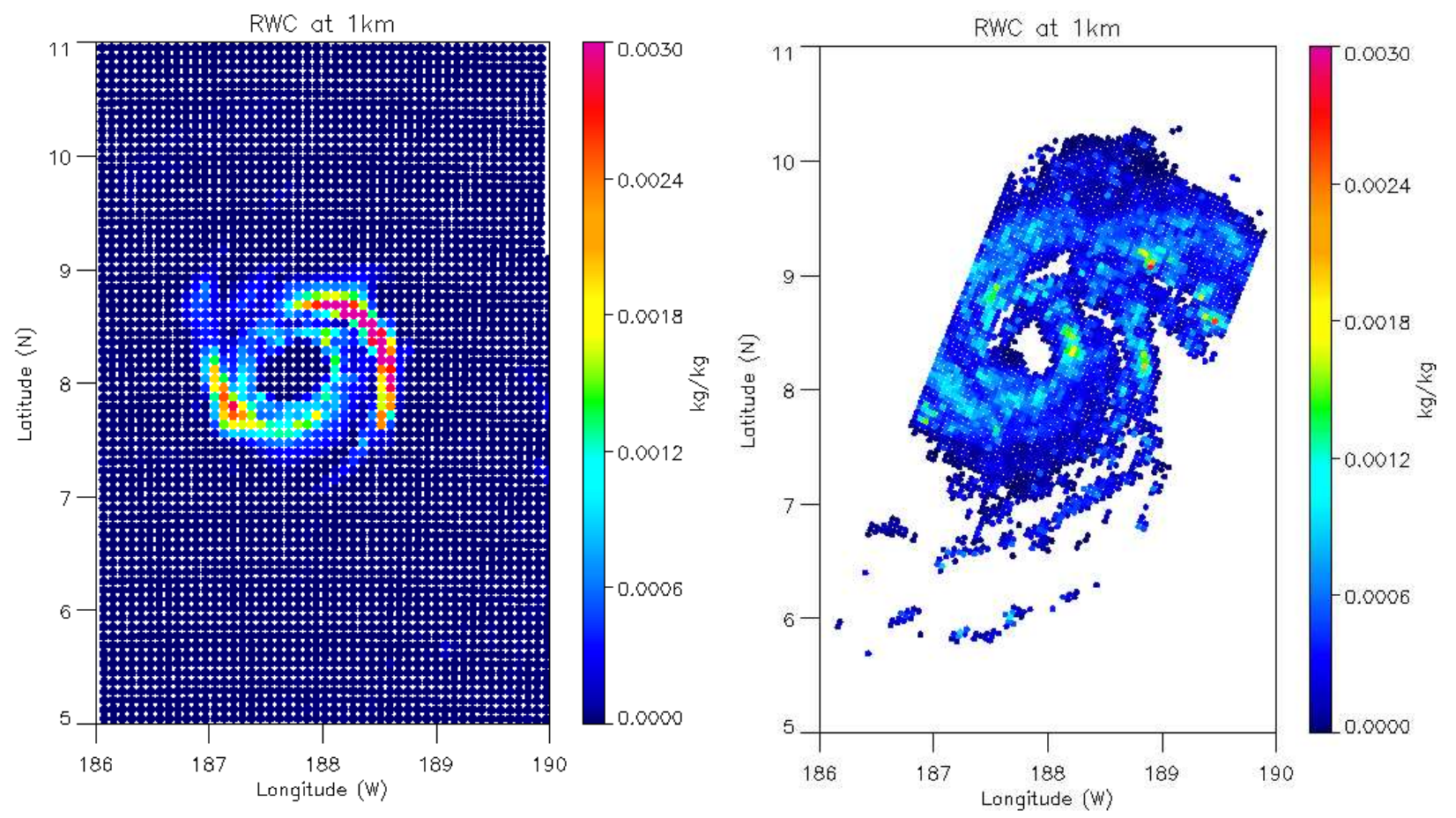

Figure 8 Background (left) and retrieved (right) rain water content horizontal cross-section at $1 \mathrm{~km}$ for Hurricane Pali 
convective rainband features very well, the rain water content agrees well with the observed reflectivity as shown in Figure 8.

Hydrometeors follow the reflectivity pattern most closely as it is directly related to the reflectivity. Precipitating hydrometeors were only examined at the level where they exist (rain at $1 \mathrm{~km}$ and snow and graupel at $7 \mathrm{~km}$ ). At $7 \mathrm{~km}$, snow and graupel (Figure 9 and 10) agree well with the observed scene as well. Overall, the retrieved data looks reasonable to represent the real TC system.
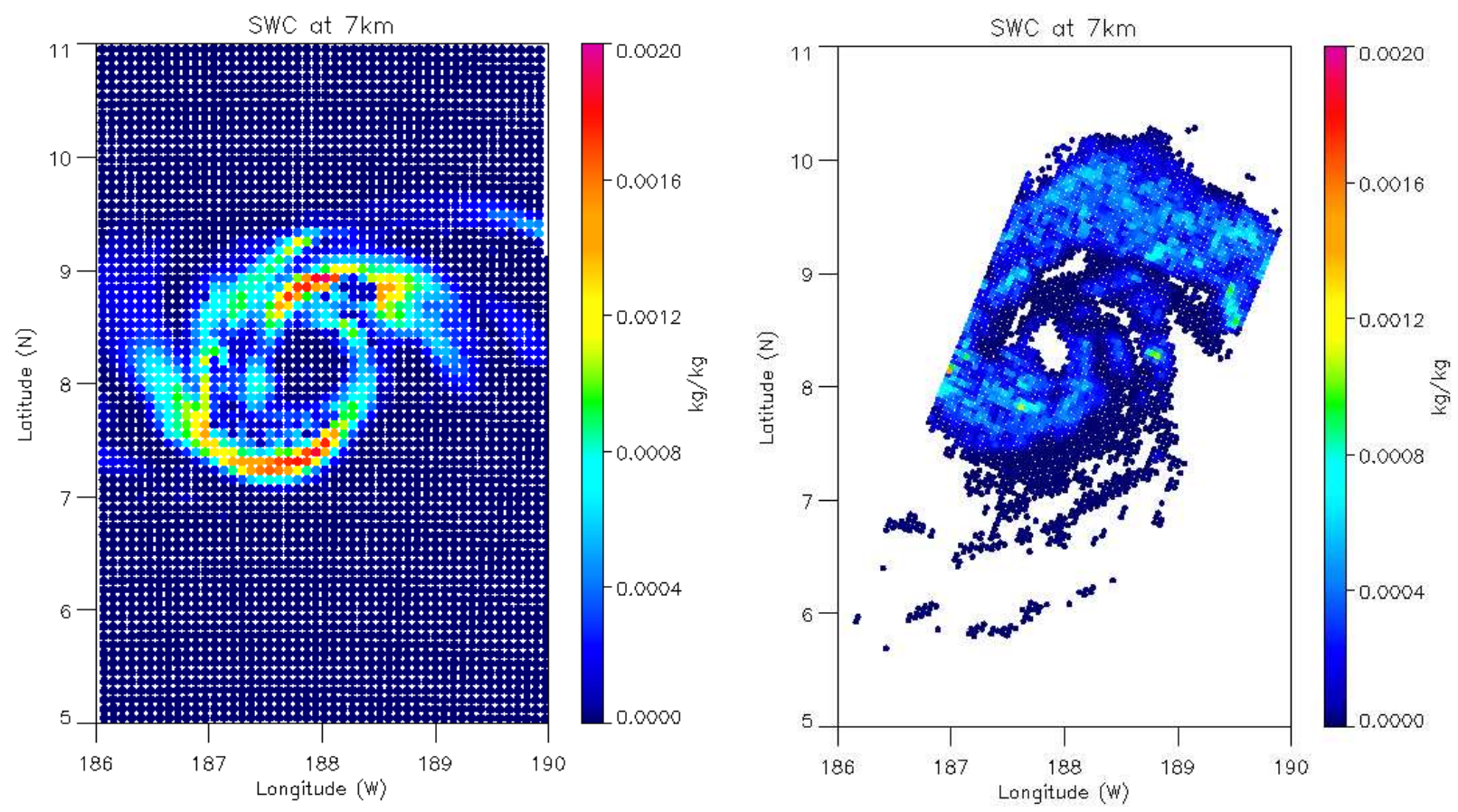

Figure 9 Background (left) and retrieved (right) snow water content horizontal cross-section at $7 \mathrm{~km}$ for Hurricane Pali 

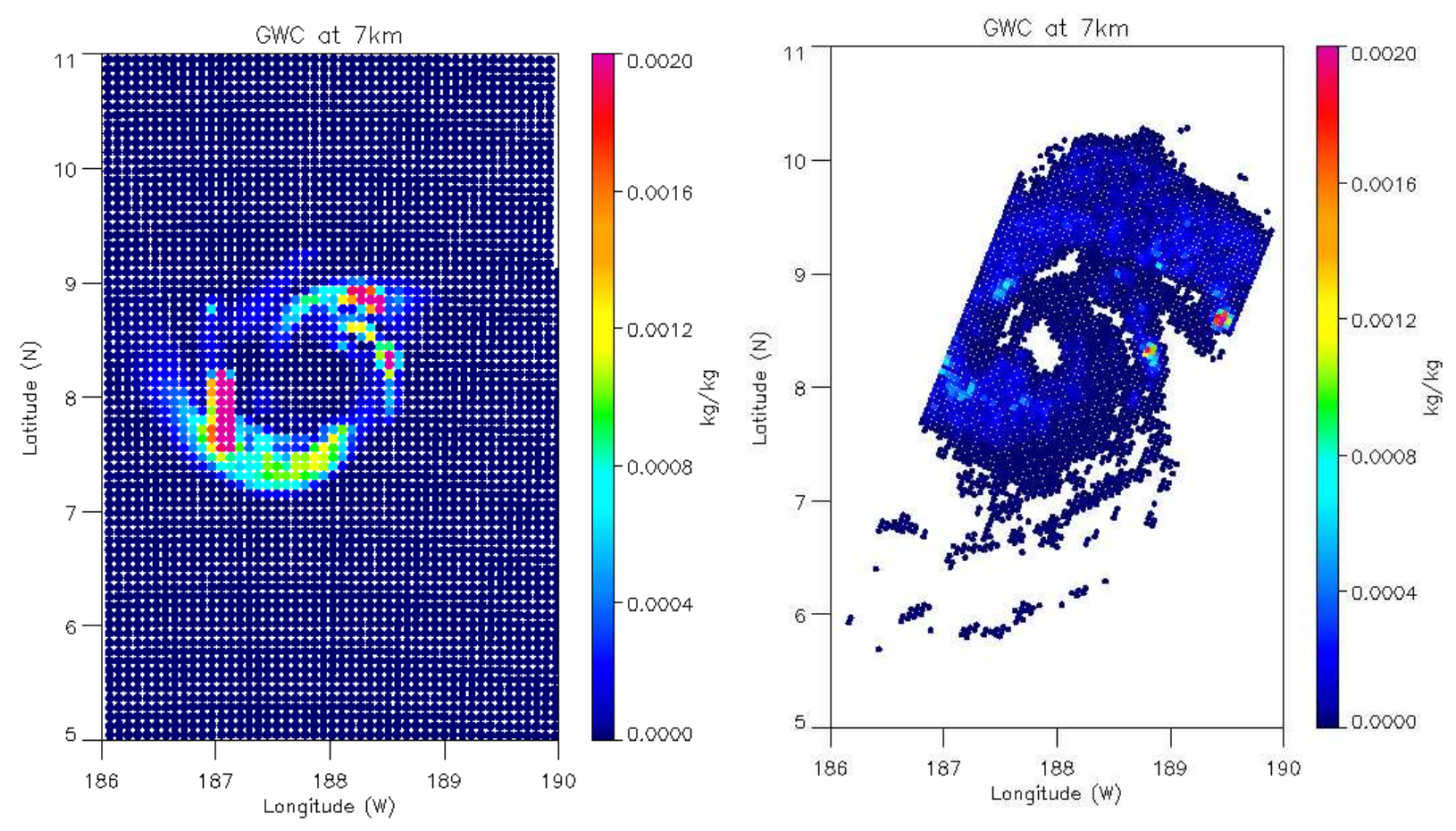

Figure 10 Background (left) and retrieved (right) graupel water content horizontal cross-section at $7 \mathrm{~km}$ for Hurricane Pali

The ensemble forecast was then run at cycle 0 to get the background error covariance matrix and the background state vectors at the analysis time (cycle1). Background data and control variables are identical for the three experiments to make a simple comparison between different assimilated retrievals. At cycle1, three experiments were conducted each assimilating VV, LH, or HYDRO within a 6-hour assimilation window. The number of observations that passed QC for the inner domain with 9km resolution is 85812, 67499, and 134872 for VV, LH, and HYDRO respectively. Individual hydrometeor species have fewer observations that passed QC because they only exist within certain levels, but the total amount of entries is highest for HYDRO when all species are counted. 
The total cost function, that includes observation and background terms, was calculated before and after the DA simulation in order to examine the improvements. A lower cost function means that the difference between observed and modeled variables decreased, thereby improving the quality of the analysis. Cost functions decreased by $27 \%$ (from $3.3341 \times 10^{4}$ to $2.4292 \times 10^{4}$ ), $33 \%$ (from $2.3849 \times 10^{4}$ to $1.5951 \times 10^{4}$ ), and $21 \%$ (from $5.2525 \times 10^{4}$ to $4.1478 \times 10^{4}$ ) for $\mathrm{VV}$, $\mathrm{LH}$, and HYDRO respectively. All of the experiments show substantial improvements after assimilation. Histogram plots of the difference between observation and modeled output before and after DA are shown in Figure 11,12, and 13 for VV, LH, and HYDRO, respectively. Histograms were made with points that had an observation that passed the QC and were assimilated into the system. The edge of the bin is constrained by $3 \sqrt{R}$ which comes from the QC $\left(\frac{|y-H(x)|}{\sqrt{R}}<3\right) . \mathrm{VV}$ shows the most Gaussian-like distribution among the three variables because it has the smallest range of values and the difference between model and observations is naturally centered on zero. Its distribution became more Gaussian-like after DA with a lower frequency of occurrence at the edges and higher frequency around zero. Although the frequency of occurrence of the peak decreased after DA, the sum of frequencies of occurrence for the bins around zero increased markedly, implying that DA successfully adjusted the background values to observed values. Unlike VV, LH and HYDRO have a highly peaked and skewed shapes of the distribution. LH is skewed to the left which might suggest that the scheme is assimilating LH in the region where ice hydrometeors are overestimated. HYDRO, on the other hand, is somewhat skewed to the right which implies that grids where there is more water content in the observation are assimilated more than grids with larger water content in the background. LH and HYDRO began with an error distribution that is non-Gaussian, thus contradicting the assumption in MLEF. The residuals are nonetheless improved after DA. 

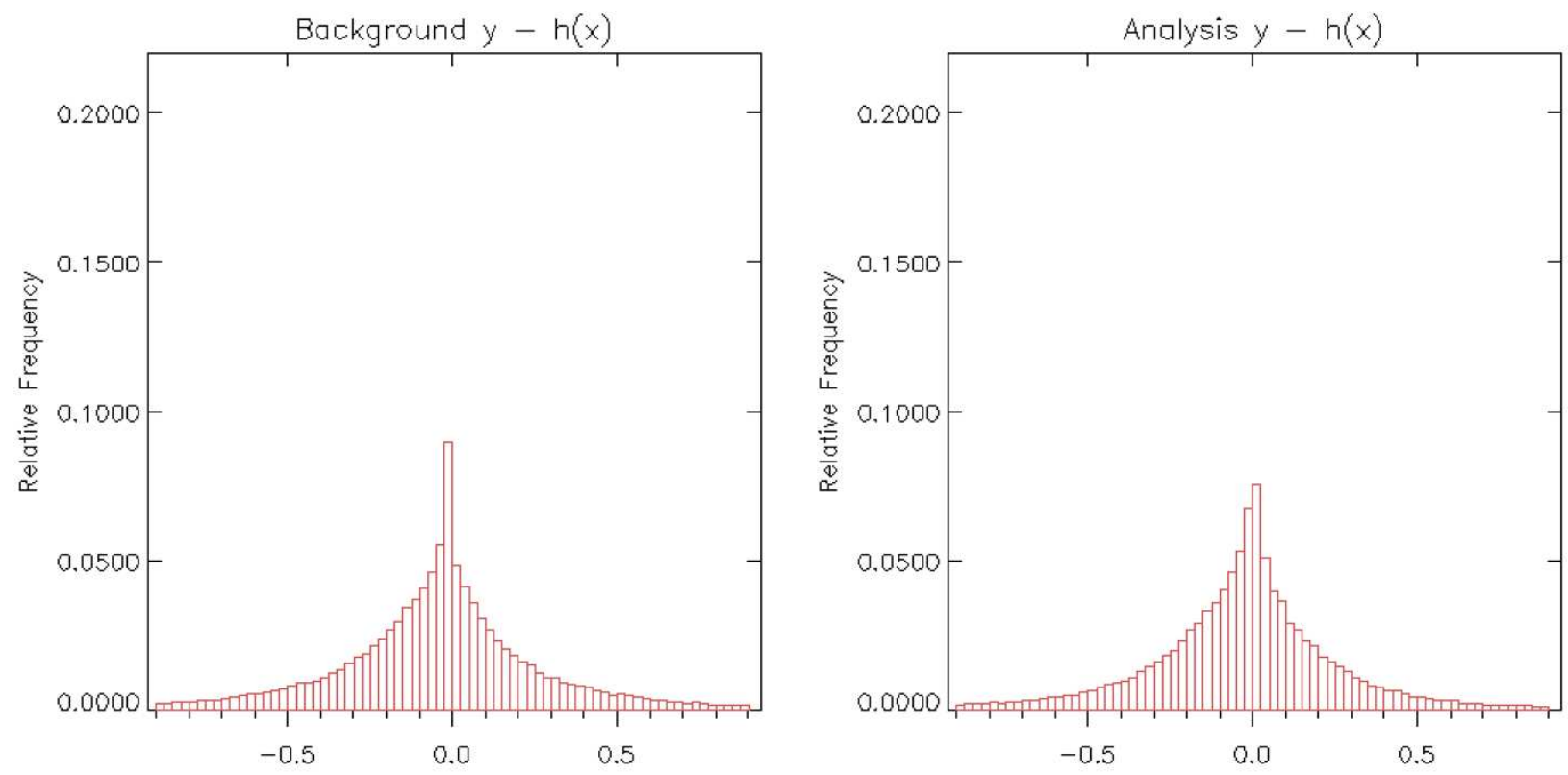

Figure 11 Histograms of observation-background for VV before (left) and after (right) DA simulation
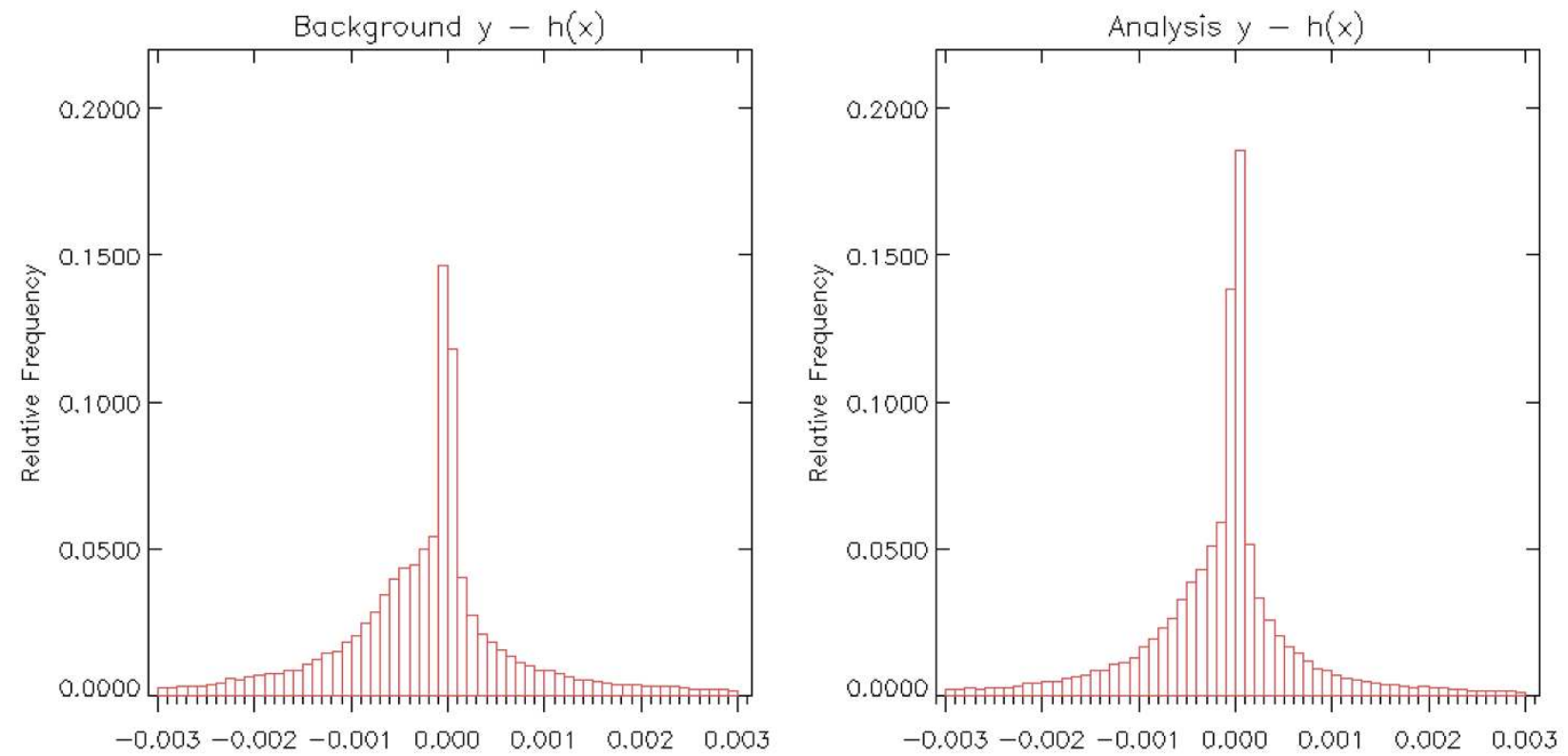

Figure 12 Histograms of observation-background for LH before (left) and after (right) DA simulation 

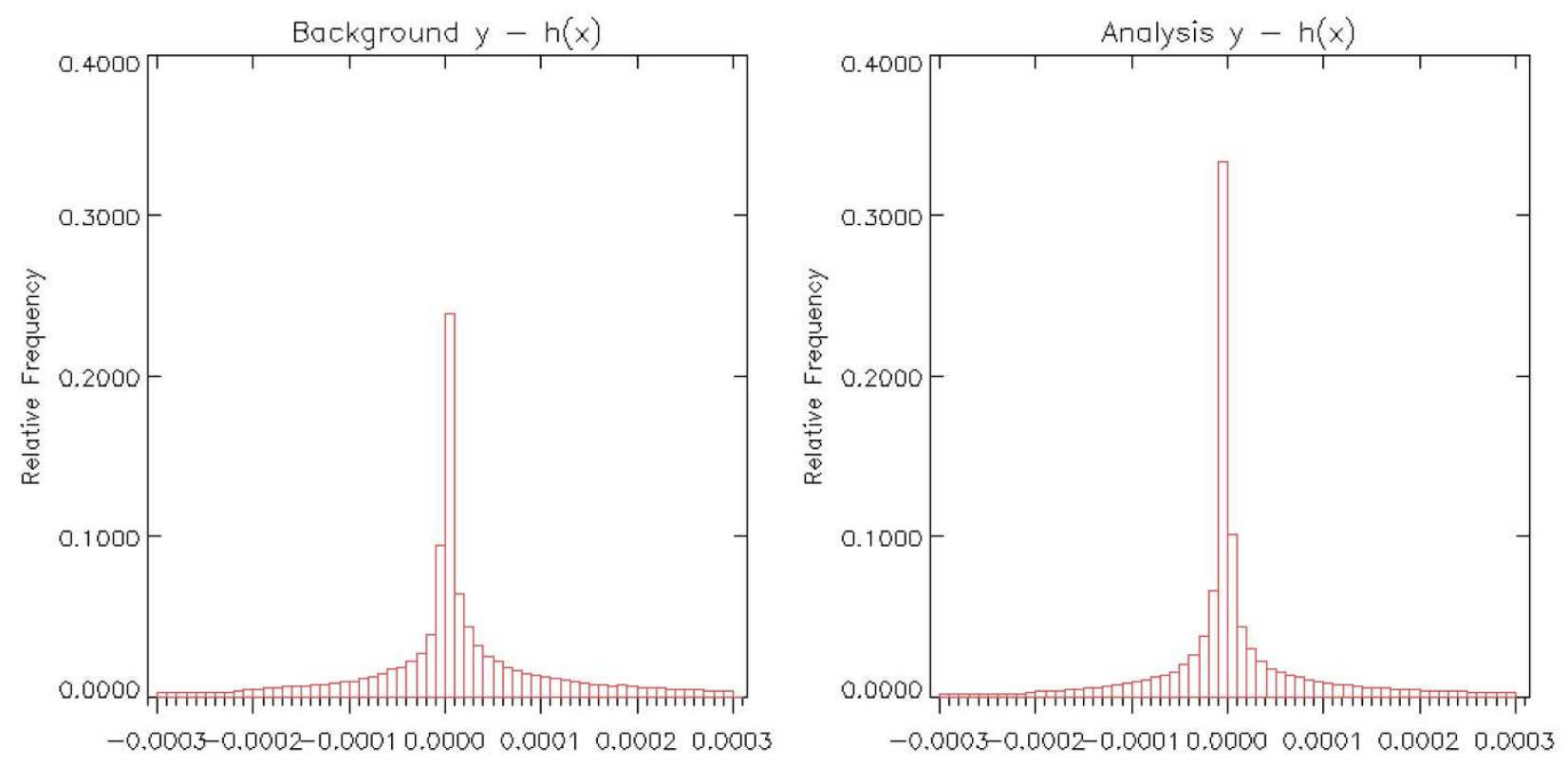

Figure 13 Histograms of observation-background for HYDRO before (left) and after (right) DA simulation

Analysis vectors produced after the DA experiments were examined with independent observations for an objective validation. Brightness temperature from GMI can be used for independent verification purposes. For a comparison between the observed $T_{b}$ and the $T_{b}$ calculated based on the analysis and background, $\mathrm{T}_{\mathrm{b}}$ at $89 \mathrm{GHz}$ (vertical polarization) was used because this channel is sensitive to hydrometeors, especially in the ice phase. In Figure 14, the model background correctly created the convective core of the hurricane in the observed location, but it overestimated ice hydrometeors around the rainbands, and did not create the rainband around at $9^{\circ} \mathrm{N}$. Overestimation of ice hydrometeors is evidenced by lower $\mathrm{T}_{\mathrm{b}}$ caused by too much scattering of radiation away from the viewing direction. Figure 15 is the same brightness temperature map but with VV, LH, and HYDRO run. VV seemed to create the upper 
part of the rainband but still significantly overestimates the amount of ice scattering as in the background. In addition, it moved the lower part of the rainband southward which should have been moved northward. By contrast, the LH run reduced the amount of ice scattering considerably and created the upper rainband. It even tried to reshape the lower part of the rainband. HYDRO showed a similar pattern as LH but with less scattering at the lower rainband and higher scattering at the upper rainband.
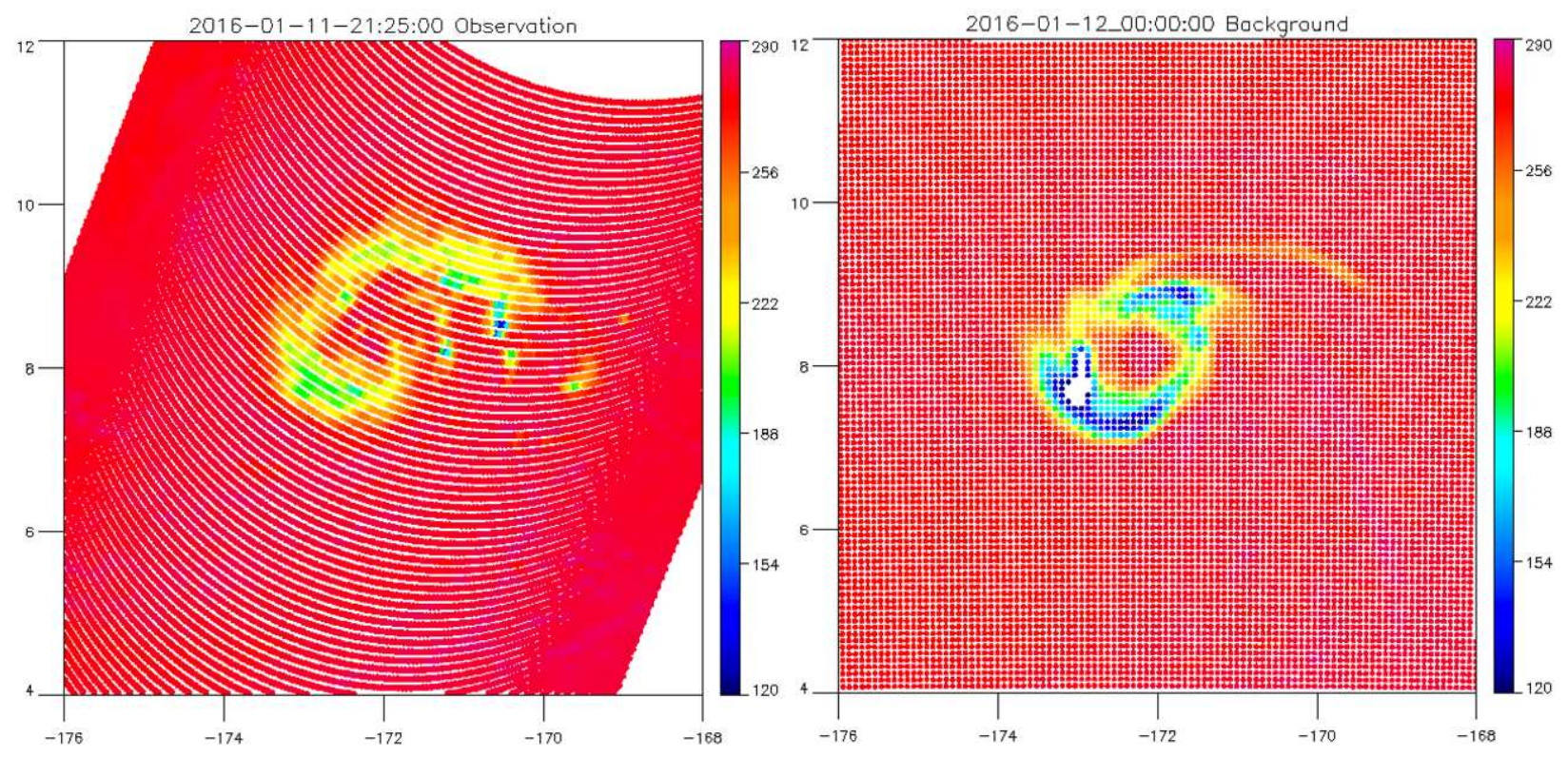

Figure 14 Brightness temperature map of observation and the background at $89 \mathrm{GHz} \mathrm{V}$ for Hurricane Pali 

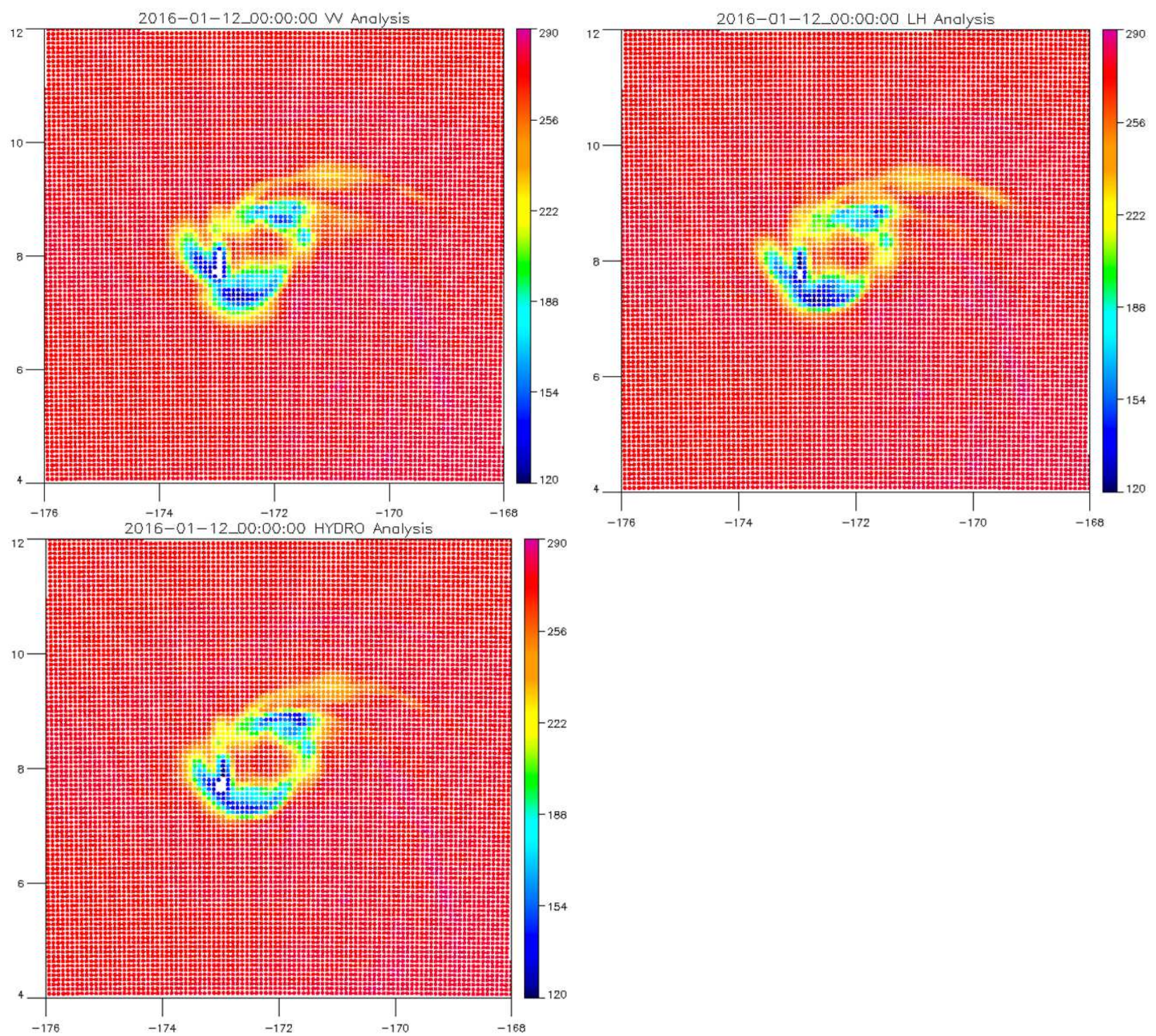

Figure 15 Brightness temperature map of VV, LH, and HYDRO at $89 \mathrm{GHz}$ V for Hurricane Pali

In addition to the $\mathrm{T}_{\mathrm{b}}$, DPR reflectivity can also be used for validation data although it was used in the retrieval of the assimilated parameters. It is nonetheless useful in the sense that it can be evaluated at different levels. Figure 16 and 17 show reflectivity before and after the DA experiments at $1 \mathrm{~km}$ and $7 \mathrm{~km}$ respectively. Background reflectivity at $1 \mathrm{~km}$ appears discontinuous at the edge of the rainbands. LH and HYDRO improved the simulation by creating trailing rainbands. VV also formed a rainband but it removed the northwest portion of it. At $7 \mathrm{~km}$, all three experiments showed similar patterns between the three experiments. They produced ice 
hydrometeors in the north east part of the rainband but, at the same time, they created unnecessary ice at the south of the rainband around $7^{\circ} \mathrm{N}$. One thing to note here is that the results for LH and HYDRO look very similar both at $1 \mathrm{~km}$ and $7 \mathrm{~km}$.
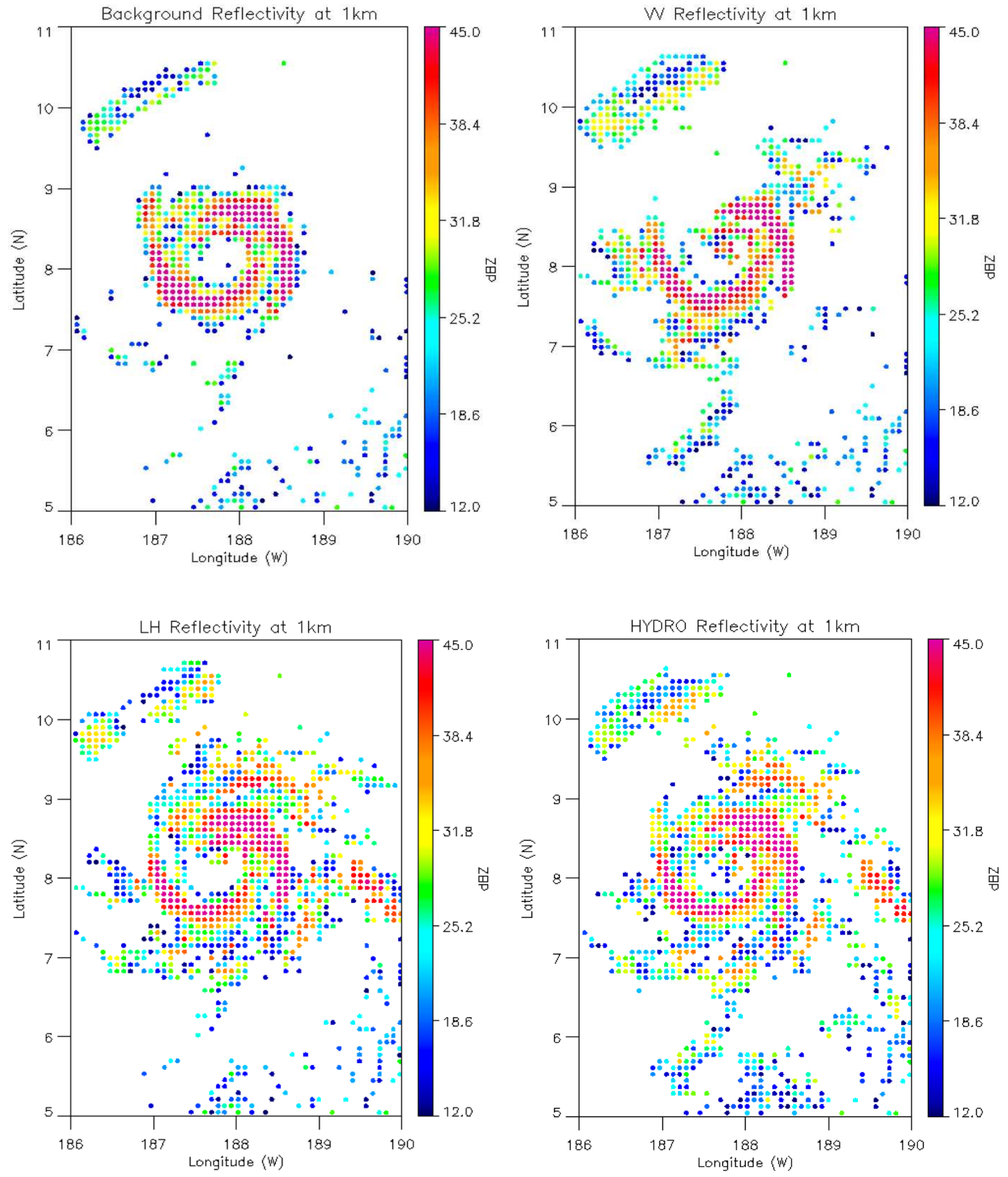

Figure 16 Horizontal cross-section of reflectivity at $1 \mathrm{~km}$ for Background. VV. LH, and HYDRO 

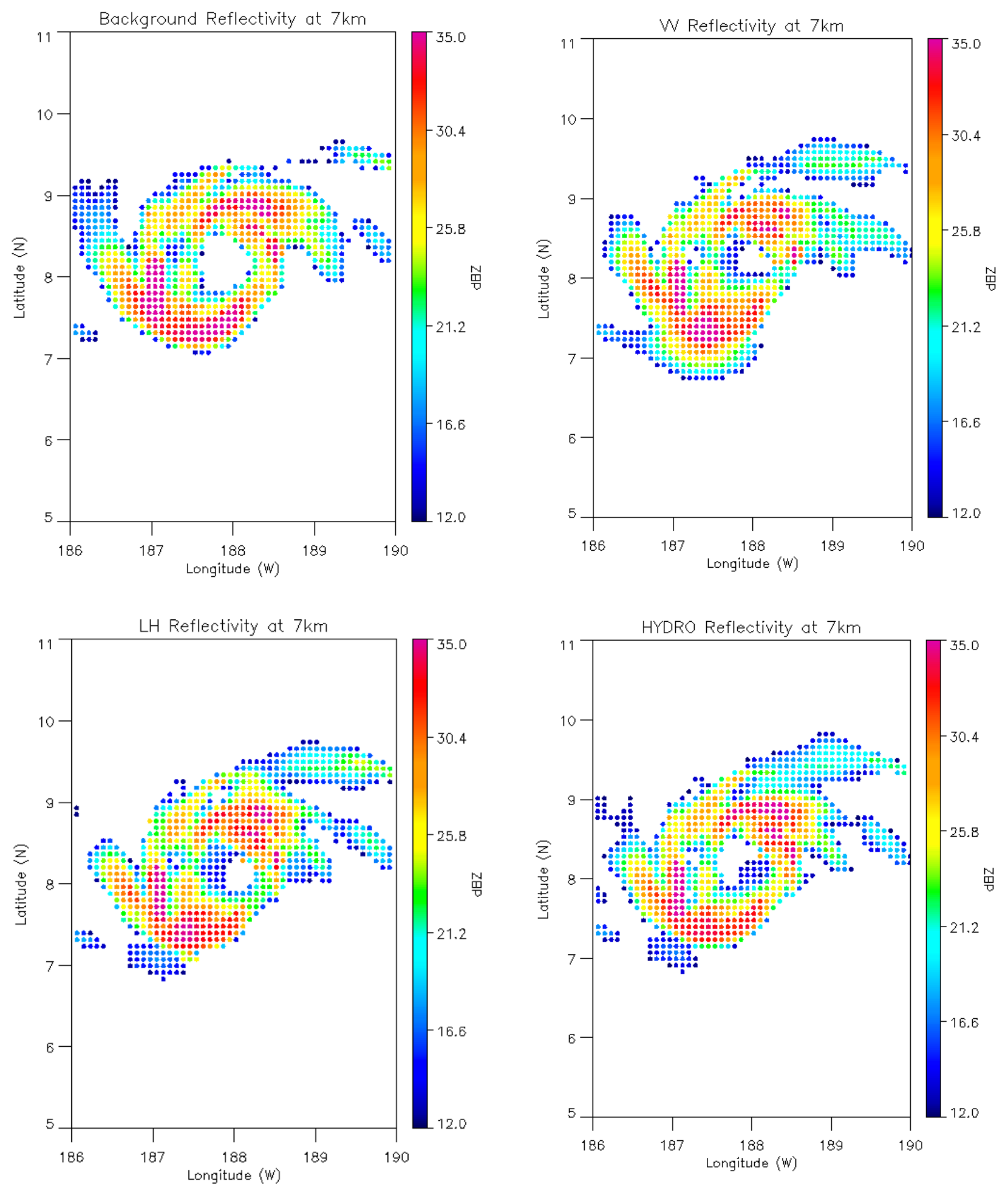

Figure 17 Horizontal cross-section of reflectivity at $7 \mathrm{~km}$ for Background, VV, LH, and HYDRO 
Figures 18, 19, and 20 provide a quantitative assessment of the improved analysis for the VV, LH, and HYDRO assimilation experiments respectively. The horizontal axis in each plot displays the absolute difference between the observations and the background reflectivity while the vertical axis is the difference between the observations and the analysis. Points to the right of the one-to-one line therefore show an improved analysis field. Two figures are shown for each experiment: one that includes all of the points and one that excludes points on the one-to-one line. Points on the one-to-one line might appear to be unaffected by the DA procedure, but it is more likely that the change is very subtle and not detectable in the figure. An alternative explanation for the large number of points along the one-to-one line could be that observation errors were too large compared to forecast errors, and that the model thus tends to ignore these observations. All three experiments showed some improvements. In the VV experiment, 13.0\% of the points improved in the analysis while $6.3 \%$ became worse and the remaining $80.7 \%$ was unchanged. Of those that changed, $67.5 \%$ showed improved and $32.5 \%$ retrograded. In the LH experiment, $12.9 \%$ of the total analysis improved. Excluding the points on the one-to-one line, the right plot in Figure 19 shows 70\% improvement. HYDRO experiment also shows $70 \%$ of improvement without the unchanged points. The number of points that had passed the QC was larger in the HYDRO run than with LH, making HYDRO the most influential variable. The reason why HYDRO had the most positive impact on reducing the discrepancy in reflectivity might be because hydrometeor water contents are directly related to the reflectivity calculation as an input in the RTM. Positive effect on LH seems comparable to HYDRO even though LH is not an input to the RTM. 

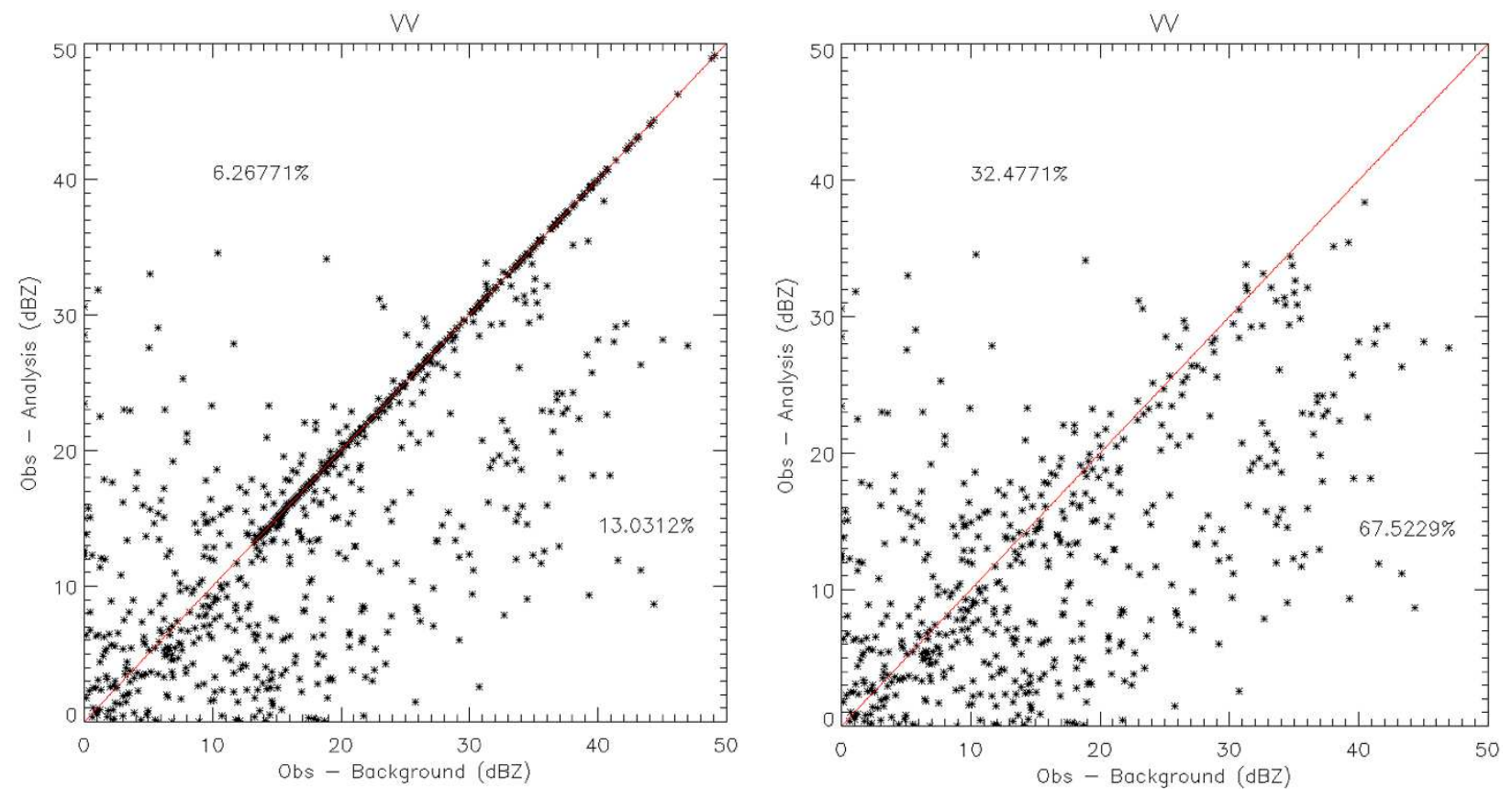

Figure 18 Scatter plot of difference between observation and analysis against difference between observation and background for VV including (left) and excluding(right) points that did not change
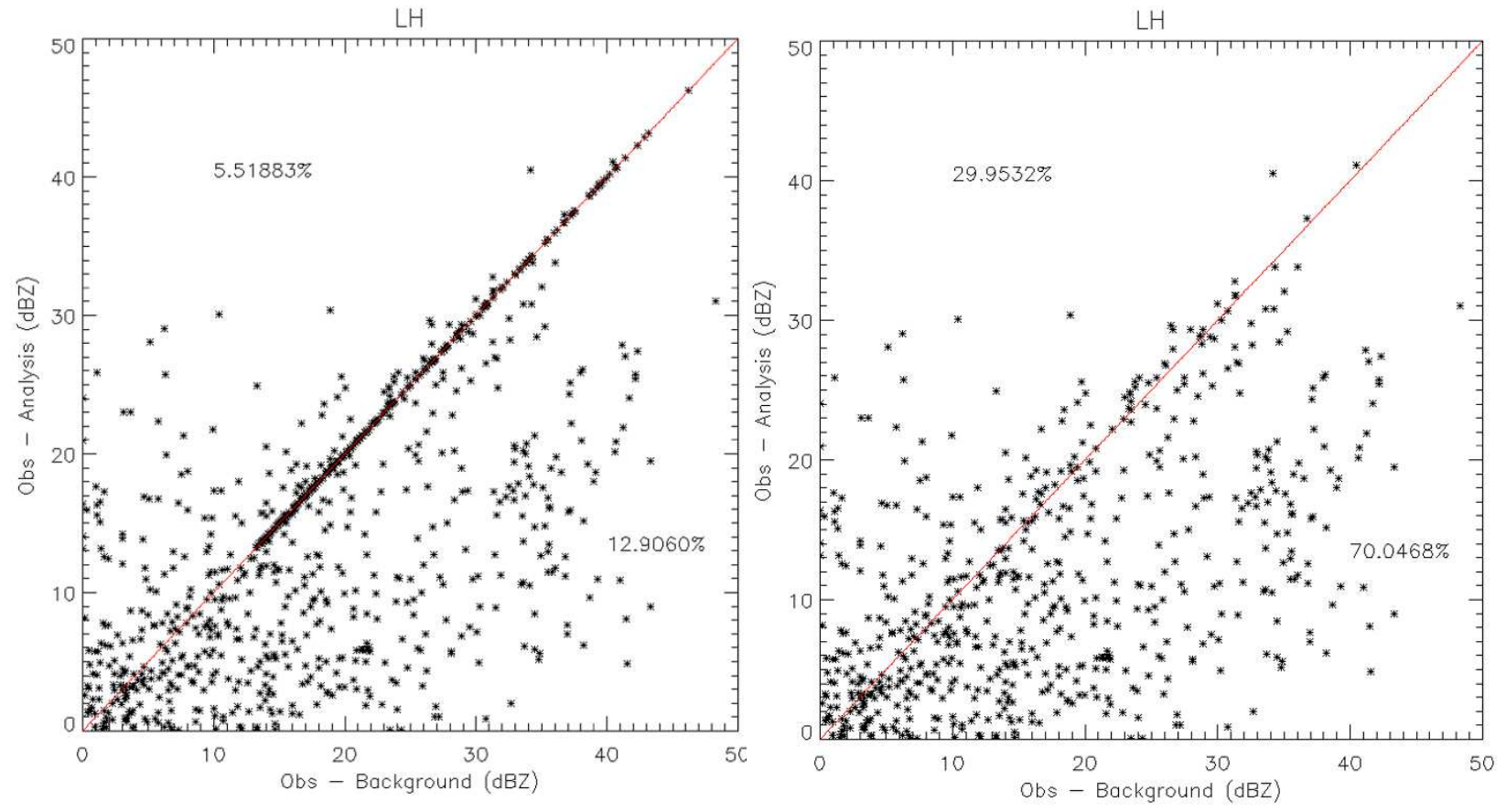

Figure 19 Scatter plot of difference between observation and analysis against difference between observation and background for LH including (left) and excluding (right) points that did not change 

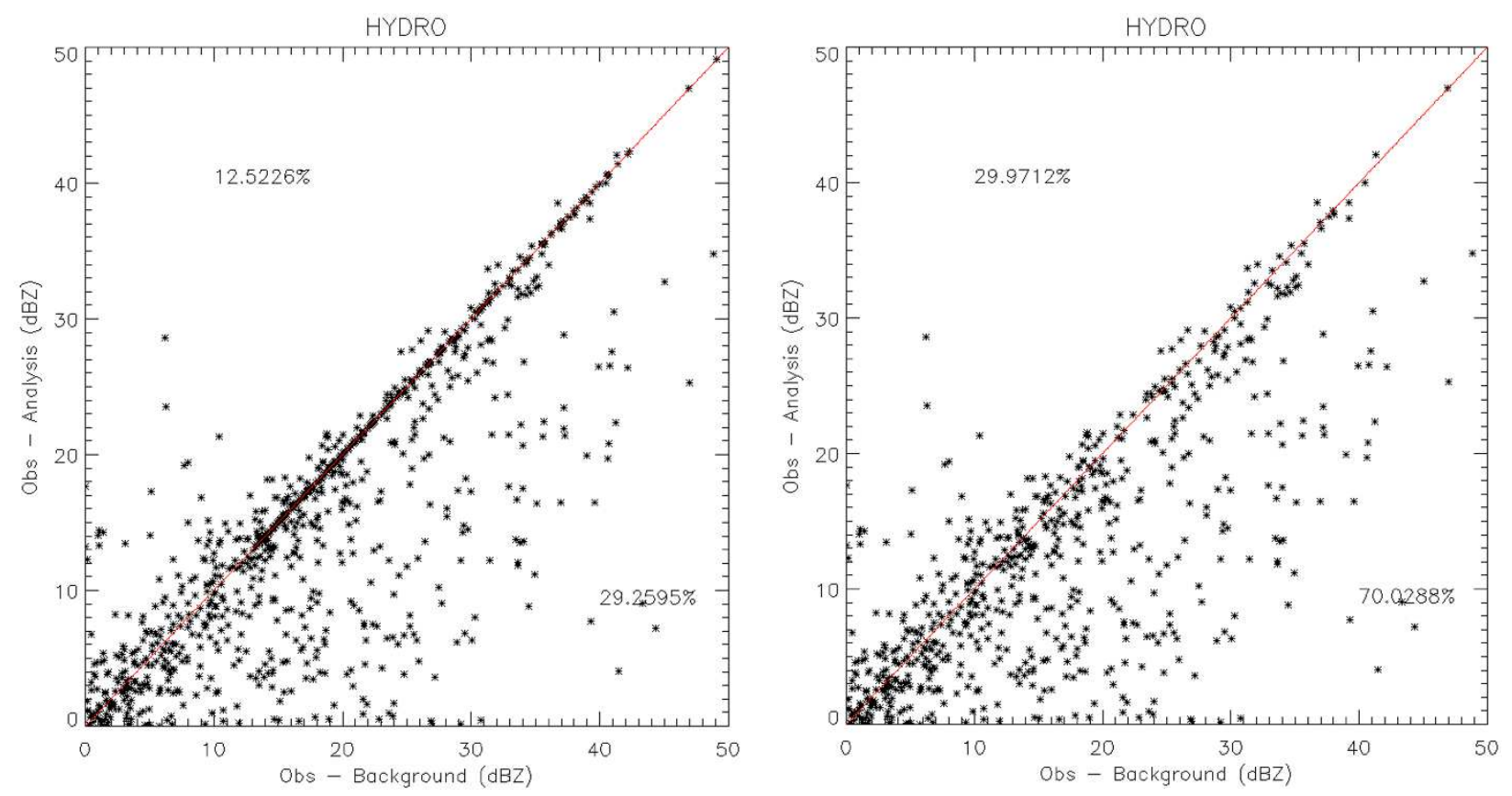

Figure 20 Scatter plot of difference between observation and analysis against difference between observation and background for HYDRO including (left) and excluding (right) points that did not change

\subsection{Results in Hurricane Jimena case}

The same experiments were conducted for Hurricane Jimena starting with the creation of a retrieved data set. Jimena was a bigger and stronger hurricane than Pali as is shown in Figure 21. This can be supported by a map of VV in Figure 22 showing positive values in all the identifiable rainbands in the retrieved profiles. The retrieved field captured the hurricane reasonably well in most of the field. Yet, convective features in LH (release of the LH), corresponding to high reflectivity, are not represented well at $1 \mathrm{~km}$ in Figure 23 even though rain water contents are high along the rainbands in Figure 24. Most of the regions in TC Jimena, except for a few grids on the core, can be considered to have stratiform features based on a significant amount of snow (Figure 25) rather than graupel (Figure 26) and a transition of a sign for LH from bottom to top. The background model field, on the other hand, had a larger 
convective core and narrower rainbands. Therefore, a key point in Jimena's case would be whether DA experiments were able to reduce the core size and create a broader rainband structure.
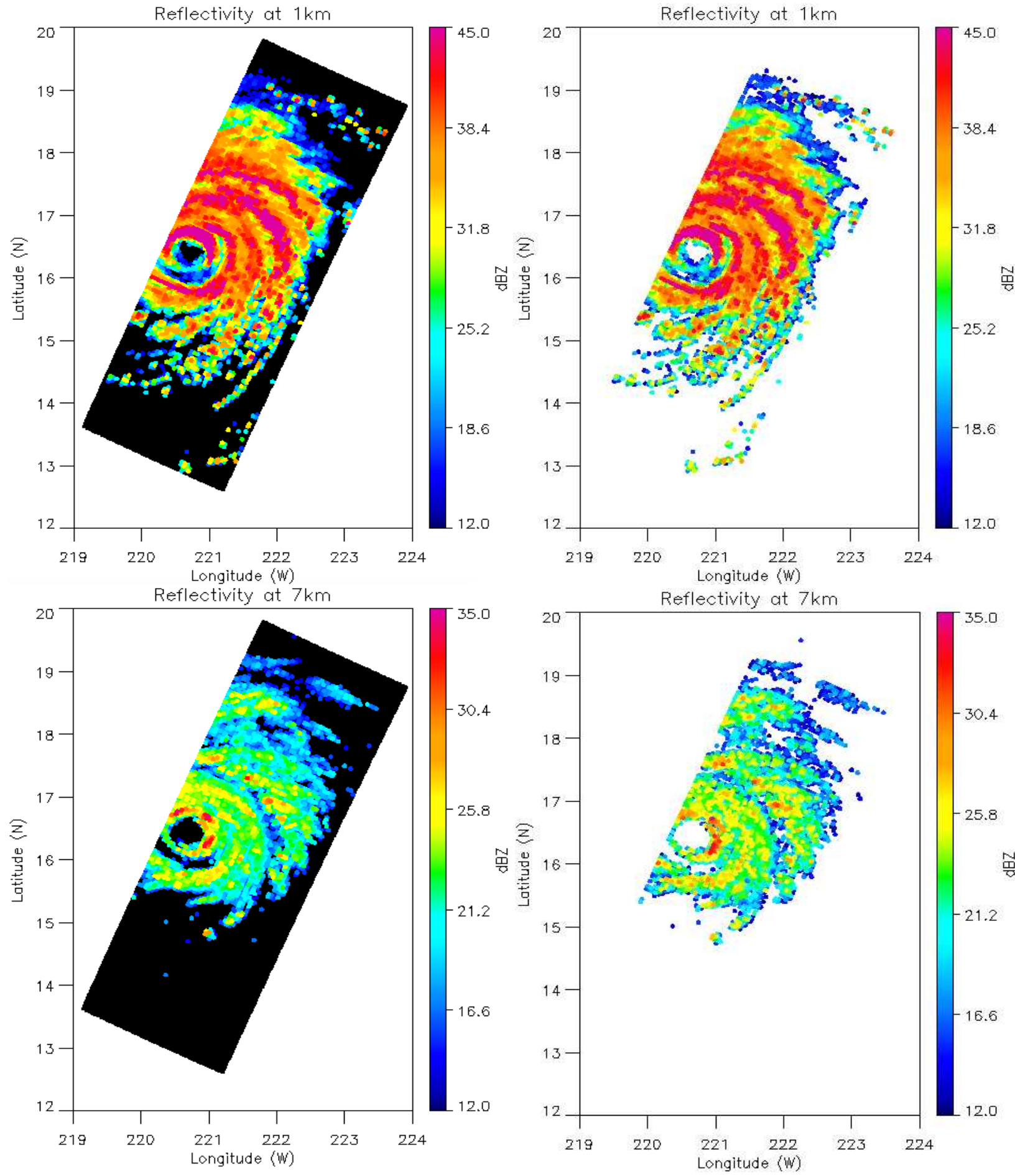

Figure 21 Observed (left) and retrieved (right) reflectivity horizontal cross-section at $1 \mathrm{~km}$ (upper) and $7 \mathrm{~km}$ (lower) for Hurricane Jimena 

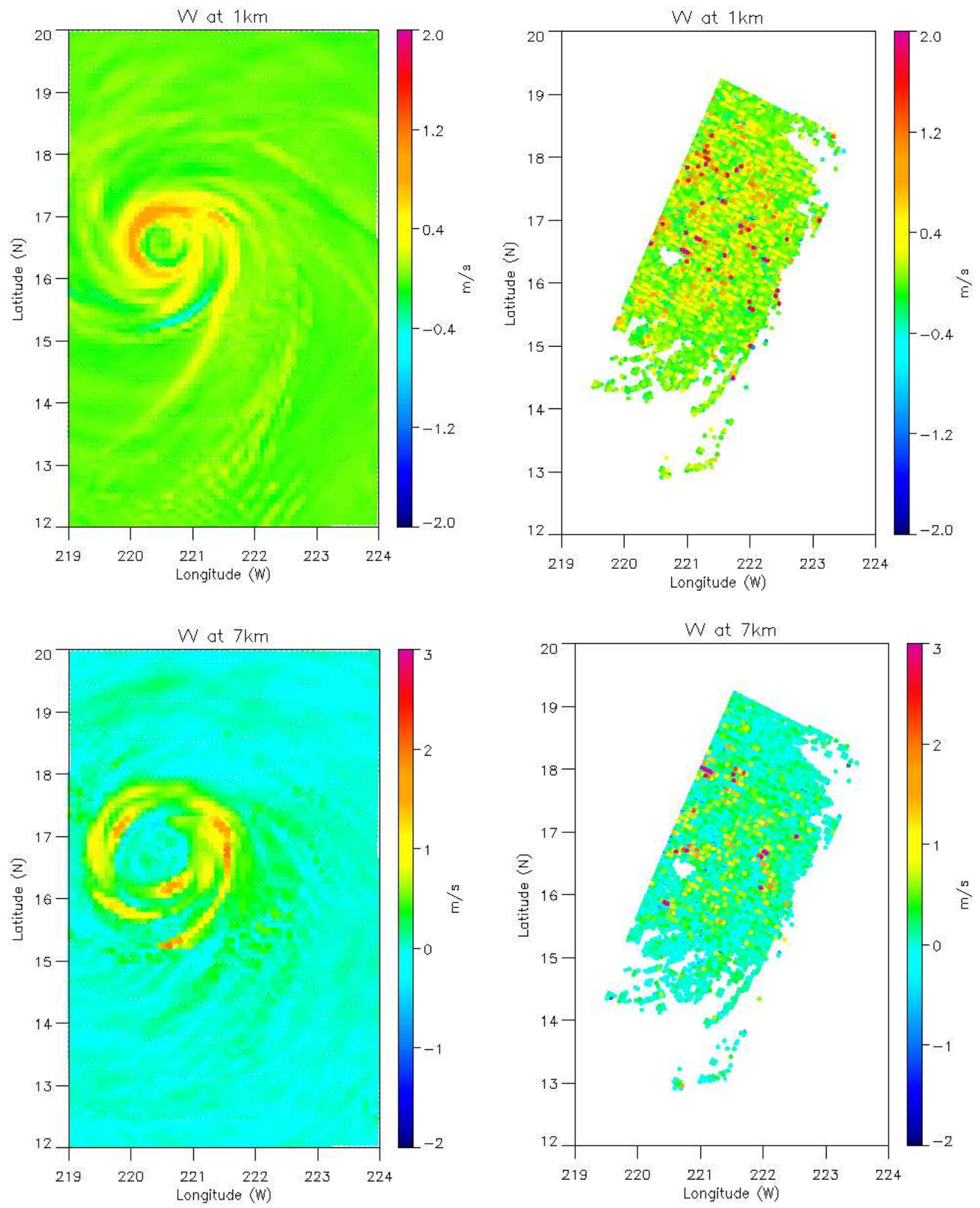

Figure 22 Background(left) and retrieved (right) vertical velocity horizontal cross- section at $1 \mathrm{~km}$ (upper) and $7 \mathrm{~km}$ (lower) for Hurricane Jimena 

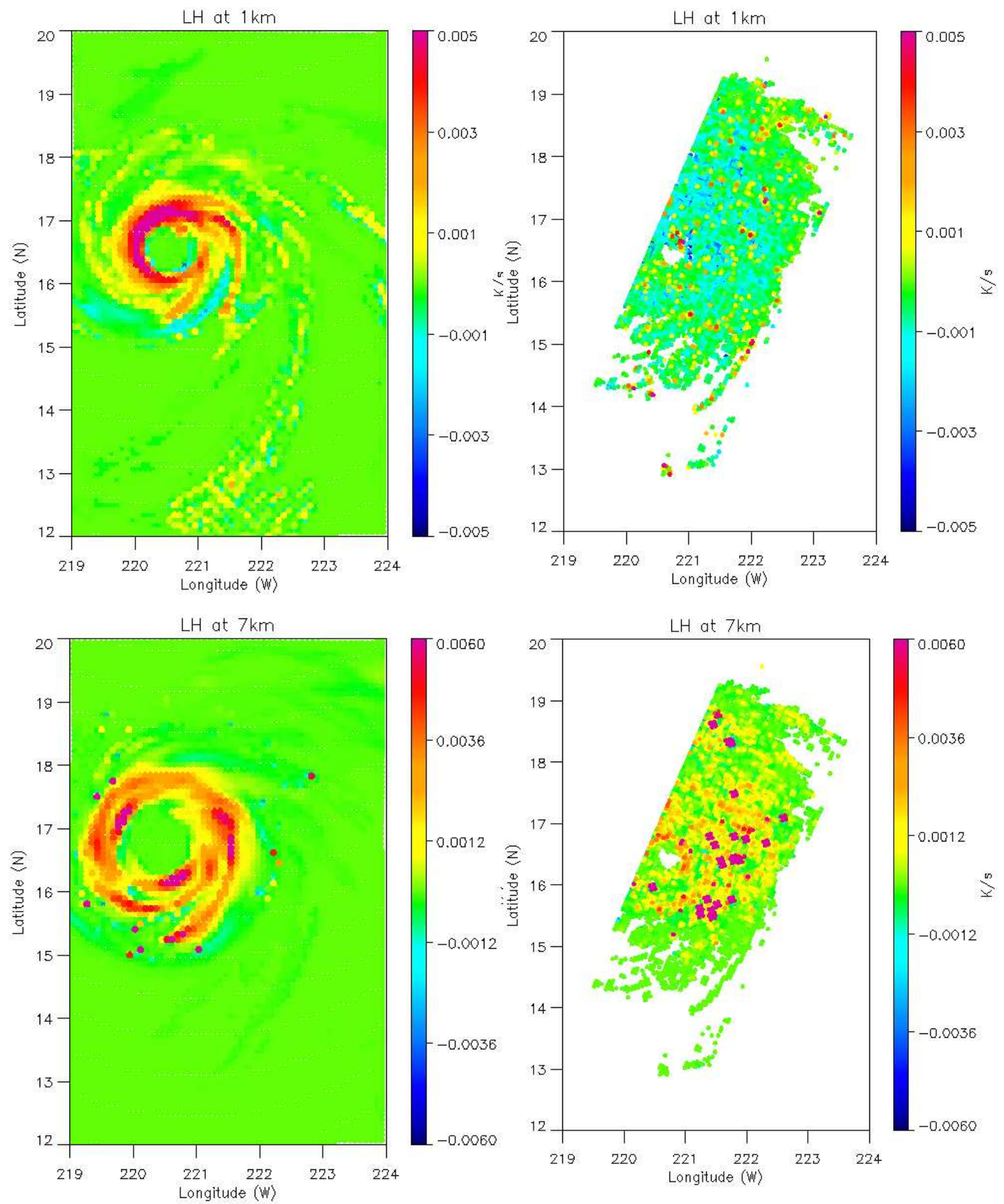

Figure 23 Background(left) and retrieved (right) latent heating horizontal cross- section at $1 \mathrm{~km}$ (upper) and $7 \mathrm{~km}$ (lower) for Hurricane Jimena 

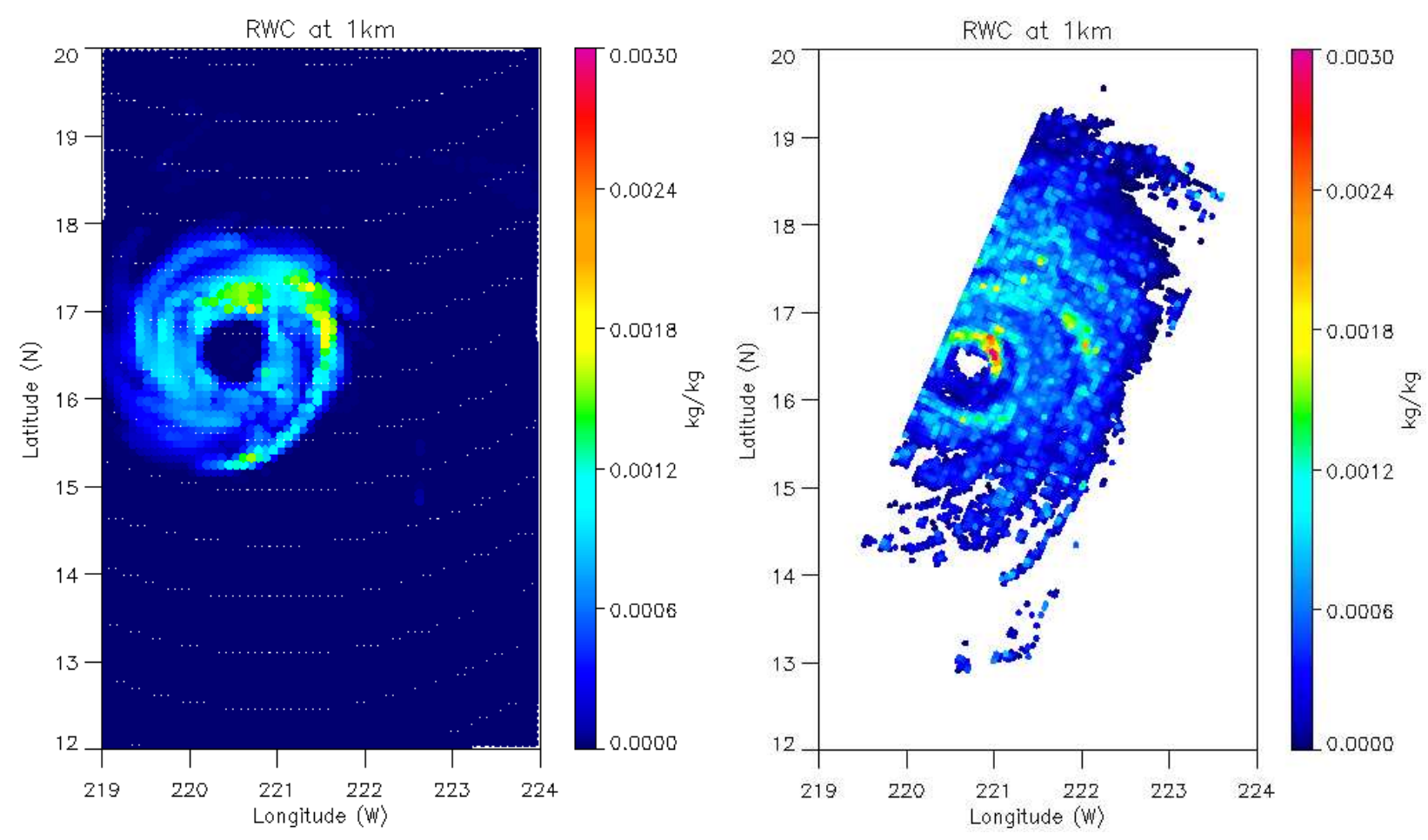

Figure 24 Background(left) and retrieved (right) rain water content horizontal cross-section at $1 \mathrm{~km}$
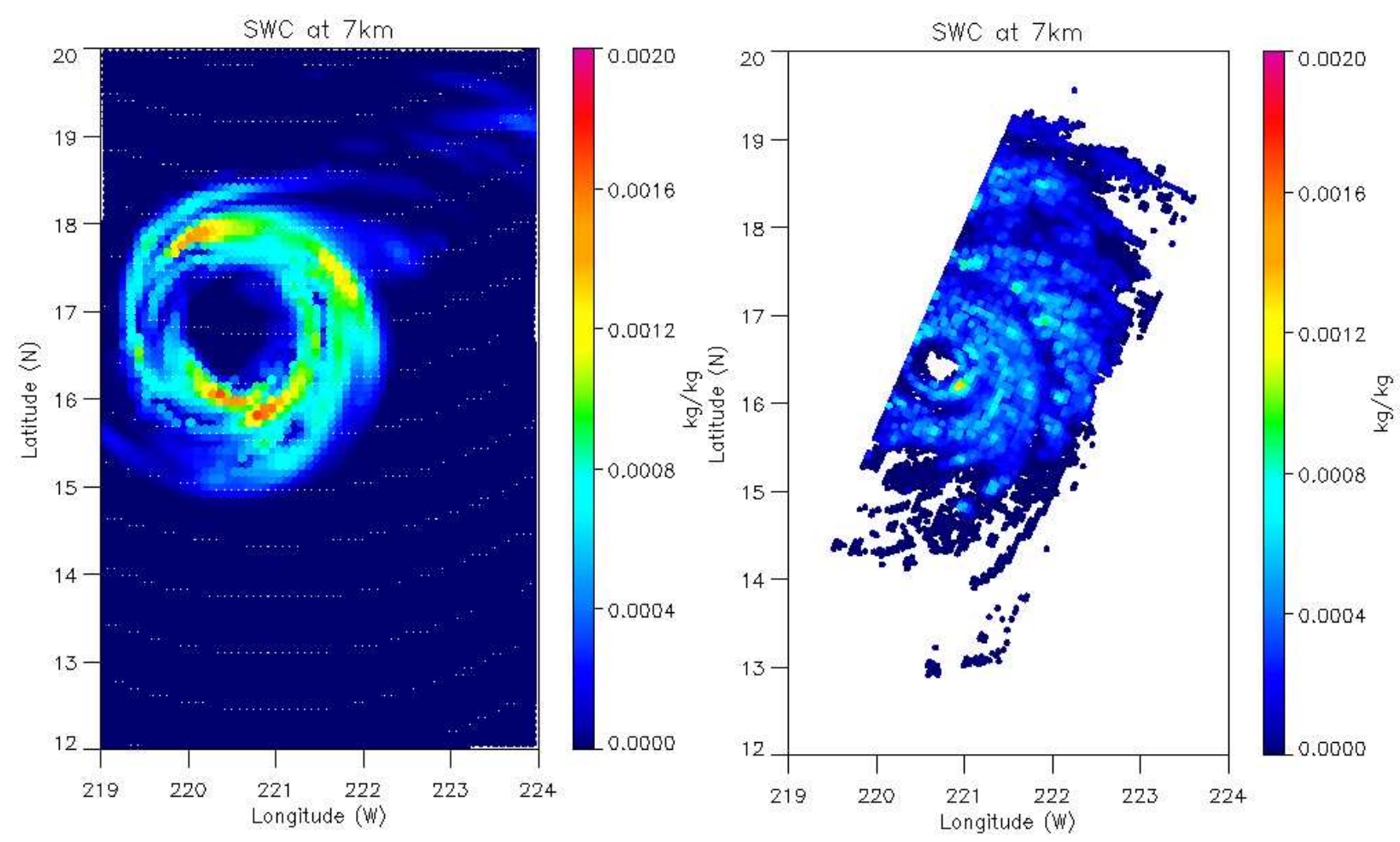

Figure 25 Background(left) and retrieved (right) snow water content horizontal cross-section at $7 \mathrm{~km}$ 

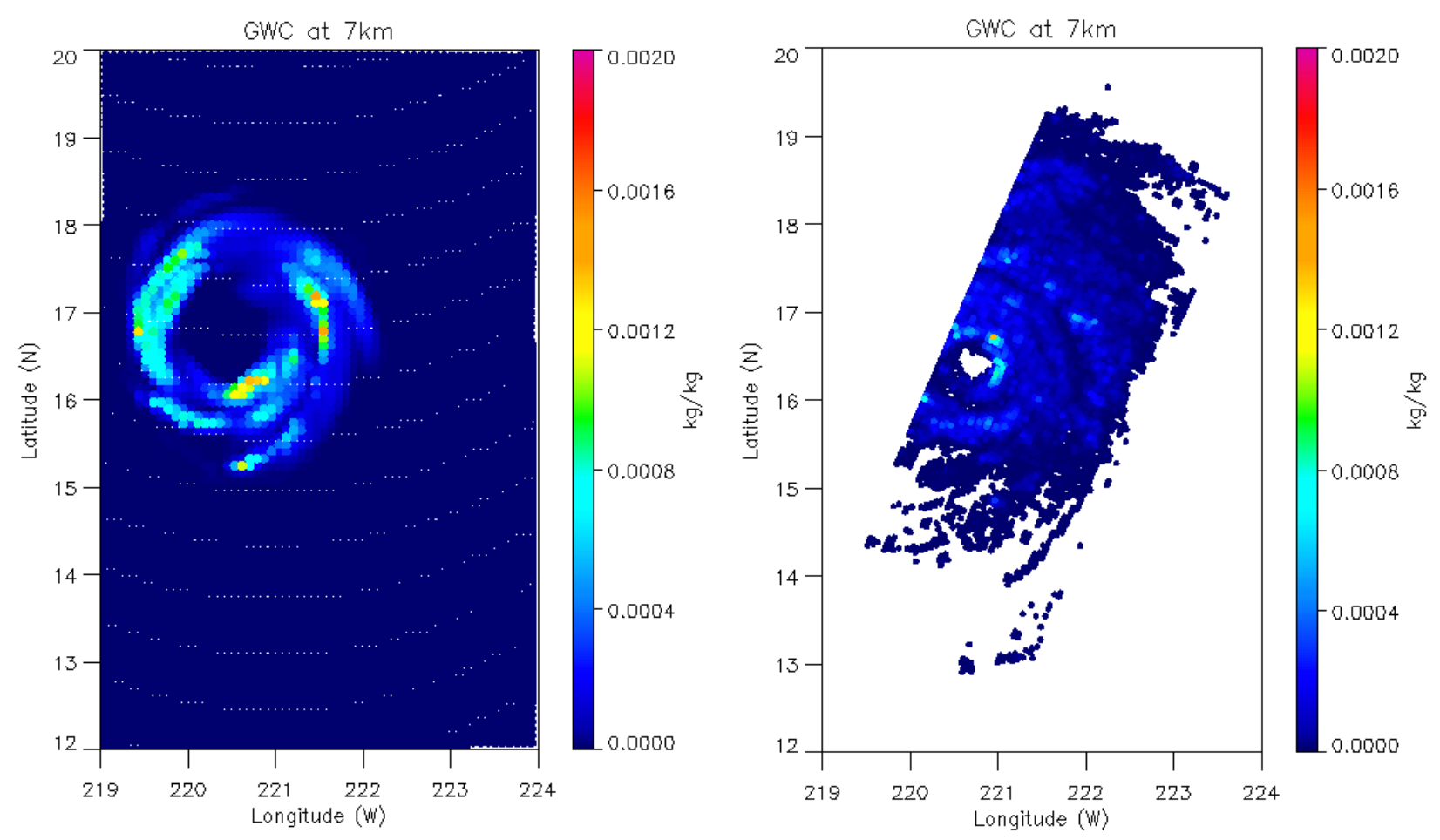

Figure 26 Background(left) and retrieved (right) graupel water content horizontal cross-section at $7 \mathrm{~km}$

Histograms of observed minus simulated parameters in Figure 27, 28, and 29 show similar patterns as with the Pali case: mostly Gaussian-like distribution in VV, skewed to the left in $\mathrm{LH}$, and skewed to the right in HYDRO. By looking at the brightness temperature at $89 \mathrm{GHz}$ in Figure 30, we can see that all of the experiments tried to reduce the convective core size and the ice scattering in the rainband. For evaluating the vertical structure of the TC system after the DA experiments, horizontal cross sections of reflectivity are shown in Figure 31 (at 1km) and 32 (at $7 \mathrm{~km})$. The background simulation at $1 \mathrm{~km}$ is not well developed in the north and east part of the system. At $7 \mathrm{~km}$, it has the higher reflectivity than the observations in the overall rainbands and it is completely missing the north part of the rainbands $\left(18.5^{\circ} \mathrm{N}\right)$. All three experiments had a positive impact at higher levels by lowering ice contents, and thus the reflectivity, but creating 
ice hydrometeors on the North part of the system. However, VV did not have much impact at the lower levels while the other two experiments expanded the rainbands. The LH and HYDRO assimilation experiments showed similar patterns regarding reflectivity just like in the Pali experiments. The reflectivity data was again plotted on a scatter plot to evaluate the improvements quantitatively. The percentage of data that contributed to lower difference between observed and analysis excluding points on the one-to-one line was $67.8 \%, 78.5 \%$, and $78.2 \%$ of the pixels that showed change for VV, LH, and HYDRO (Figure 33, 34, and 35) respectively. Numerical results show that the LH had the most improvement but percentages in LH and HYDRO are comparable to each other.
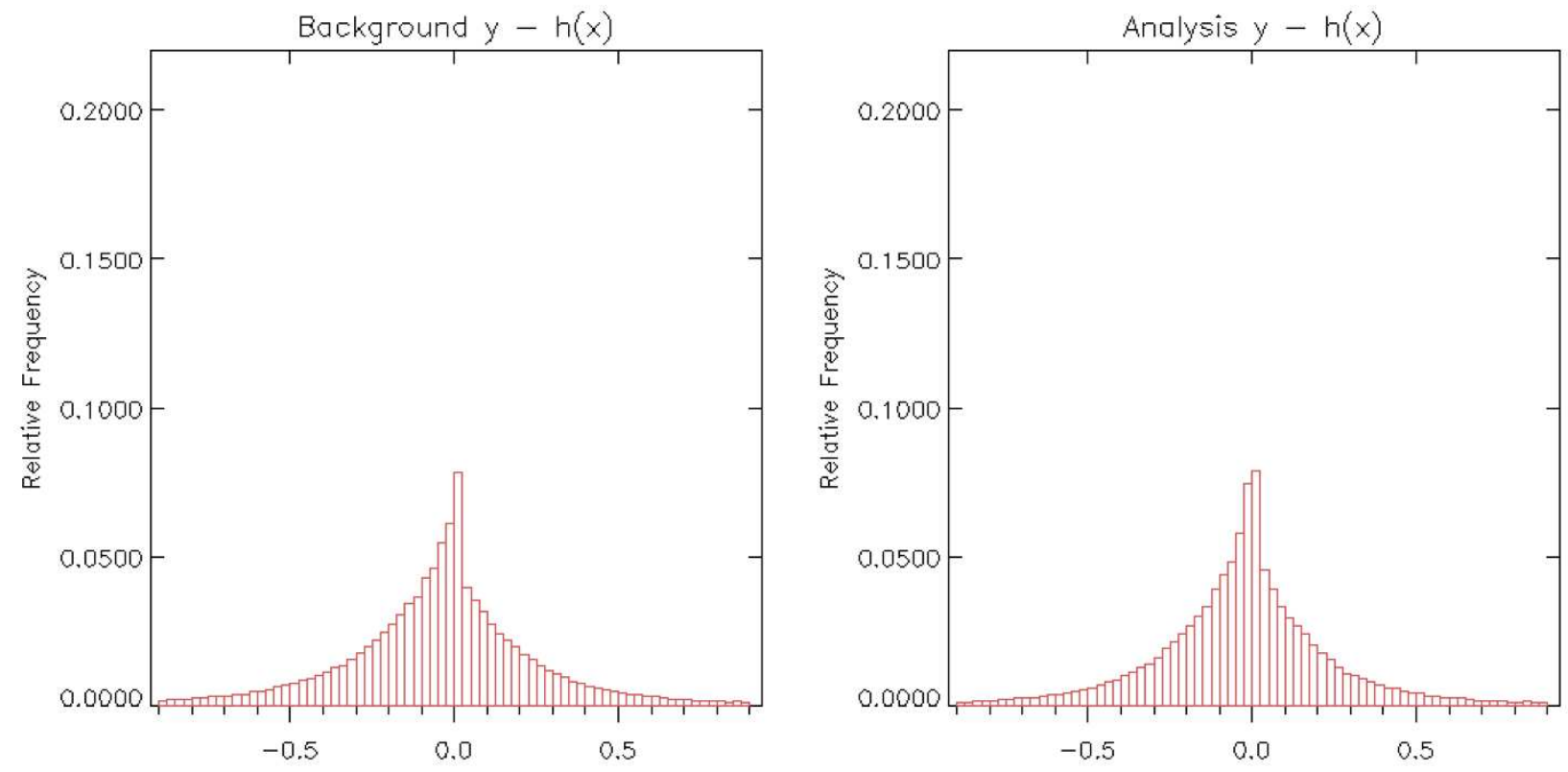

Figure 27 Histograms of observation-background for VV before (left) and after (right) DA simulation 

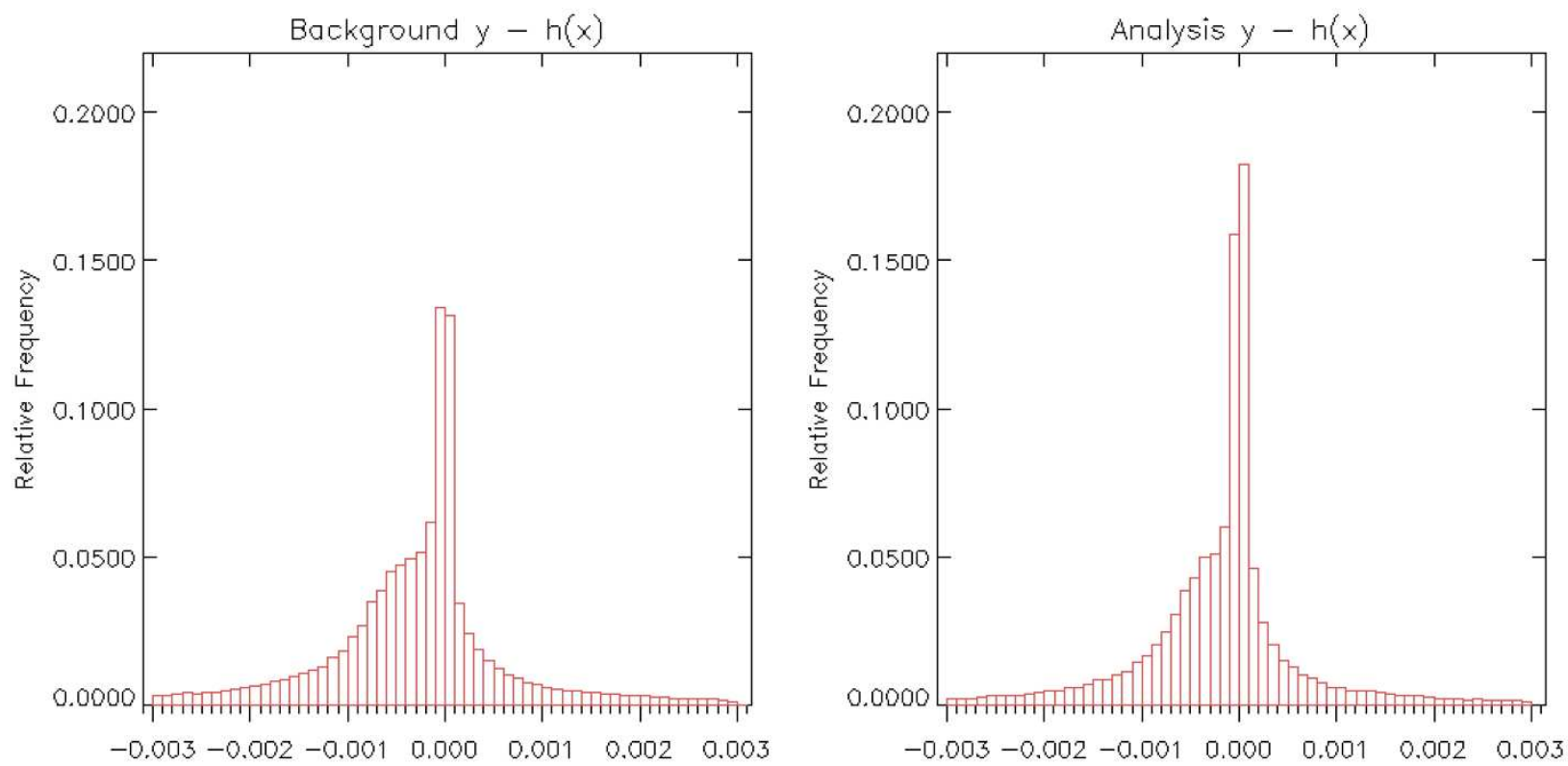

Figure 28 Histograms of observation-background for LH before (left) and after (right) DA simulation
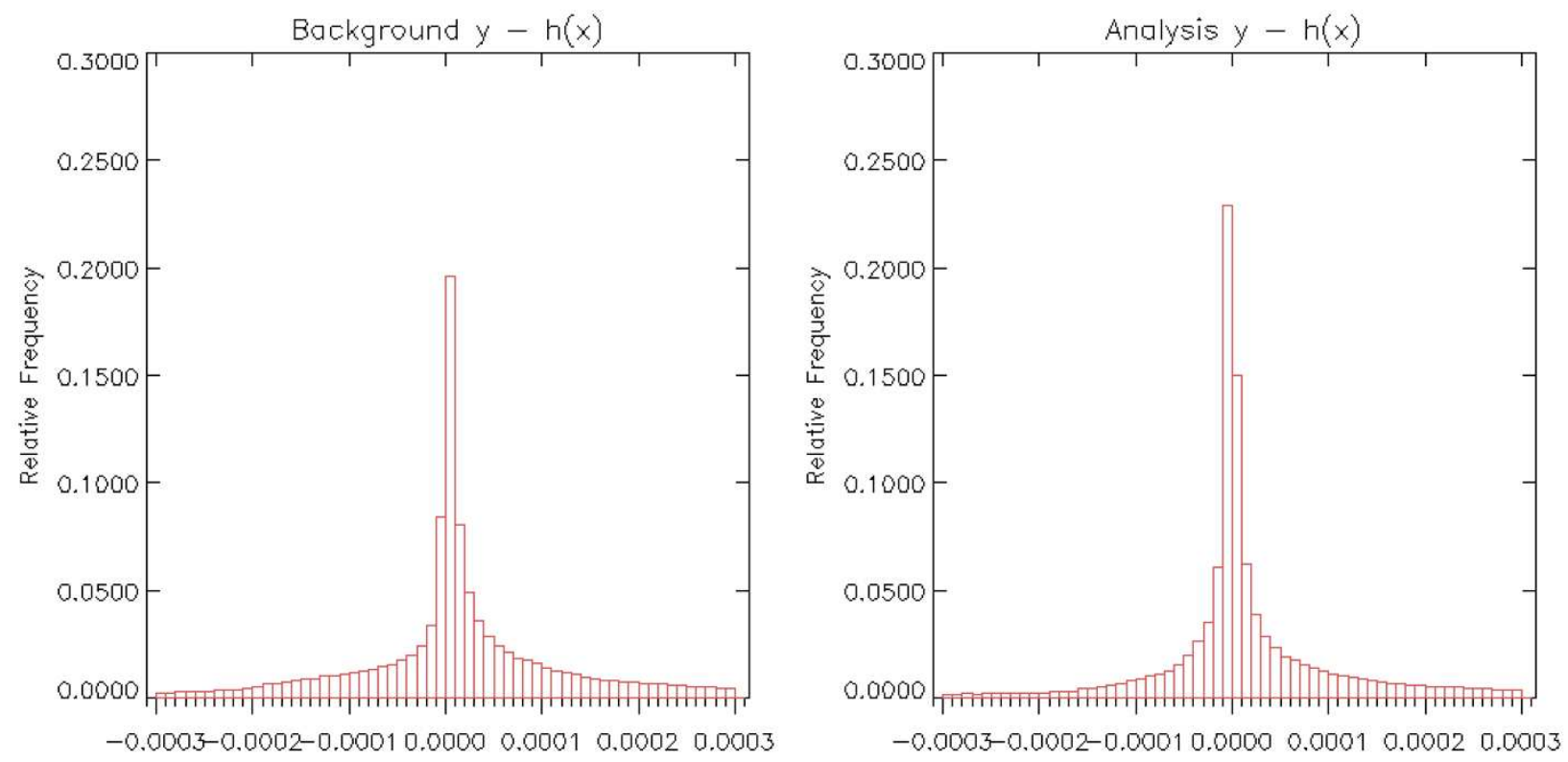

Figure 29 Histograms of observation-background for HYDRO before (left) and after (right) DA simulation 

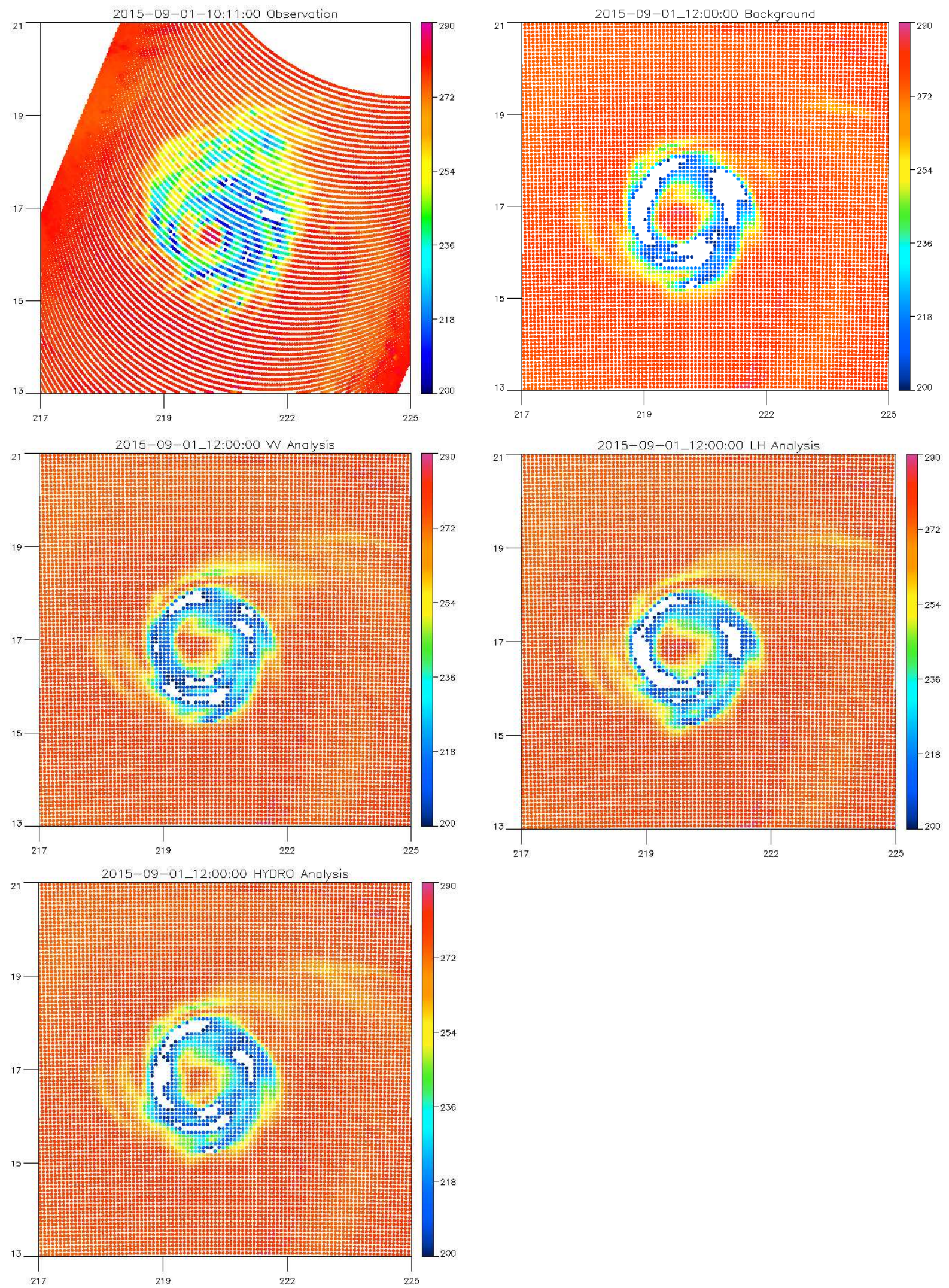

Figure 30 Brightness temperature map at $89 \mathrm{GHz} \mathrm{V}$ for Hurricane Jimena 

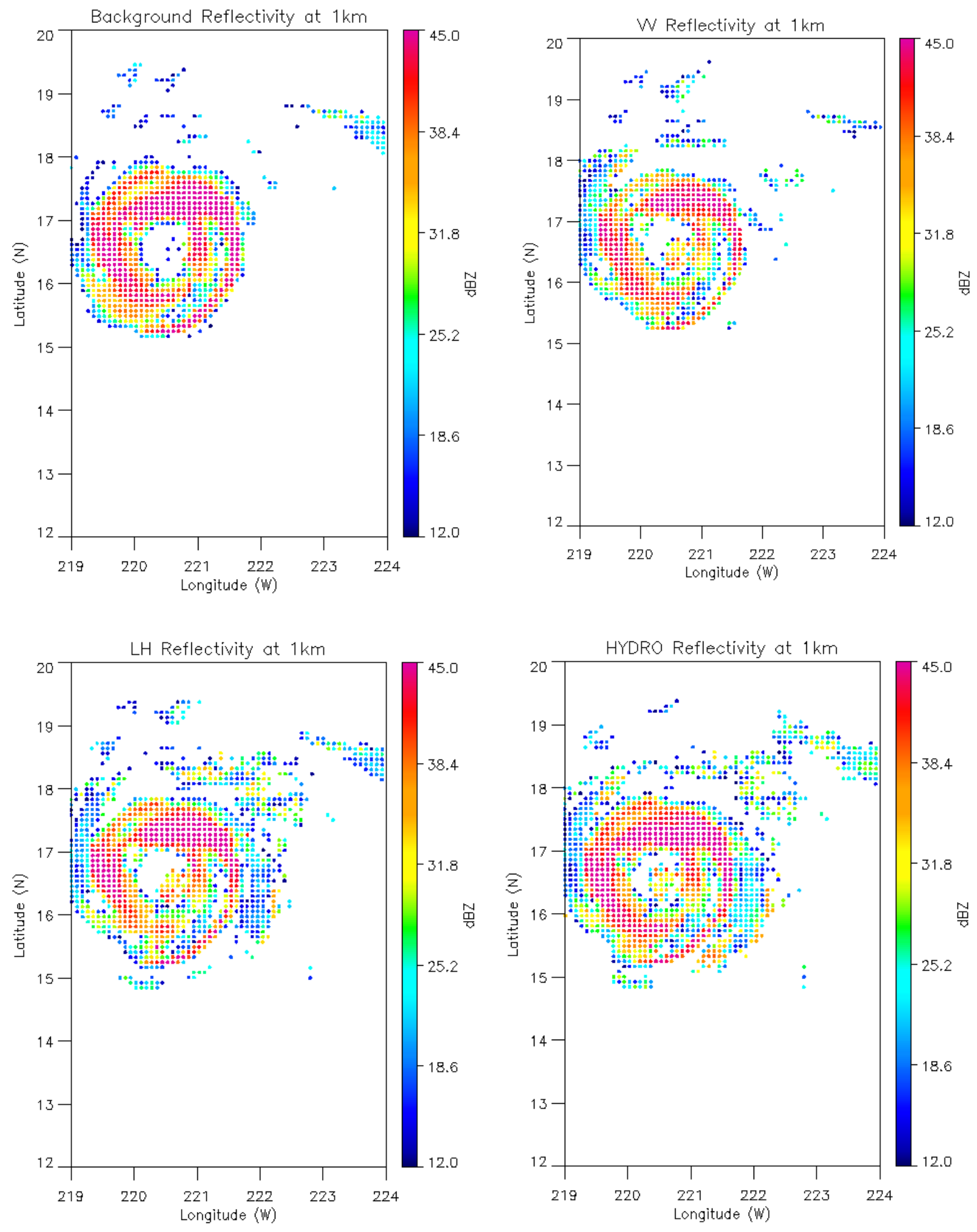

Figure 31 Horizontal cross-section of reflectivity at $1 \mathrm{~km}$ for Background, VV, LH, and HYDRO 

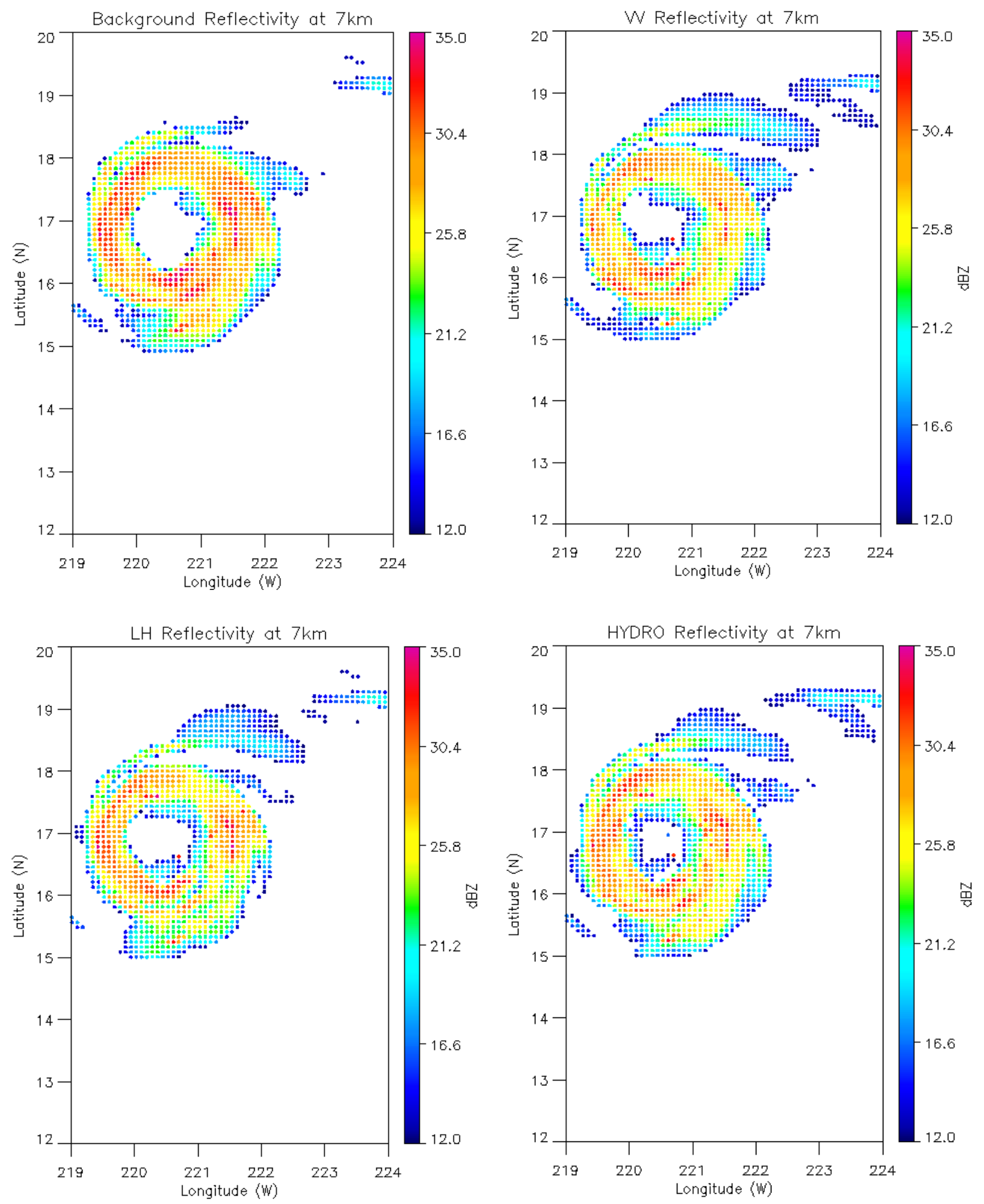

Figure 32 Horizontal cross-section of reflectivity at $7 \mathrm{~km}$ for Background, VV, LH, and HYDRO 

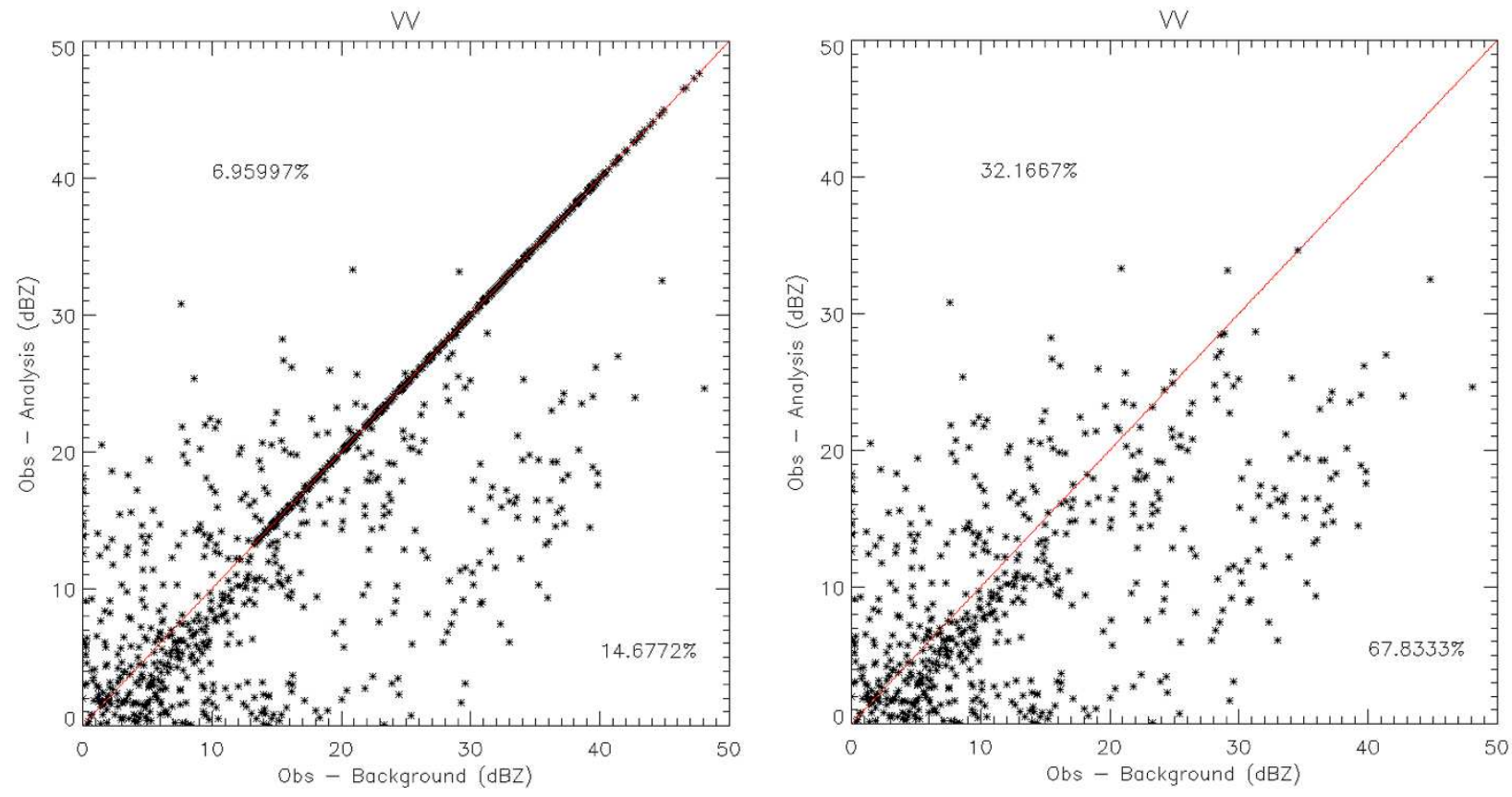

Figure 33 Scatter plot of difference between observation and analysis against difference between observation and background for VV including (left) and excluding(right) points that did not change
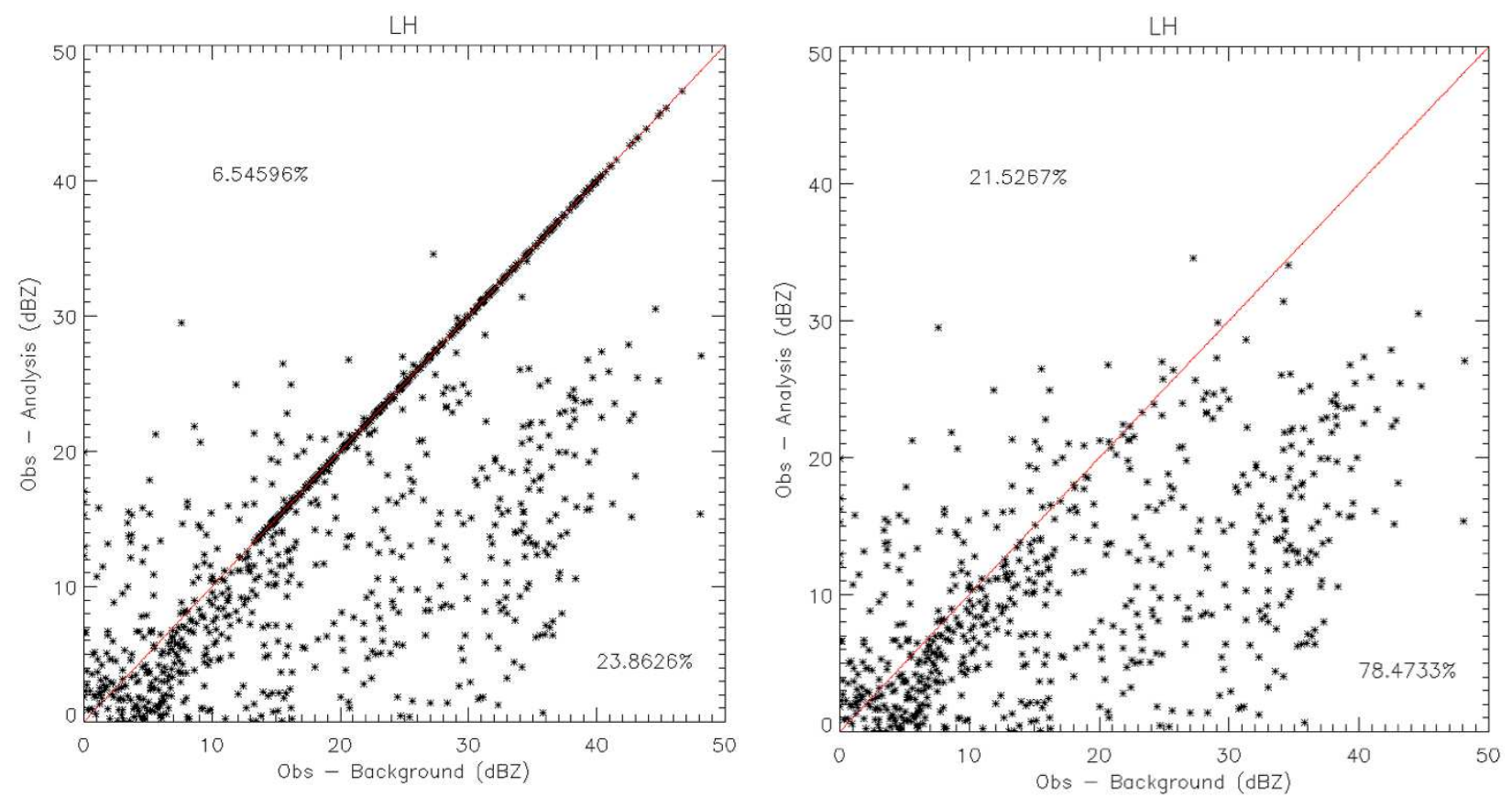

Figure 34 Scatter plot of difference between observation and analysis against difference between observation and background for LH including (left) and excluding (right) points that did not change 

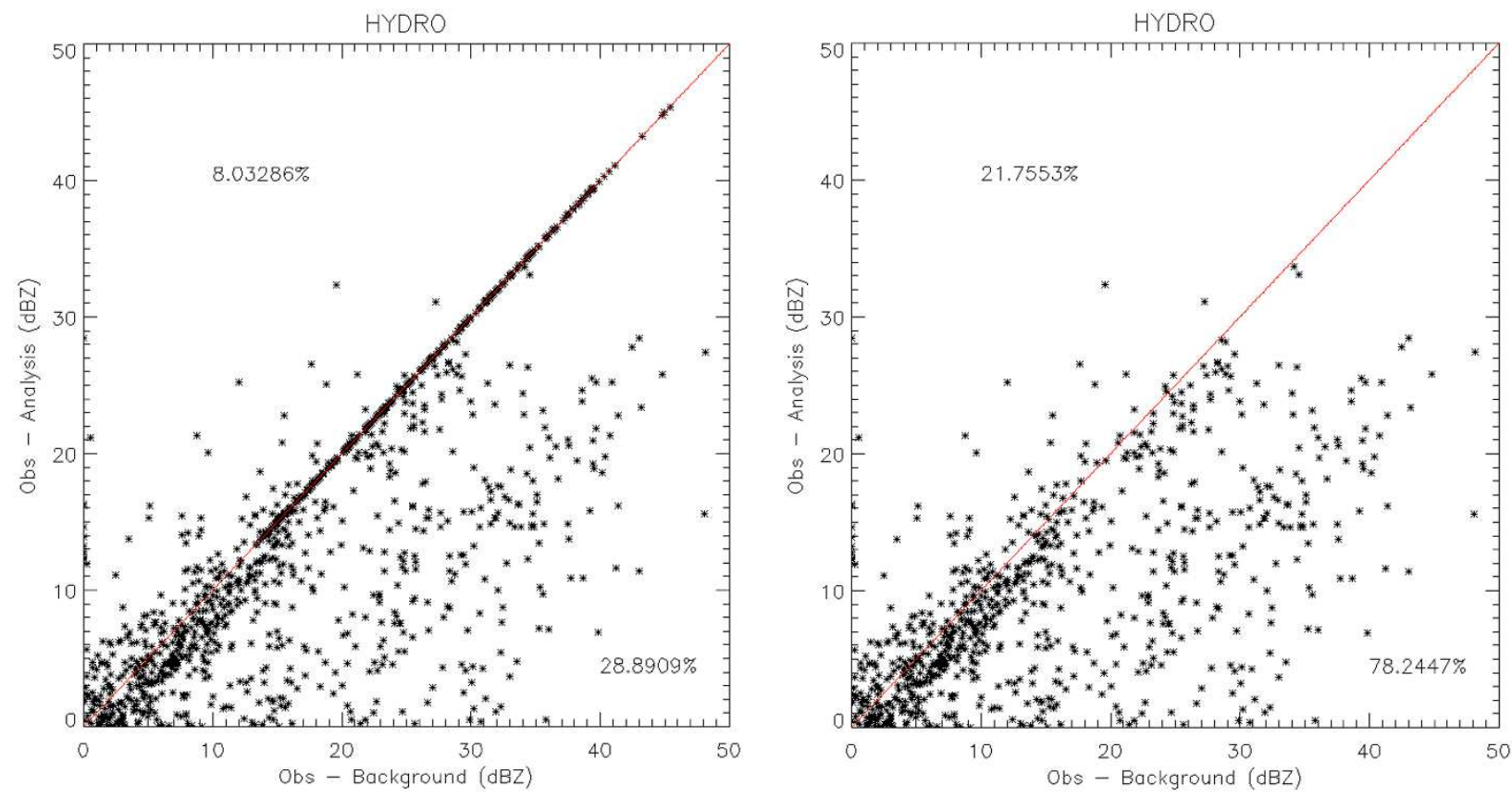

Figure 35 Scatter plot of difference between observation and analysis against difference between observation and background for HYDRO including (left) and excluding points that did not change

In addition to evaluating the analysis result, one more experiment was conducted with Hurricane Jimena to evaluate if the track forecast showed any improvement. The analysis itself was improved most by assimilating LH or HYDRO but, which experiment would predict a more accurate hurricane track if the analysis is given as an initial condition to the model? The hurricane track for each experiment was plotted in Figure 36 every 6 hours along with the observed track data. Best track data was obtained from ATCF and the track of the forecast was determined by a minimum sea level pressure which is a good indicator for a location of the TC core. All experiments, including the control run, started from the same location but began to diverge after that. LH had the least error after 6 hours, but later it was HYDRO that approached closer to the best track. LH and HYDRO take turns at better forecasting the track. 


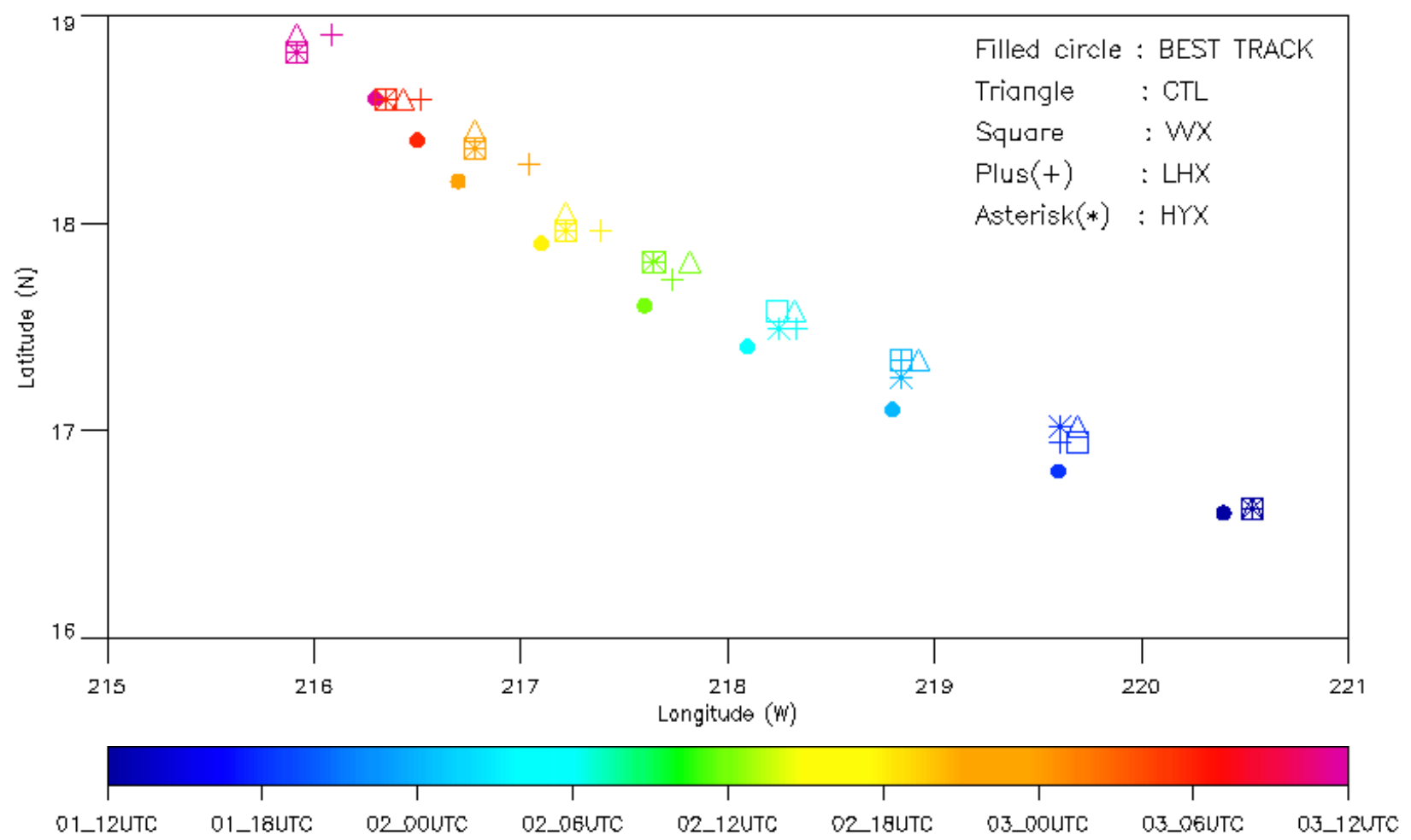

Figure 36 Hurricane Jimena track forecast for control, VV, LH, and HYDRO run 


\section{CHAPTER 5: DISCUSSION}

Three experiments using VV, LH, and HYDRO were conducted to compare the impact of using different variables on a data assimilation system, although all variables were derived from the same radar observations. The key factor that controls the result of the assimilation experiments in this study should be the observation error. The forecast error is given by an ensemble forecast while the observation error is provided by the user depending on observation types and the algorithm used. It ultimately determines how much observation data is allowed in the system. Figure 37 shows $\mathrm{T}_{\mathrm{b}}$ map of HYDRO in hurricane Pali's case if a different observation error had been chosen. In this additional DA experiment, the observation error was decreased by $50 \%$ or $0.00005 \mathrm{~kg} / \mathrm{kg}$. By decreasing the observation error, the system assigns more weight to the assimilated observation than the background data, but assimilates fewer observations because it only allows observations that are less different from the model background under the same QC $\left(\frac{|y-H(x)|}{\sqrt{R}}<3\right)$ constraint. In Figure 37, the upper rainband, where the difference between the observations and the backgrounds was quite large, is less improved than the previous HYDRO run because most of the observations in that region did not pass the QC. At a minimum, this shows the importance of properly estimating uncertainty in retrieved fields. 


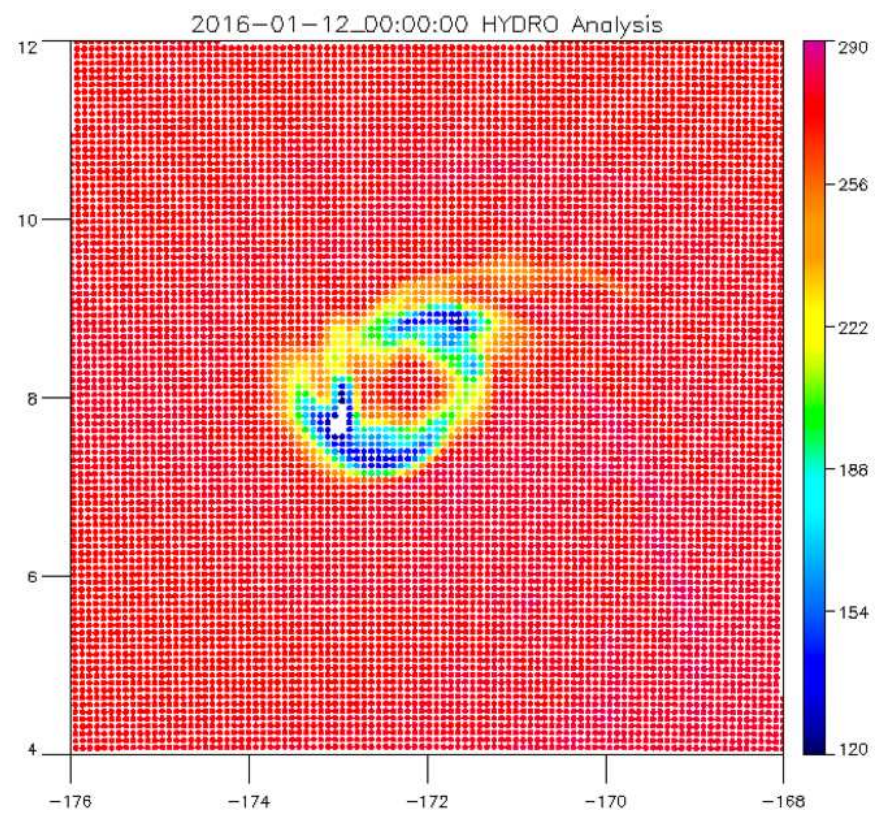

Figure 37 HYDRO run with a decreased observation error by half

There are also several points in this research that can be improved related to the observation operator. The assumption related to vertical grids of VV were made for simplicity, but it might have been more accurate to assimilate VV at its vertically staggered grid. In the case of LH, it was set as a control variable without taking additional covariance into account. It might have had a direct impact on the control variables if the equations to calculate the LH in the WDM6 can be used in the observation operator. However, the equations are spread out throughout the WDM6 scheme using a different time step depending on types of hydrometeors, making it difficult to incorporate in the observation operator where the different time step is not usually considered. Also in the WDM6 scheme, the equations contain too many variables (e.g. production rates of hydrometeors by various types of phase change) that should be added as control variables if the WDM6 scheme is used in the observation operator. This might be 
improved if there is a simple empirical equation with several related variables that can represent the microphysical scheme well enough.

Overall, LH and HYDRO runs surpassed VV. This may be due to a less linkage of a vertical motion in the microphysical scheme. HYDRO seems to be a little superior than LH but it is hard to conclude which variable is better. Similar results from LH and HYDRO in brightness temperature and reflectivity suggests that the two variables have the similar impact to the DA system. This could have been anticipated because LH and HYDRO are closely related to each other. The only thing that makes these two experiments different would be the computational time. As mentioned in Chapter 4, the number of observation for HYDRO was almost twice that for LH and this makes HYDRO be computationally more expensive than LH. In this sense, LH might be a better option if one wants to have a similar effect of assimilating HYDRO but time is a critical factor. 


\section{CHAPTER 6: SUMMARY AND CONCLUSION}

Drop size distributions are a critical factor in predicting radiances and reflectivities. Because models and retrievals often assume different DSD, it is hard to make them consistent with each other. This leads to an inconsistency problem when observation data are assimilated into the forecast model through an observation operator with a different DSD or when products that were retrieved from observations with different DSDs are assimilated into the model.

Despite the difficulty in matching DSDs between the observation and the model, the attempt to assimilate observations over cloudy regions continue to increase because there is valuable information about the scene. Observation data can be assimilated in many different ways with various variables, but it is not easy to find an optimal DA method and variables that can be assimilated to transfer information as effectively as possible.

In this study, a method that maintains consistency in DSD between observation and forecast model is presented. Consistency has been ensured by using WRF model outputs to build a retrieval framework. Radar observations are translated into retrieved parameters by searching a database of model fields for which the appropriate reflectivity profile has been computed. With this method, each of three variables (vertical velocity, latent heating, and hydrometeor water contents) were retrieved, and assimilated to determine which variable has the most positive impact. Each DA experiment was conducted with two TC cases. LH and HYDRO had the most improvement in the analysis of each $\mathrm{TC}$, but their results were comparable to each other in both cases. However, LH can be considered to be superior to HYDRO in terms of computational time because LH and HYDRO are so closely related to each other that it appears that the same 
information distributed to five types of hydrometeors is compressed into one variable of latent heating. Both LH and HYDRO showed improvements in TC track forecast of one TC. 


\section{REFERENCES}

Andersson, E., J. Pailleux, J.R. Eyre, A.P. McNally, G.A. Kelly, P. Courtier, and F. Rabier, 1993: Assimilation of satellite data by 3D-Var at ECMWF. Proc. ECMWF Seminar on Developments in the Use of Satellite Data in Numerical Wea. Prediction. 1993, Reading, England, ECMWF, 167-188. [Available online at http://www.ecmwf.int/sites/default/files/elibrary/1993/7742-assimilation-satellite-data$\underline{\text { 3d-var-ecmwf.pdf] }}$

Benjamin, S. G., and Coauthors, 2016: A North American hourly assimilation and model forecast cycle: The Rapid Refresh. Mon. Wea. Rev., 144, 1669-1694, doi:10.1175/MWR-D-15-0242.1.

Bormann, A., A. Geer, and S. English, 2012: Evaluation of the microwave ocean surface emissivity model FASTEM-5 in the IFS. ECMWF Technical memorandum 667, ECMWF, 18pp

Caplan, P., J. Derber, W. Gemmill, S.-Y. Hong, H.-L. Pan, and D. Parrish, 1997: Changes to the 1995 NCEP operational Medium-Range Forecast Model Analysis-Forecast System. Wea. Forecasting, 12, 581-594, doi:10.1175/1520-0434(1997)012,0581: CTTNOM.2.0.CO;2.

Derber, J. C., and W.-S. Wu, 1998: The use of TOVS cloud-cleared radiances in the NCEP SSI analysis system. Mon. Wea. Rev., 126, 2287-2299, doi:10.1175/15200493(1998)126,2287: TUOTCC.2.0.CO;2.

Gallus, W. A., Jr., and M. Pfeifer, 2008: Intercomparison of simulations using 5 WRF microphysical schemes with dual-polarization data for a German squall line. $A d v$. Geosci., 16, 109-116.

Han, M., S. A. Braun, T. Matsui, and C. R. Williams, 2013: Evaluation of cloud microphysics schemes in simulations of a winter storm using radar and radiometer measurements. $J$. Geophys. Res. Atmos., 118, 1401-1419, doi:10.1002/ jgrd.50115.

Hou, A. Y., and Coauthors, 2014: The Global Precipitation Measurement mission. Bull. Amer. Meteor. Soc., 95, 701-722, doi:10.1175/BAMS-D-13-00164.1.

Kalnay, E., 2003: Atmospheric Modeling, Data Assimilation, and Predictability. Cambridge University Press, $341 \mathrm{pp}$

Knapp, K. R., M. C. Kruk, D. H. Levinson, H. J. Diamond, and C. J. Neumann, 2010: The International Best Track Archive for Climate Stewardship (IBTrACS): Unifying tropical cyclone data. Bull. Amer. Meteor. Soc., 91, 363-376, doi:10.1175/ 2009BAMS2755.1. Koller, D., and N. Friedma 
Kummerow, C., 1993: On the accuracy of the Eddington approximation for radiative transfer in the microwave frequencies. J. Geophys. Res., 98, 2757-2765.

Lim, K.-S. S., and S.-Y. Hong, 2010: Development of an effective double-moment cloud microphysics scheme with prognostic cloud condensation nuclei (CCN) for weather and climate models. Mon. Wea. Rev., 138, 1587-1612, doi:10.1175/2009MWR2968.1.

Liu, H., and J. Li, 2010: An improvement in forecasting rapid intensification of Typhoon Sinlaku (2008) using clear-sky full spatial resolution advanced IR soundings. J. Appl. Meteor. Climatol., 49, 821-827.

Matricardi, M., F. Chevallier, and S. Tjemkes, 2001: An improved general fast radiative transfer model for the assimilation of radiance observations. ECMWF Research Dept. Tech. Memo. 345, $42 \mathrm{pp}$

Okamoto, K., K. Aonashi, T. Kubota, T. Tashima, 2016: Experimental Assimilation of the GPM Core Observatory DPR Reflectivity Profiles for Typhoon Halong (2014). Mon. Wea. Rev., 144, 2307-2326, doi:10.1175/MWR-D-15-0399.1

Petty, G. W., and W. Huang, 2011: The modified gamma size distribution applied to inhomogeneous and nonspherical particles: Key relationships and conversions. J. Atmos. Sci., 68, 1460-1473, doi:10.1175/2011JAS3645.1.

Skamarock, W. C., and coauthors, 2008: A description of the Advanced Research WRF version 3. NCAR Tech. Note NCAR/TN-475+STR, $113 \mathrm{pp}$.

Xu, D., Z. Liu, X.-Y. Huang, J. Min, and H. Wang, 2013: Impact of assimilating IASI radiance observations on forecasts of two tropical cyclones. Meteor. Atmos. Phys., 122, 1-18, doi:10.1007/s00703-013-0276-2.

Zhang, M., M. Zupanski, M.-J. Kim, and J. A. Knaff, 2013: Assimilating AMSU-A radiances in the TC core area with NOAA operational HWRF (2011) and a hybrid data assimilation system: Danielle (2010). Mon. Wea. Rev., 141, 3889- 3907, doi:10.1175/MWR-D-1200340.1 .

Zupanski, M., 2005: Maximum likelihood ensemble filter: Theoretical aspects. Mon. Wea. Rev., 133, 1710-1726, doi:10.1175/ MWR2946.1.

Figure 2:

GPM satellite images for Hurricane Pali [online image]

from https://pmm.nasa.gov/extreme-weather/gpm-spots-hurricane-pali-forming

5 day track for Hurricane Pali [online image]

from http://www.prh.noaa.gov/cphc/tc_graphics/latest_w.php?stormid=CP012016

Figure 3: 
GPM satellite images for Hurricane Jimena [online image]

from https://phys.org/news/2015-09-gpm-hurricane-jimena-intense-eyewall.html

5 day track for Hurricane Jimena [online image]

from http://www.prh.noaa.gov/cphc/tc_graphics/latest_w.php?stormid=EP132015 


\section{APPENDIX A}

Brightness temperature and reflectivity are sensitive to the parameters of the DSD. In the RTM, optical properties of hydrometeors are calculated based on the assumed DSD and their impacts on the brightness temperature and the reflectivity can be significant. The gamma size distribution is one of the most common forms of DSD and its normalized form can be expressed as follows.

$$
\begin{gathered}
N(D)=N_{w} f(\mu)\left(\frac{D}{D_{0}}\right)^{\mu} \exp \left[-(3.67+\mu) \frac{D}{D_{0}}\right] \\
\text { where } N_{w}=\frac{3.67^{4}}{\pi \rho_{w}}\left(\frac{10^{3} W}{D_{0}^{4}}\right)\left(\mathrm{mm}^{-1} \mathrm{~m}^{-1}\right) \text { and } f(\mu)=\frac{6}{3.67^{4}} \frac{(3.67+\mu)^{4}}{\Gamma(\mu+4)}
\end{gathered}
$$

The normalized intercept parameter $\left(\mathrm{N}_{\mathrm{w}}\right)$, median volume diameter $\left(\mathrm{D}_{0}\right)$, and shape parameter ( $\mu$ ) are the main parameters that define the distribution. $W$ is water content $\left(\mathrm{g} / \mathrm{m}^{3}\right), \rho_{\mathrm{w}}$ is the density of the hydrometeor and $\Gamma$ is the gamma function $(\Gamma(x+1)=x !)$.

In this appendix, each of the three DSD parameters were varied in the RTM to examine their impacts on brightness temperature and reflectivity of precipitating hydrometeors (rain, snow, and graupel). Cloud liquid water and cloud ice are defined as small particles of the precipitating hydrometeors that have the same optical properties in the RTM. Figures A1, A2, and $\mathrm{A} 3$ shows changes in the $\mathrm{T}_{\mathrm{b}} \mathrm{s}$ for the distinct GMI frequencies when water content and $\mathrm{D}_{0}$ are varied for rain, snow, and graupel respectively. $\mathrm{D}_{0}$ was varied from 0.05 to $5 \mathrm{~mm}$ while black, blue, and red lines respectively represent $0.1 \mathrm{~g} / \mathrm{m}^{3}, 0.5 \mathrm{~g} / \mathrm{m}^{3}$, and $2.5 \mathrm{~g} / \mathrm{m}^{3}$ of total water content. Increasing water content directly affects the magnitude of both absorption and scattering because the number of drops is proportional to the water content. Rain (liquid water) has a relatively large imaginary part of the refractive index which is related to the absorption efficiency and this 
makes the absorption dominate over scattering at lower frequency (10.65 and 18.7GHz). $\mathrm{T}_{\mathrm{b}}$ increases slowly as the diameter increases because the absorption by raindrops increases as the relative size grows towards the Mie regime. Then $T_{b}$ starts to decrease because scattering comes into play by reflecting some of the radiation coming from lower layers back toward the surface. $\mathrm{T}_{\mathrm{b}}$ does not vary much at $23.8 \mathrm{GHz}$ because this channel is sensitive to water vapor. At the higher frequency $(89 \mathrm{GHz}), \mathrm{T}_{\mathrm{b}}$ decreases by scattering but starts to increase as the diameter increases due to the increased amount of forward scattering. At $166 \mathrm{GHz}$, it follows a similar pattern as $89 \mathrm{GHz}$ but does not vary much because the channel is close to a strong water vapor absorption band. Finally, around $183 \mathrm{GHz}, \mathrm{T}_{\mathrm{b}}$ stays the same due to a saturation achieved by water vapor absorption. In the case of ice hydrometeors, their imaginary part of the refractive index is small and the absorption is almost negligible. Scattering is also small at low frequencies, leading to negligible $T_{b}$ differences with diameter, but at $89.0 \mathrm{GHz}$, scattering increases and $T_{b}$ become quite sensitive to the diameter. Channels behave similarly at a strong water vapor absorption channels (166 and $183 \mathrm{GHz}$ ) because the absorbed radiation by water vapor is scattered by ice hydrometeors that are located above where the weighting function peaks in water vapor.

Compared to the water contents and the $\mathrm{D}_{0}, \mathrm{~T}_{\mathrm{b}}$ is less sensitive to $\mu$ for all hydrometeors. This is shown in Figure A4, A5, and A6. 

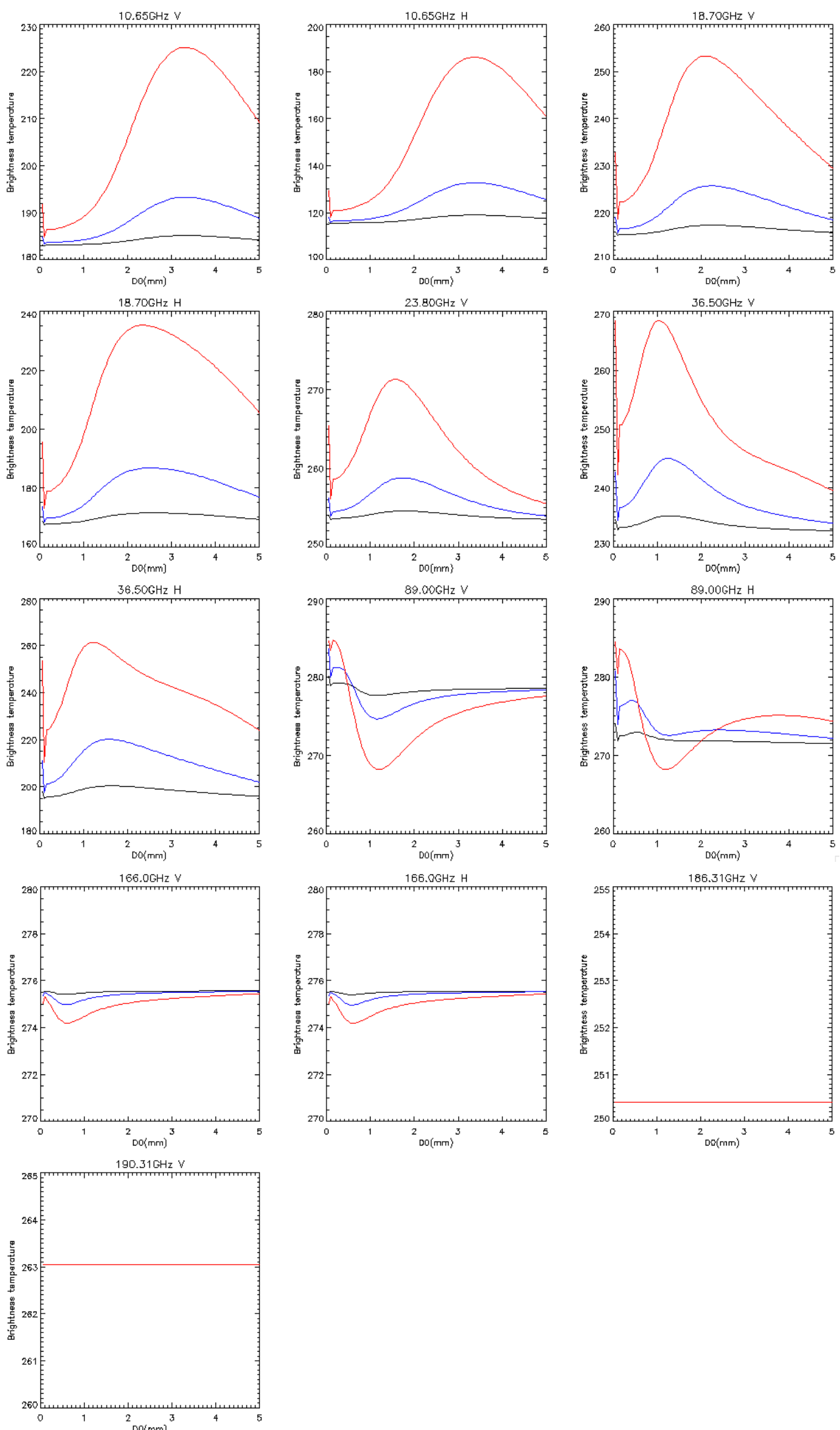

Figure A1 Plots of brightness temperature for rain varied by water content (Black, blue, and red for $0.1 \mathrm{~kg} / \mathrm{m} 3,0.5 \mathrm{~kg} / \mathrm{m} 3$, and $2.5 \mathrm{~kg} / \mathrm{m} 3$ respectively) and the median volume diameter 

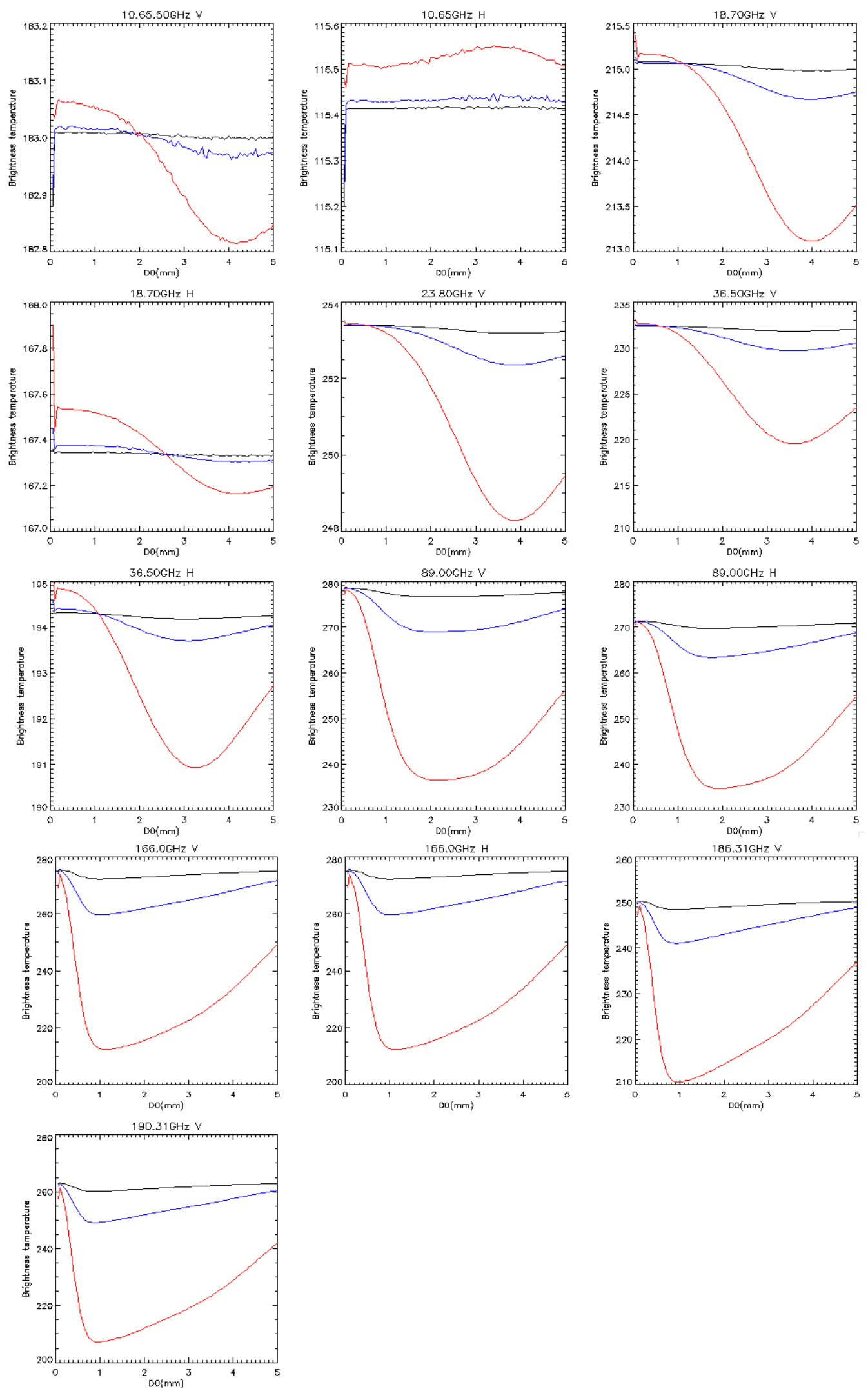

Figure A2 Plots of brightness temperature for snow varied by water content (Black, blue, and red for $0.1 \mathrm{~kg} / \mathrm{m} 3,0.5 \mathrm{~kg} / \mathrm{m} 3$, and $2.5 \mathrm{~kg} / \mathrm{m} 3$ respectively) and the median volume diameter 

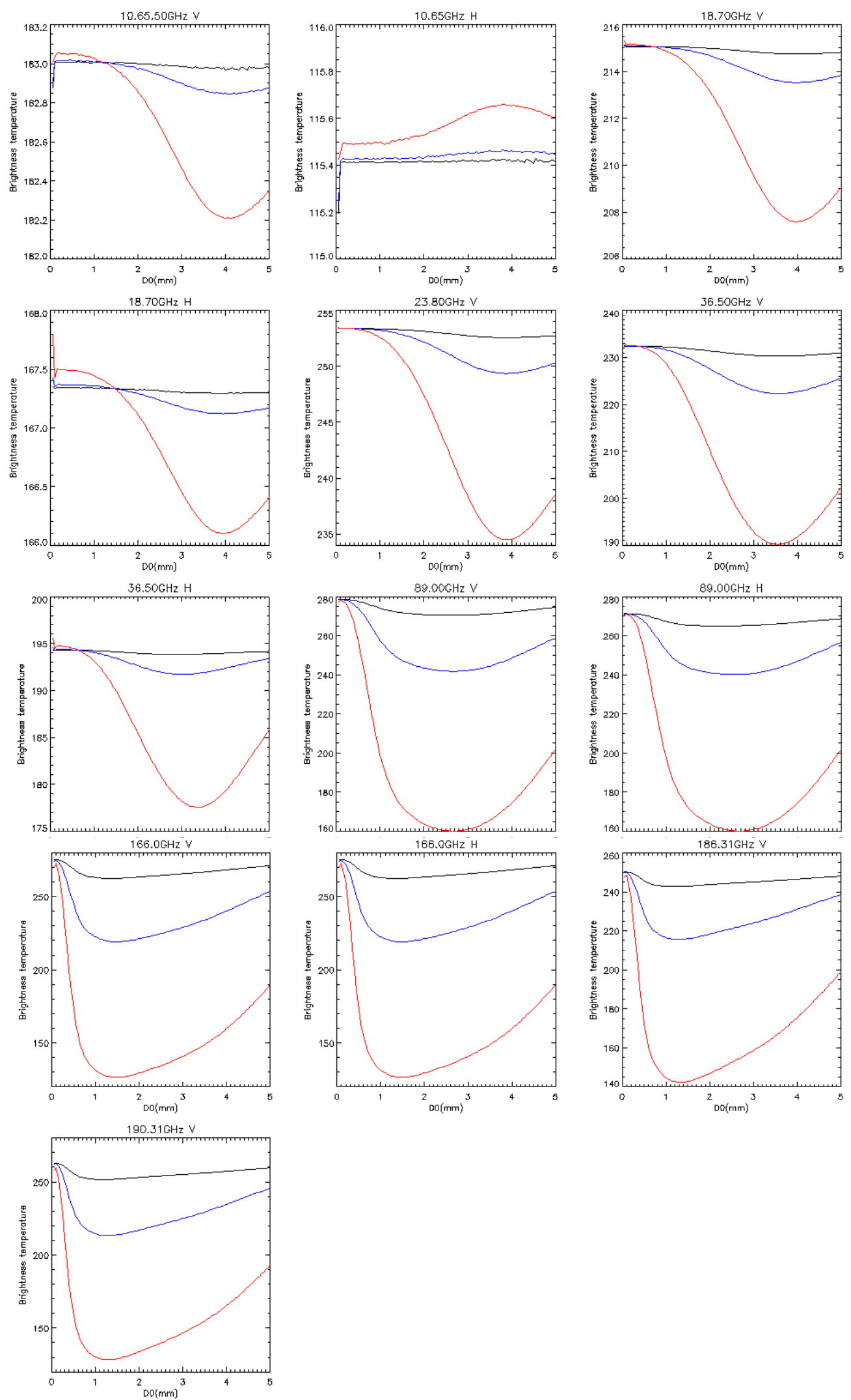

Figure A3 Plots of brightness temperature for graupel varied by water content (Black, blue, and red for $0.1 \mathrm{~kg} / \mathrm{m} 3,0.5 \mathrm{~kg} / \mathrm{m} 3$, and $2.5 \mathrm{~kg} / \mathrm{m} 3$ respectively) and the median volume diameter 

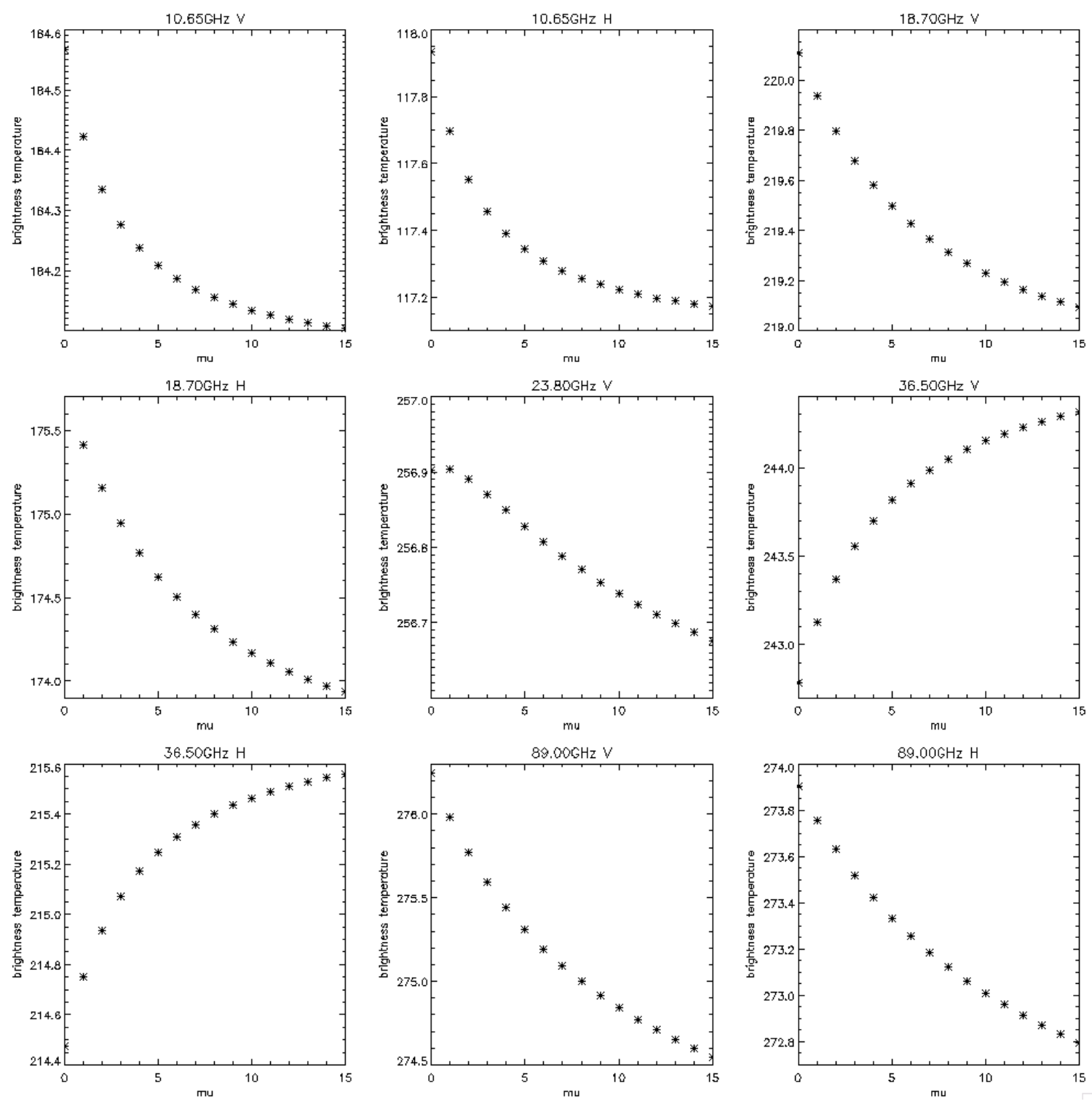

Figure A4 Plots of brightness temperature for rain varied by $\mu$ 

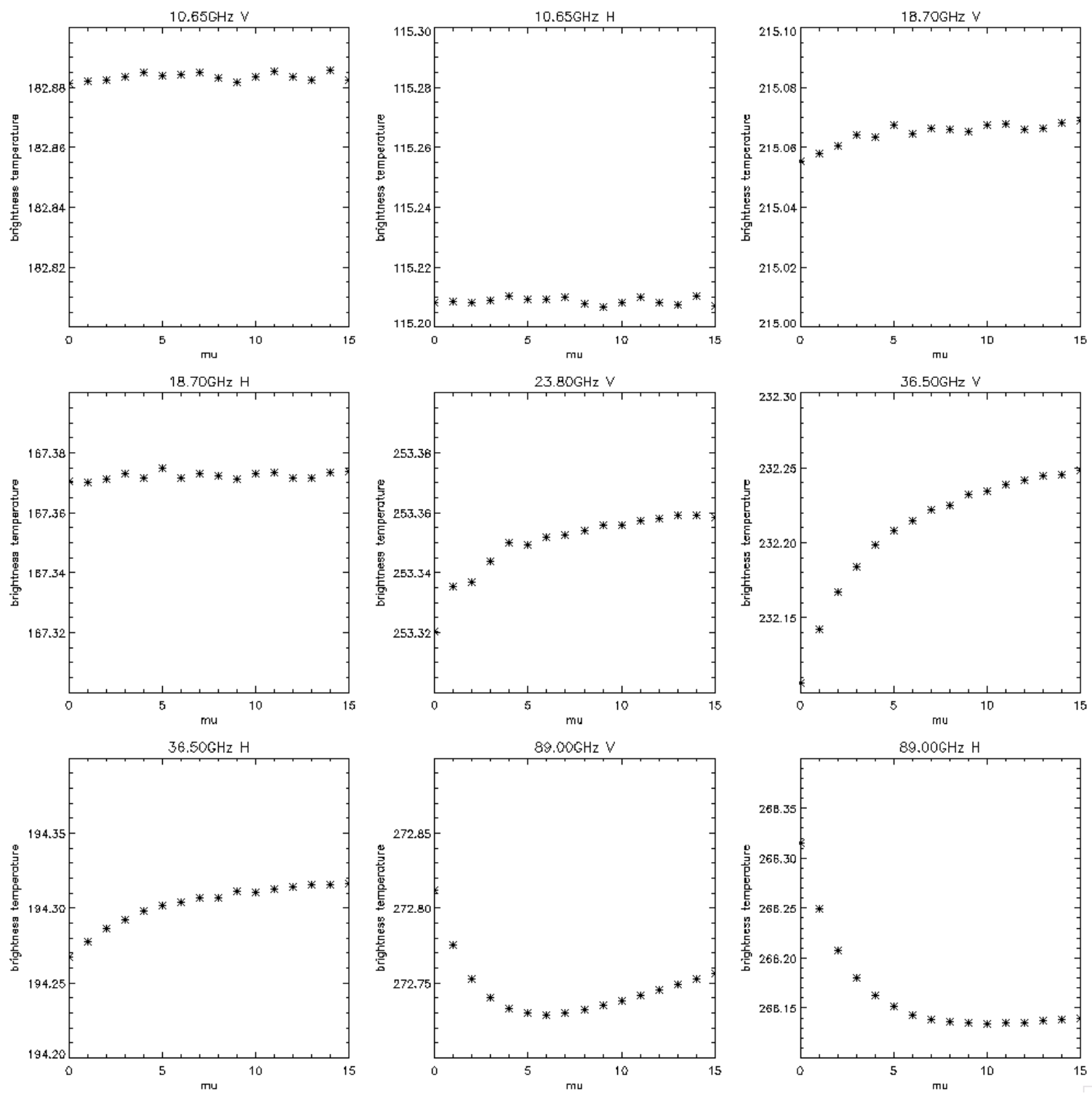

Figure A5 Plots of brightness temperature for snow varied by $\mu$ 

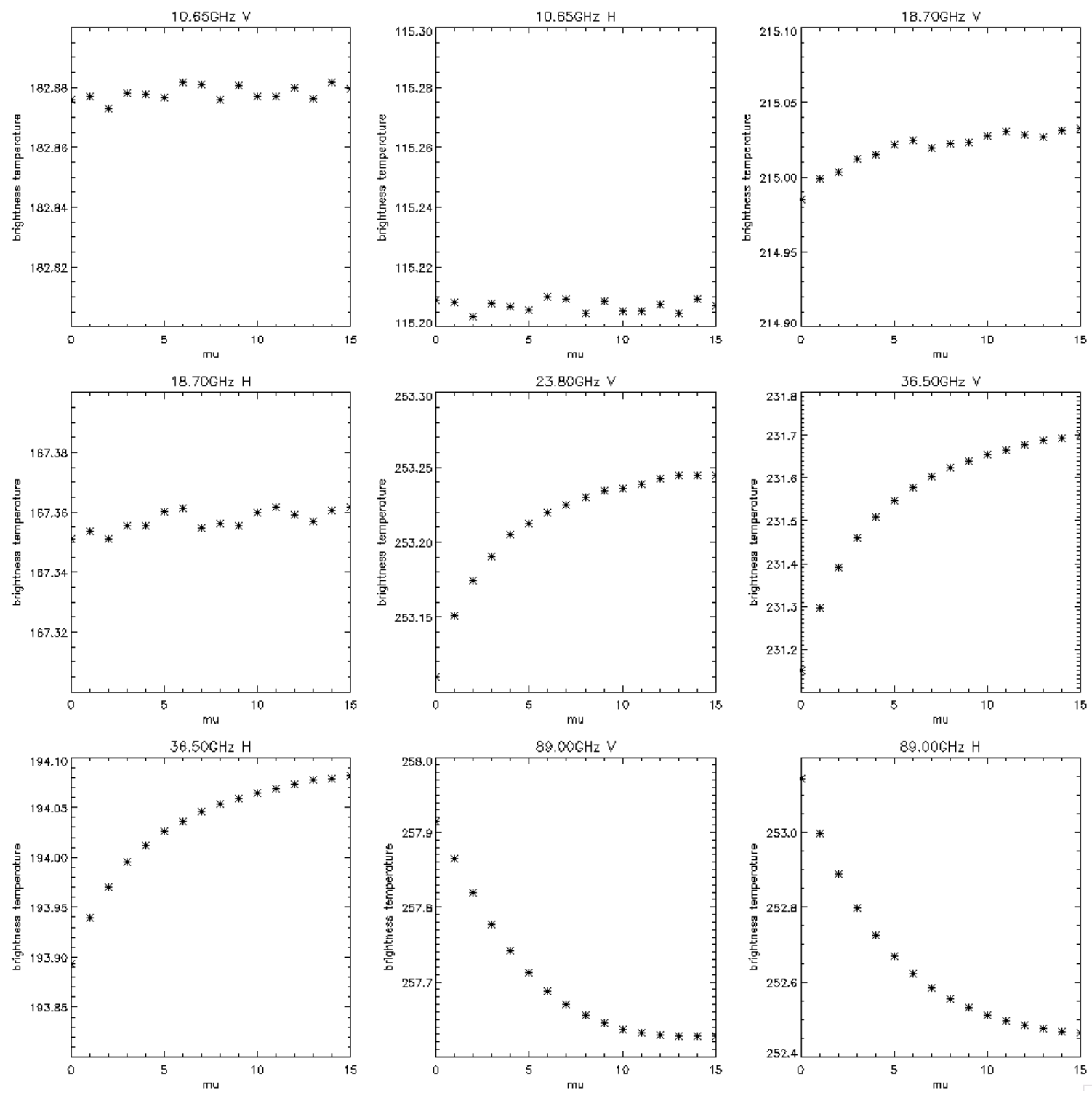

Figure A6 Plots of brightness temperature for graupel varied by $\mu$ 
The same experiments were conducted for reflectivity of rain, snow, and graupel. They were examined at $13.6 \mathrm{GHz}$ in $\mathrm{Ku}-\mathrm{band}$ and it is shown in Figure A7 and A8. The magnitude of the reflectivity for snow and graupel is relatively smaller than that for rain because rain backscatters more than ice hydrometeors at this frequency (due to the dielectric property of water). Reflectivity for graupel is a little bit greater than snow because it is denser. A bright band is caused by a large reflectivity of liquid water coating when it begins to coat large snow particles as they begin to melt. Varying $\mu$ does not affect reflectivity much for all the hydrometeors as in $T_{b}$. 

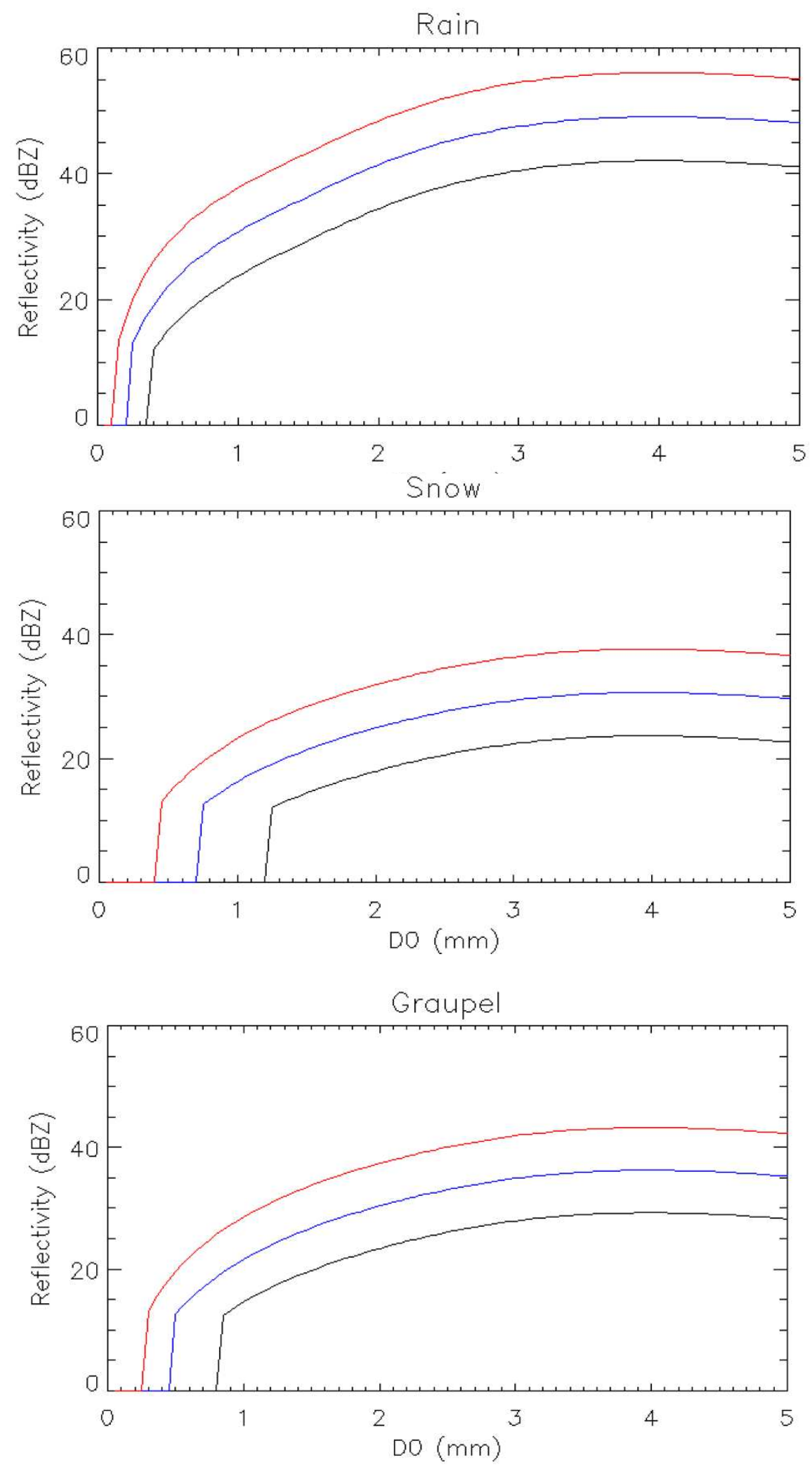

Figure A7 Plots of reflectivity for rain, snow, and graupel varied by water content (Black, blue, and red for $0.1 \mathrm{~kg} / \mathrm{m} 3,0.5 \mathrm{~kg} / \mathrm{m} 3$, and $2.5 \mathrm{~kg} / \mathrm{m} 3$ respectively) and the median volume diameter 

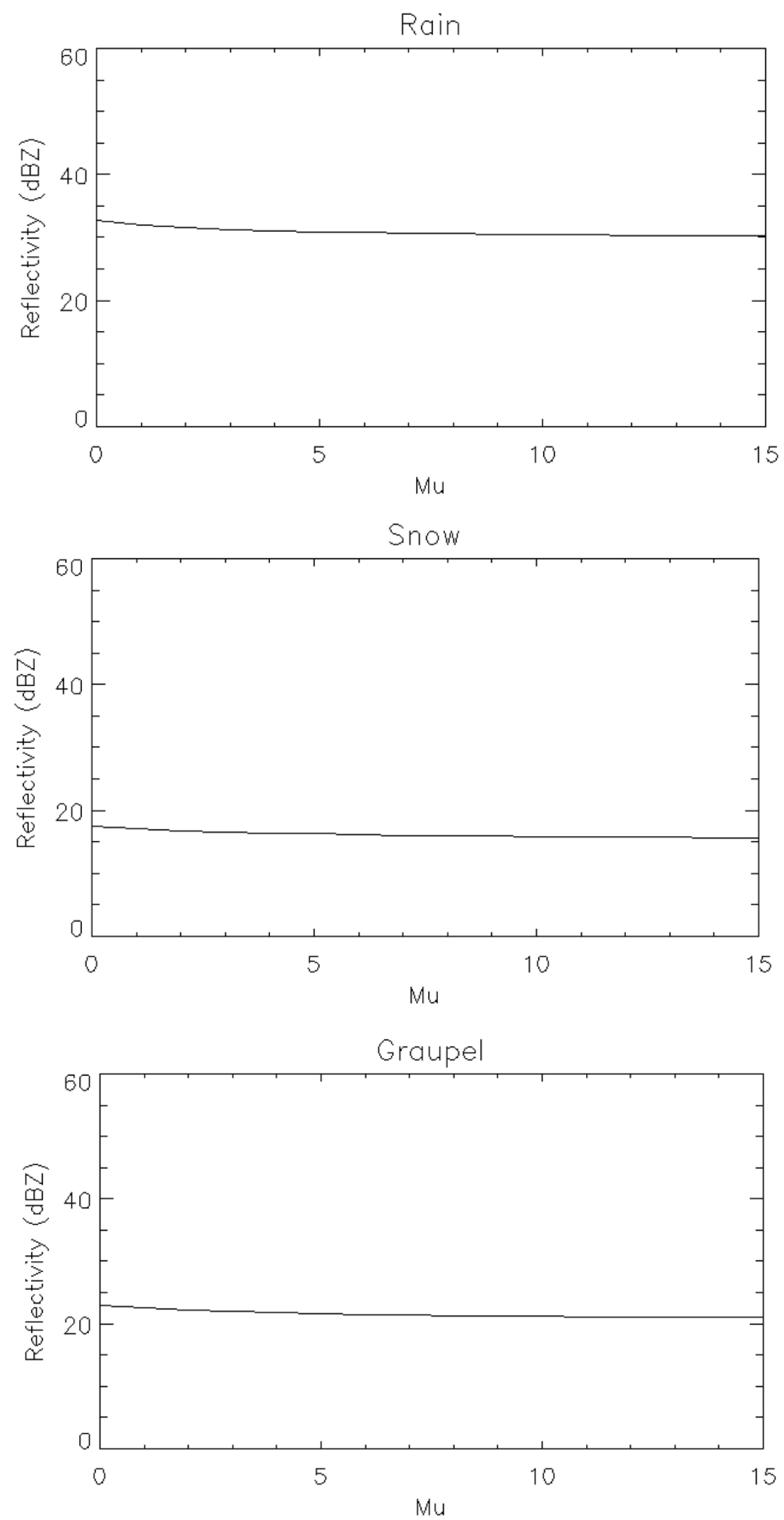

Figure A8 Plots of reflectivity for rain, snow, and graupel varied by $\mu$ 